\title{
Enantioselective $\alpha$-Arylation of Primary Alcohols under Sequential One-Pot Catalysis
}

\author{
Bruno Lainer, Dawid Lichosyt,* Maiia Aleksandrova, and Paweł Dydio* \\ University of Strasbourg, CNRS, ISIS UMR 7006, 8 allée Gaspard Monge, 67000 Strasbourg, France
}

This file includes:

I. Table S1 - Optimization of Reaction Conditions and Control Experiments .......................S2

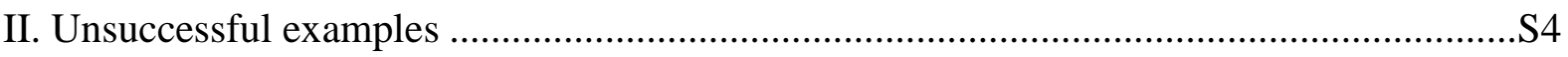

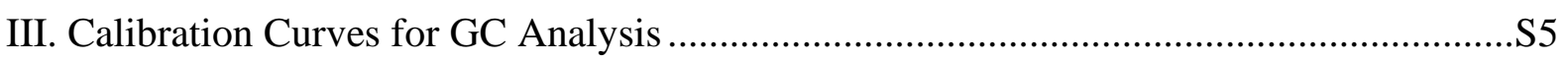

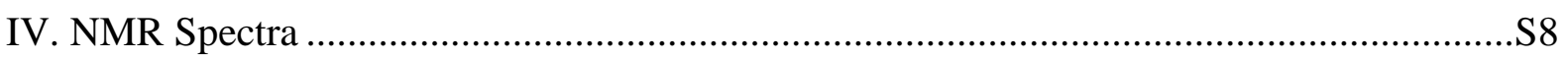

IV.A NMR Spectra of the Prepared Substrates ..........................................................S8

IV.B NMR Spectra of the Products ............................................................................

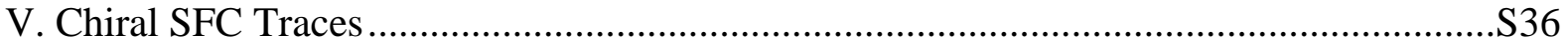

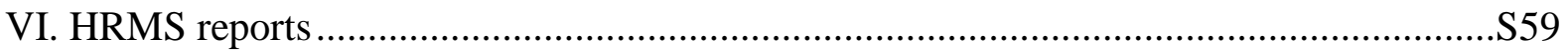




\section{Table S1 - Optimization of Reaction Conditions and Control Experiments}

1) $\left[\mathrm{RuCl}_{2} \text { (cymene) }\right]_{2}(2.5 \mathrm{~mol} \%)$,

rac-binap (5 mol\%), NaOAc (5 mol\%),

$\mathrm{N}$-ethyl maleimide ( 1.1 equiv.)

Toluene $(1 \mathrm{~mL}), 80^{\circ} \mathrm{C}, 5 \mathrm{~h}$

$\sim \mathrm{NH}^{\mathrm{O}}$

2) $\left[\mathrm{RuCl}_{2}(\text { cymene })\right]_{2}(2.5 \mathrm{~mol} \%)$,

$0.25 \mathrm{mmol}$

$(S, S)$-me-bipam (5.5 mol\%), $\mathrm{K}_{2} \mathrm{CO}_{3}$ (1 equiv.)

$\mathrm{PhB}(\mathrm{OH})_{2}$ (3 equiv.), $\mathrm{H}_{2} \mathrm{O}$ (150 uL),

Toluene $(2 \mathrm{~mL}), 60^{\circ} \mathrm{C}, 6 \mathrm{~h}$

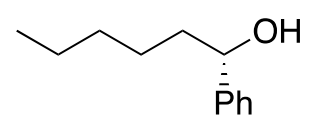

\begin{tabular}{|c|c|c|c|c|}
\hline Entry & Deviation from standard conditions & $\begin{array}{c}\text { Conversion } \\
(\%)^{\mathrm{a}}\end{array}$ & $\begin{array}{l}\text { Yield } \\
(\%)^{b}\end{array}$ & er. $^{\mathrm{c}}$ \\
\hline 1 & none & 90 & 88 & $96: 4$ \\
\hline 2 & No $N$-ethylmaleimide & 10 & 0 & - \\
\hline 3 & Norbornadiene instead of $N$-ethylmaleimide & 0 & 0 & - \\
\hline 4 & Acetone instead of $N$-ethylmaleimide & 0 & 0 & - \\
\hline 5 & Ethyl methacrylate instead of $N$-ethylmaleimide & 6 & 0 & - \\
\hline 6 & Diethyl fumarate instead of $N$-ethylmaleimide & 27 & 33 & $96: 4$ \\
\hline 7 & Diethyl maleate instead of $N$-ethylmaleimide & 26 & 32 & $97: 3$ \\
\hline 8 & 0.25 equiv sodium acetate & 22 & 20 & - \\
\hline 9 & 1 equiv sodium acetate & 12 & 8 & - \\
\hline 10 & 0.05 equiv $\mathrm{NaOH}$ instead of sodium acetate & 18 & 0 & - \\
\hline 11 & No sodium acetate & 9 & 0 & - \\
\hline 12 & $\begin{array}{c}5 \mathrm{~mol} \%\left[\mathrm{Ru}(\mathrm{OAc})_{2}(p \text {-cymeme })\right] \text { instead of } \\
\left.\mathrm{RuCl}_{2}(\mathrm{cymene})\right]_{2} \text { and NaOAc }\end{array}$ & 0 & 0 & - \\
\hline 13 & $\begin{array}{l}5 \mathrm{~mol} \%\left[\mathrm{Ru}(\mathrm{OAc})_{2}(p \text {-cymeme })\right] \text { and } 10 \mathrm{~mol} \% \\
\left.\left.\text { TBA-Cl instead of } \mathrm{RuCl}_{2} \text { (cymene }\right)\right]_{2} \text { and } \mathrm{NaOAc}\end{array}$ & 52 & 52 & $96: 4$ \\
\hline 14 & $\begin{array}{c}(R) \text {-binap (5 mol\%) instead of rac-binap; no } \\
\left.\left.\mathrm{RuCl}_{2} \text { (cymene) }\right]_{2} \text { and }(S, S) \text {-me-bipam in step } 2\right)\end{array}$ & 86 & 0 & - \\
\hline 15 & $\begin{array}{c}5.5 \text { mol\% }(S, S) \text {-me-bipam in step 1) instead of } \\
\text { rac-binap and NaOAc; benzyl alcohol instead of } \\
\text { hexan-1-ol; 4-methoxyphenylboronic acid } \\
\text { instead of phenylboronic acid }\end{array}$ & 0 & 0 & - \\
\hline 16 & $\mathrm{PPh}_{3}(10 \mathrm{~mol} \%)$ instead of $\mathrm{rac}$-binap & 36 & 30 & $58: 42$ \\
\hline 17 & dppf (5 mol\%) instead of rac- binap & 31 & 20 & $94: 6$ \\
\hline 18 & xantphos $(5 \mathrm{~mol} \%)$ instead of $\mathrm{rac}$-binap & 5 & 5 & - \\
\hline 19 & dpephos (5 mol\%) nstead of $r a c$-binap & 46 & 39 & $96: 4$ \\
\hline 20 & $(R)$-binap $(5 \mathrm{~mol} \%)$ instead of $\mathrm{rac}$-binap & 87 & 91 & $96: 4$ \\
\hline 21 & $(S)$-binap $(5 \mathrm{~mol} \%)$ instead of $r a c$-binap & 89 & 90 & $96: 4$ \\
\hline 22 & 1,4-dioxane instead of toluene in step 1) & 27 & 0 & - \\
\hline 23 & THF instead of toluene in step 1) & 73 & 0 & - \\
\hline 24 & Hexane instead of toluene in step 1) & 28 & 0 & - \\
\hline 25 & DCM instead of toluene in step 1) & 80 & 58 & $95: 5$ \\
\hline 26 & $\begin{array}{c}\left.1.25 \mathrm{~mol} \% \mathrm{RuCl}_{2} \text { (cymene) }\right]_{2}, 2.75 \mathrm{~mol} \%(S, S) \text { - } \\
\text { me-bipam in step 2) }\end{array}$ & 88 & 77 & $96: 4$ \\
\hline 27 & $\begin{array}{c}\left.0.5 \mathrm{~mol}^{2} \mathrm{RuCl}_{2} \text { (cymene) }\right]_{2}, 1.1 \mathrm{~mol} \%(S, S) \text {-me- } \\
\text { bipam in step 2) }\end{array}$ & 89 & 68 & $96: 4$ \\
\hline 28 & $\begin{array}{l}\left.1.25 \mathrm{~mol}_{\%} \mathrm{RuCl}_{2}(\mathrm{cymene})\right]_{2}, 2.5 \mathrm{~mol} \% \mathrm{rac}- \\
\text { binap, } 2.5 \mathrm{~mol} \% \mathrm{NaOAc} \text { in step } 1)\end{array}$ & 54 & 50 & $96: 4$ \\
\hline
\end{tabular}




\begin{tabular}{|c|c|c|c|c|}
\hline 29 & $\begin{array}{c}\left.1.25 \mathrm{~mol}^{2} \mathrm{RuCl}_{2} \text { (cymene) }\right]_{2}, 2.5 \mathrm{~mol} \% \mathrm{rac} \text { - } \\
\text { binap, } 2.5 \mathrm{~mol} \% \mathrm{NaOAc} \text { in step } 1) \\
\left.1.25 \mathrm{~mol}^{2} \mathrm{RuCl}_{2} \text { (cymene) }\right]_{2}, 2.75 \mathrm{~mol} \%(S, S)- \\
\text { me-bipam in step } 2)\end{array}$ & 45 & 43 & $96: 4$ \\
\hline 30 & $\begin{array}{c}\left.0.5 \mathrm{~mol} \% \mathrm{RuCl}_{2} \text { (cymene) }\right]_{2}, 1 \text { mol\% rac-binap, } \\
1 \mathrm{~mol} \% \mathrm{NaOAc} \text { in step } 1 \text { ) }\end{array}$ & 12 & 13 & - \\
\hline 31 & $\begin{array}{c}\left.0.5 \mathrm{~mol} \% \mathrm{RuCl}_{2} \text { (cymene) }\right]_{2}, 1 \mathrm{~mol} \% \text { rac-binap, } \\
\left.1 \mathrm{~mol}^{2} \mathrm{NaOAc} \text { in step } 1\right) \text {, } \\
\left.0.5 \mathrm{~mol} \% \mathrm{RuCl}_{2} \text { (cymene) }\right]_{2}, 1.1 \mathrm{~mol} \%(S, S) \text {-me- } \\
\text { bipam in step } 2 \text { ) }\end{array}$ & 12 & 11 & - \\
\hline
\end{tabular}

${ }^{a}$ Conversions determined by GC-FID analysis with dodecane as an internal standard. GC conversions were corrected for response factors for all compounds (See section V. Calibration Curves for GC Analysis). The estimated error of $\pm 2 \%$ of the value. ${ }^{b}$ Yields determined by ${ }^{1} \mathrm{H}$ NMR analysis with 1,3,5-trimethoxybenzene as an internal standard. The estimated error of \pm $5 \%$ of the value. ${ }^{c}$ Enantiomeric ratios determined by SFC analysis on a chiral stationary phase.<smiles>c1ccc(-c2cccc3cccc(-c4c5cccc-5ccc5ccccc45)c23)cc1</smiles>

rac-binap<smiles>CC1(C)c2ccccc2Oc2c(P)cccc21</smiles><smiles>Pc1ccccc1Oc1ccccc1P</smiles>

xantphos<smiles>CNP(NC)Oc1c(COCc2cc3ccccc3c(-c3c(OP(NC)Oc4ccc5ccccc5c4)ccc4ccccc34)c2-c2ccccc2)ccc2ccccc12</smiles>

$(S, S)$-me-bipam b)

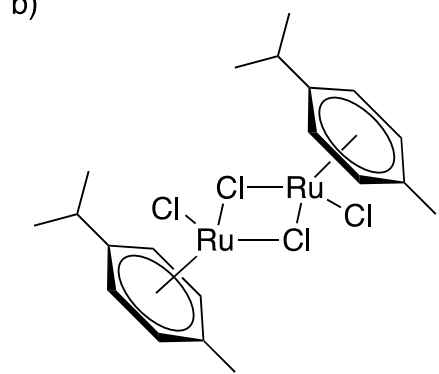

$\left[\mathrm{RuCl}_{2}(p-\text { cymene })\right]_{2}$

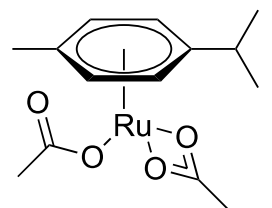

[Ru(OAc) $\left.)_{2}(p-c y m e n e)\right]$

c)<smiles>C1=CC2C=CC1C2</smiles><smiles>C=C(C)C(=O)OCC</smiles>

Norbornadiene Ethyl Methacrylate $\mathrm{N}$-Ethylmaleimide<smiles>CCOC(=O)/C=C\C(=O)OCC</smiles>

Diethyl Maleate<smiles>CCOC(=O)/C=C/C(=O)OCC</smiles>

Diethyl Fumarate

Figure S1. a) Structures of evaluated ligands. b) Structure of used metal sources. c) Structures of evaluated sacrificial hydrogen acceptors. 


\section{Unsuccessful examples}<smiles>CCC/C=C/CO</smiles><smiles>C#CCCCCCO</smiles><smiles>CCC/C=C\CCO</smiles><smiles>CNCCCO</smiles><smiles>Cn1ccc2cc(BO)ccc21</smiles><smiles>CC(C)(C)OCc1ccc(NC(=O)OCc2ccccc2)cc1</smiles><smiles>[18OH]c1cccs1</smiles><smiles>COc1ccc(SC)cc1</smiles><smiles>[18O]c1ccco1</smiles>

Figure S2. Examples of starting materials of the unsuccessful reactions. 


\section{Calibration Curves for GC Analysis}
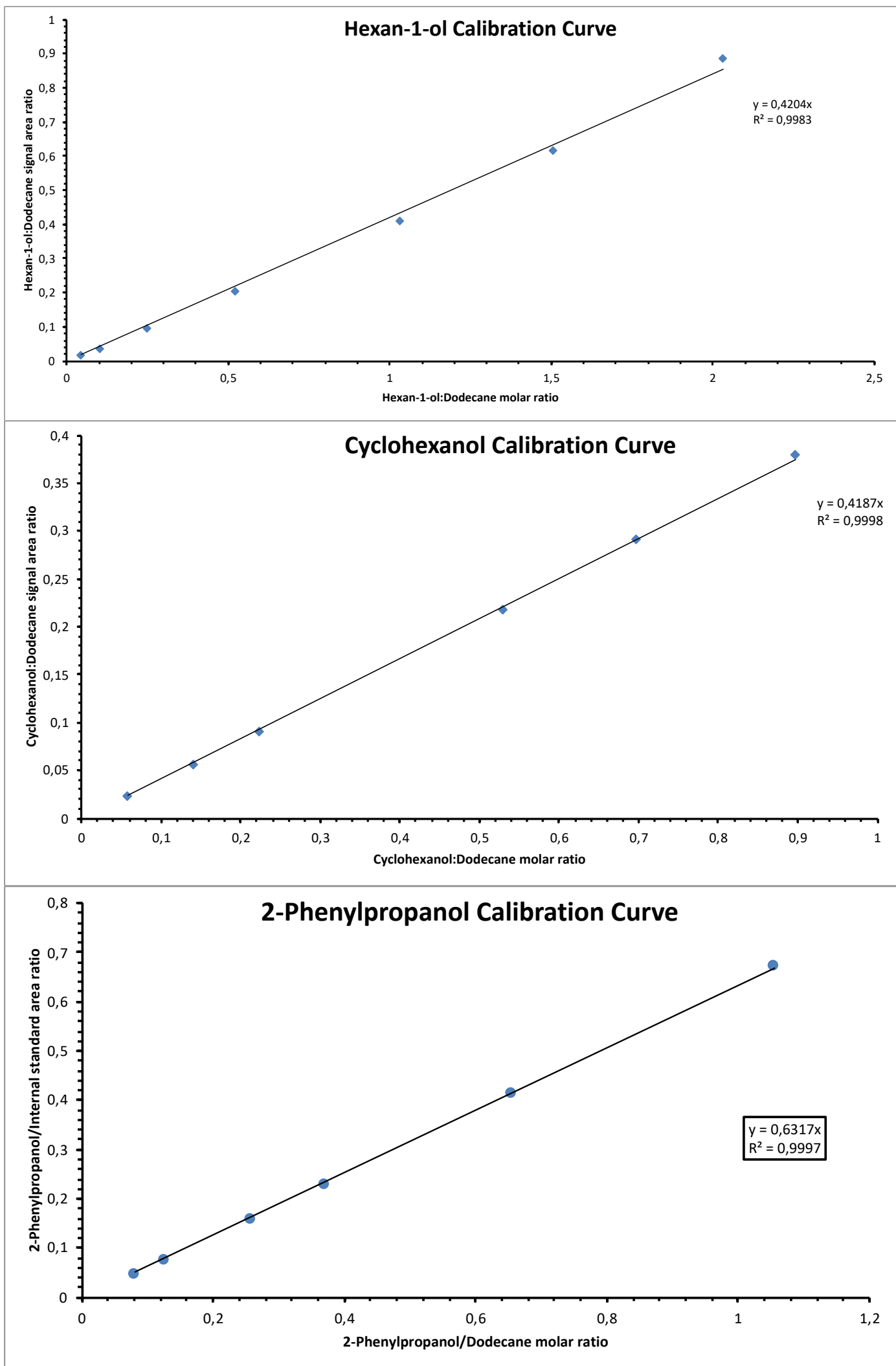

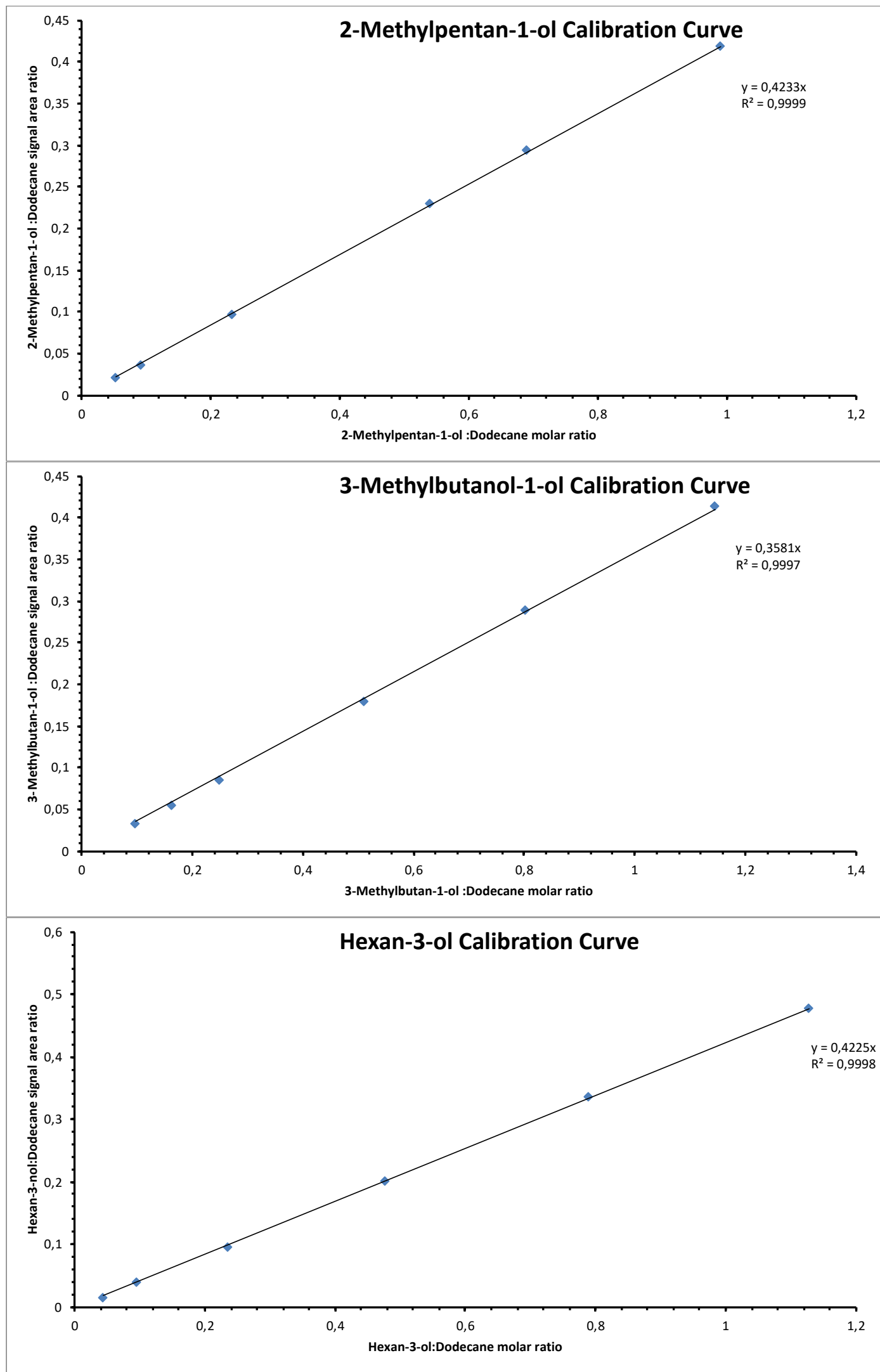


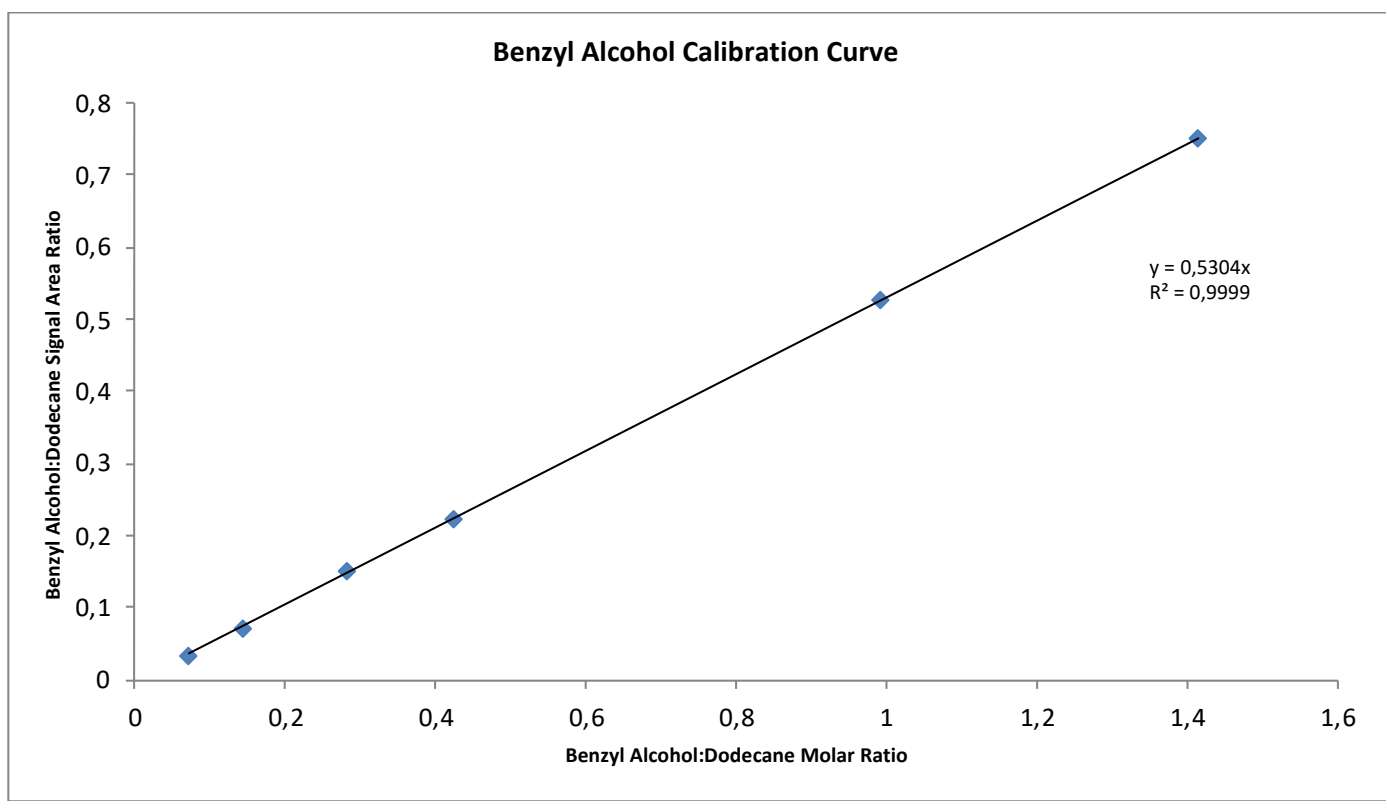




\section{NMR Spectra}

\section{IV.A NMR Spectra of the Prepared Substrates}

ڤ్<smiles>CC(C)OC(=O)CCCCCO</smiles>

$1 \mathbf{k}$

${ }^{1} \mathrm{H}$ NMR

$(400 \mathrm{MHz}, \mathrm{CDCl} 3)$

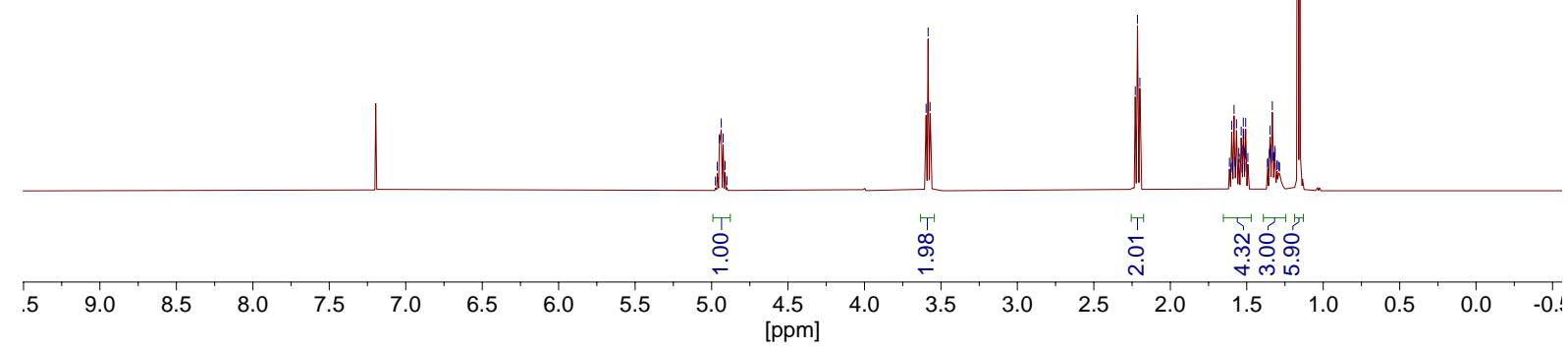

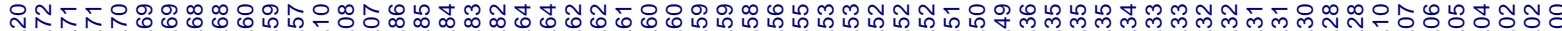

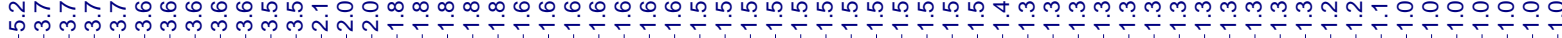

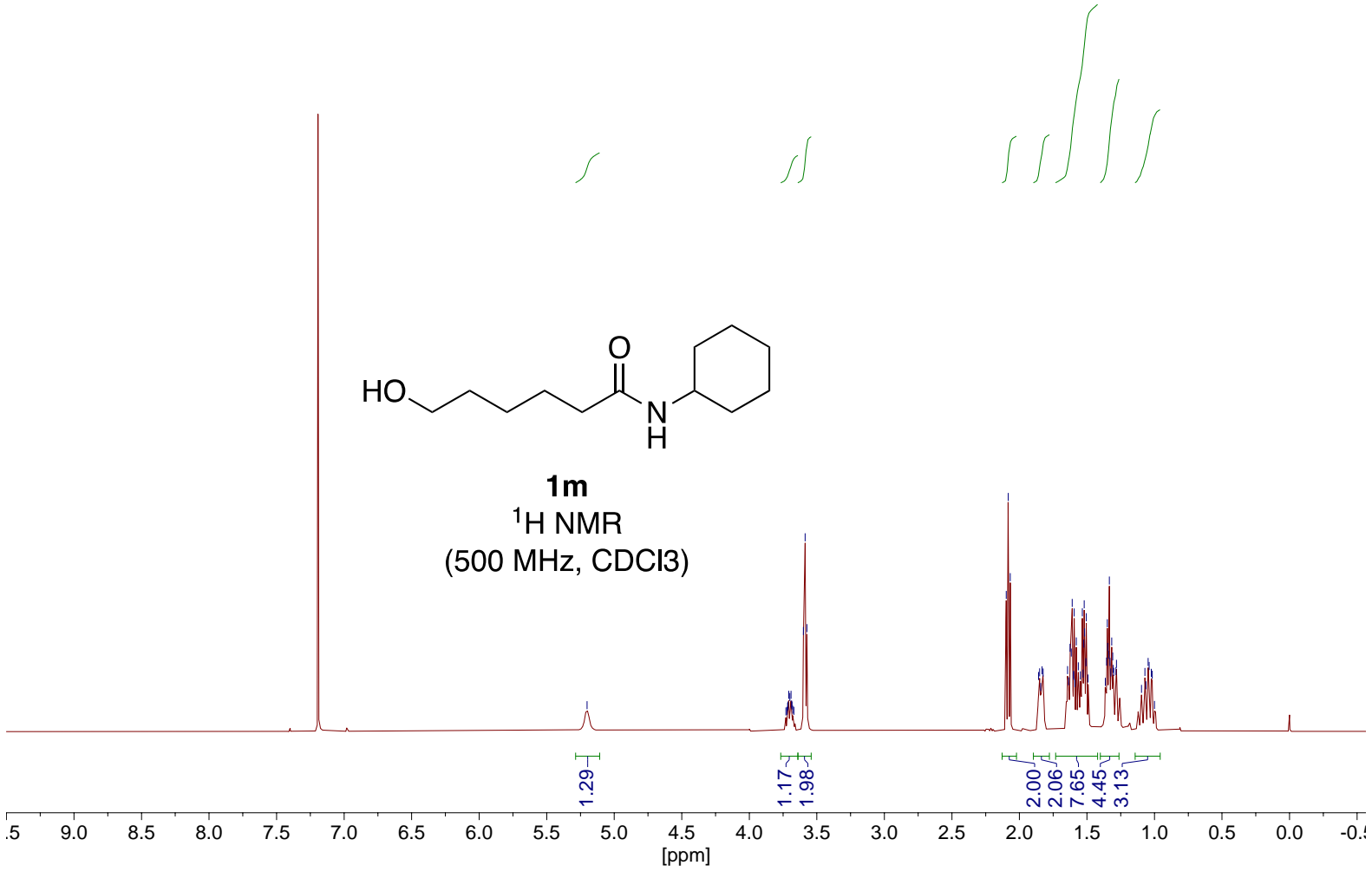




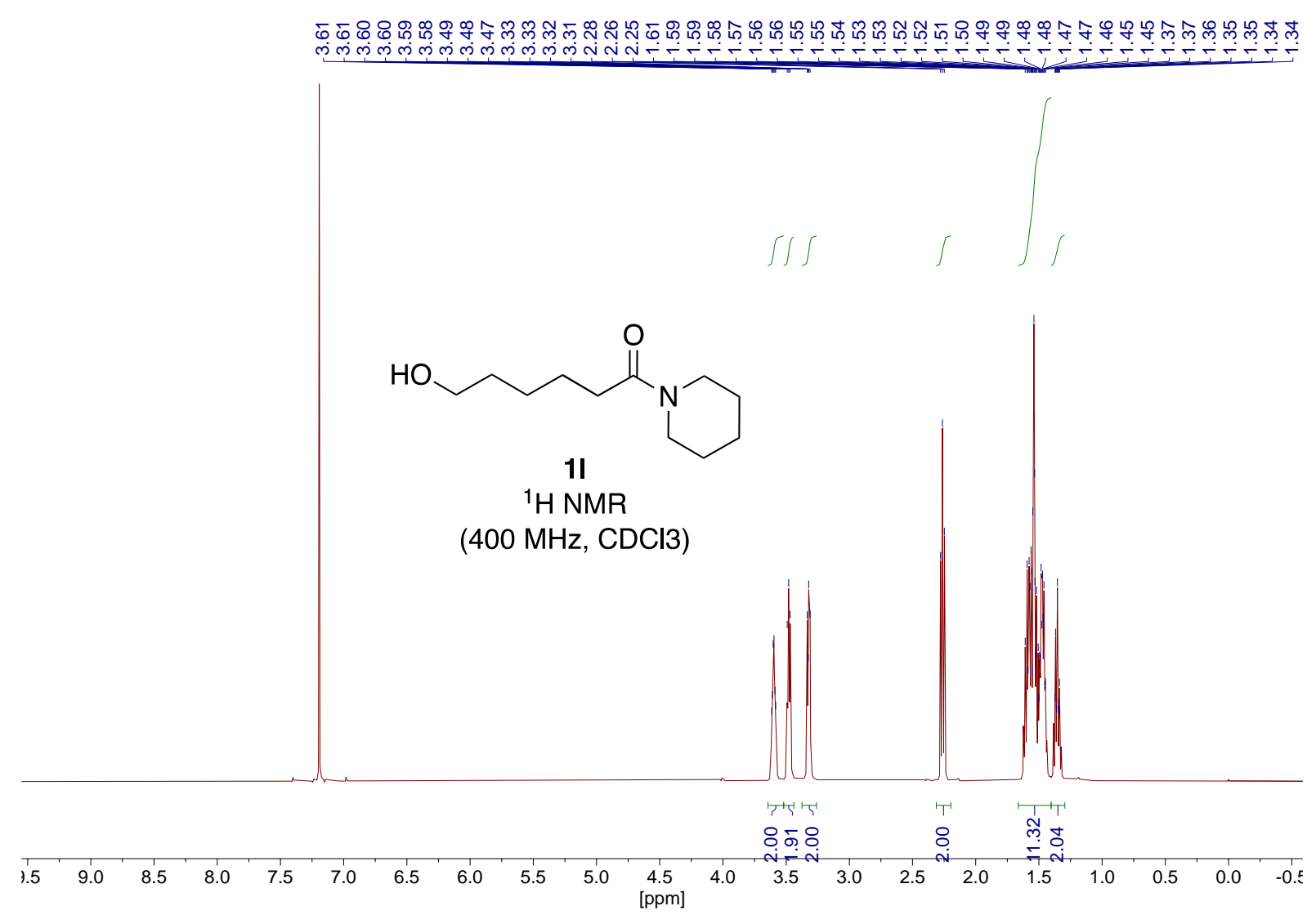

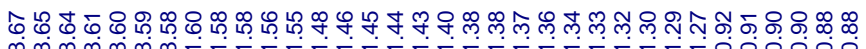

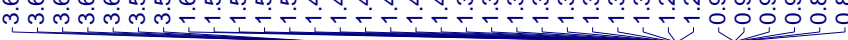<smiles>CCCCCCC(O)CCCCCCCCCCCO</smiles>

$1 p$

${ }^{1} \mathrm{H}$ NMR

(400 MHz, CDCl3)

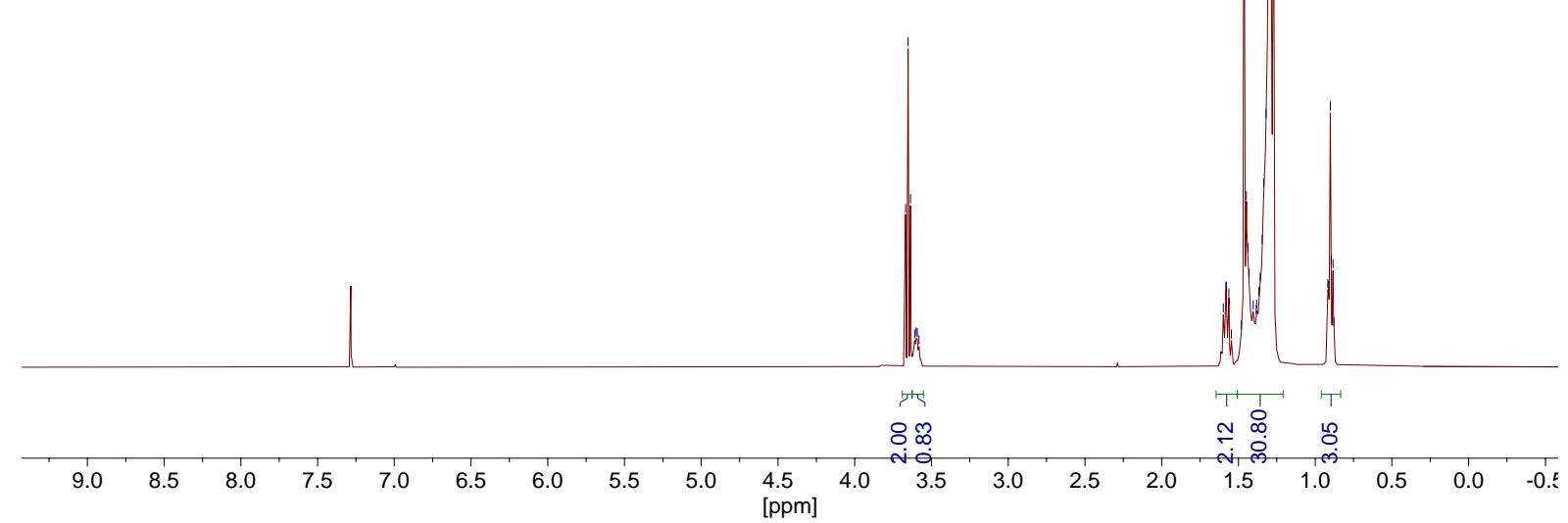




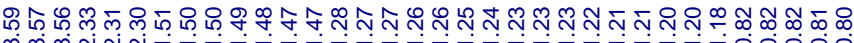
- 0000
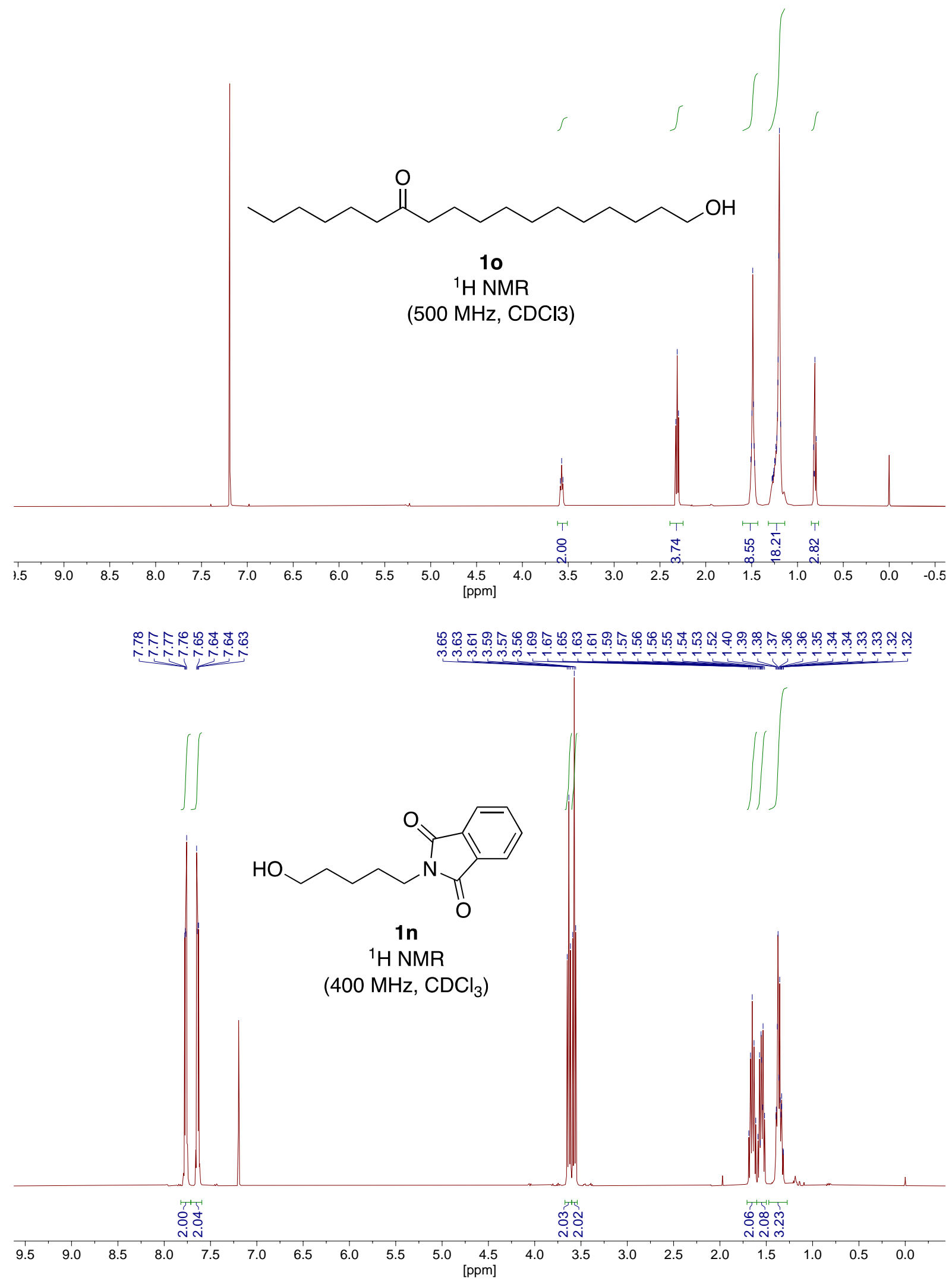


\section{IV.B NMR Spectra of the Products}
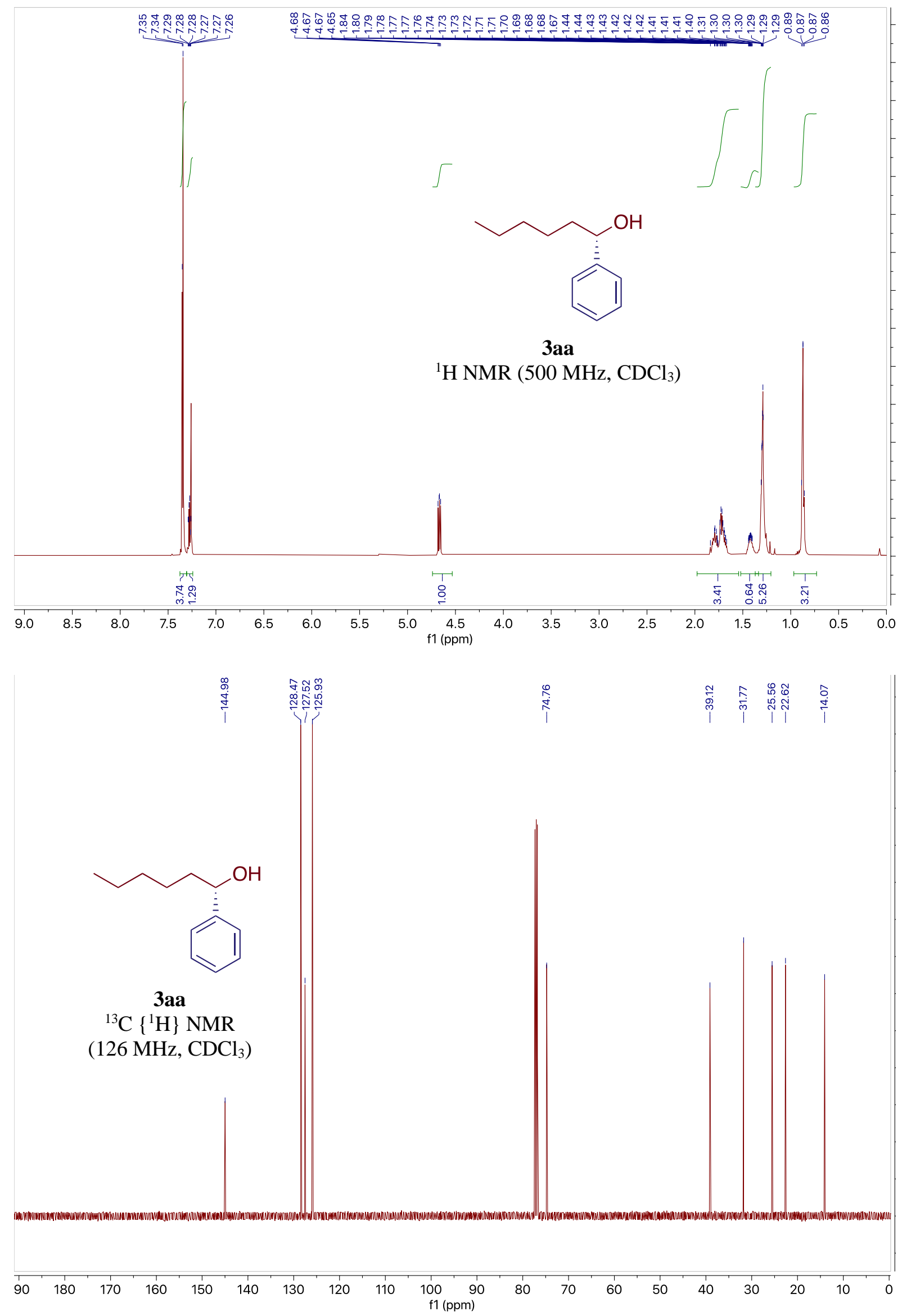


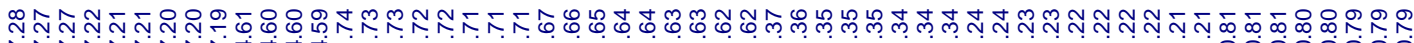

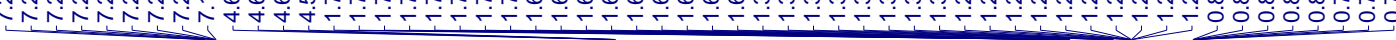

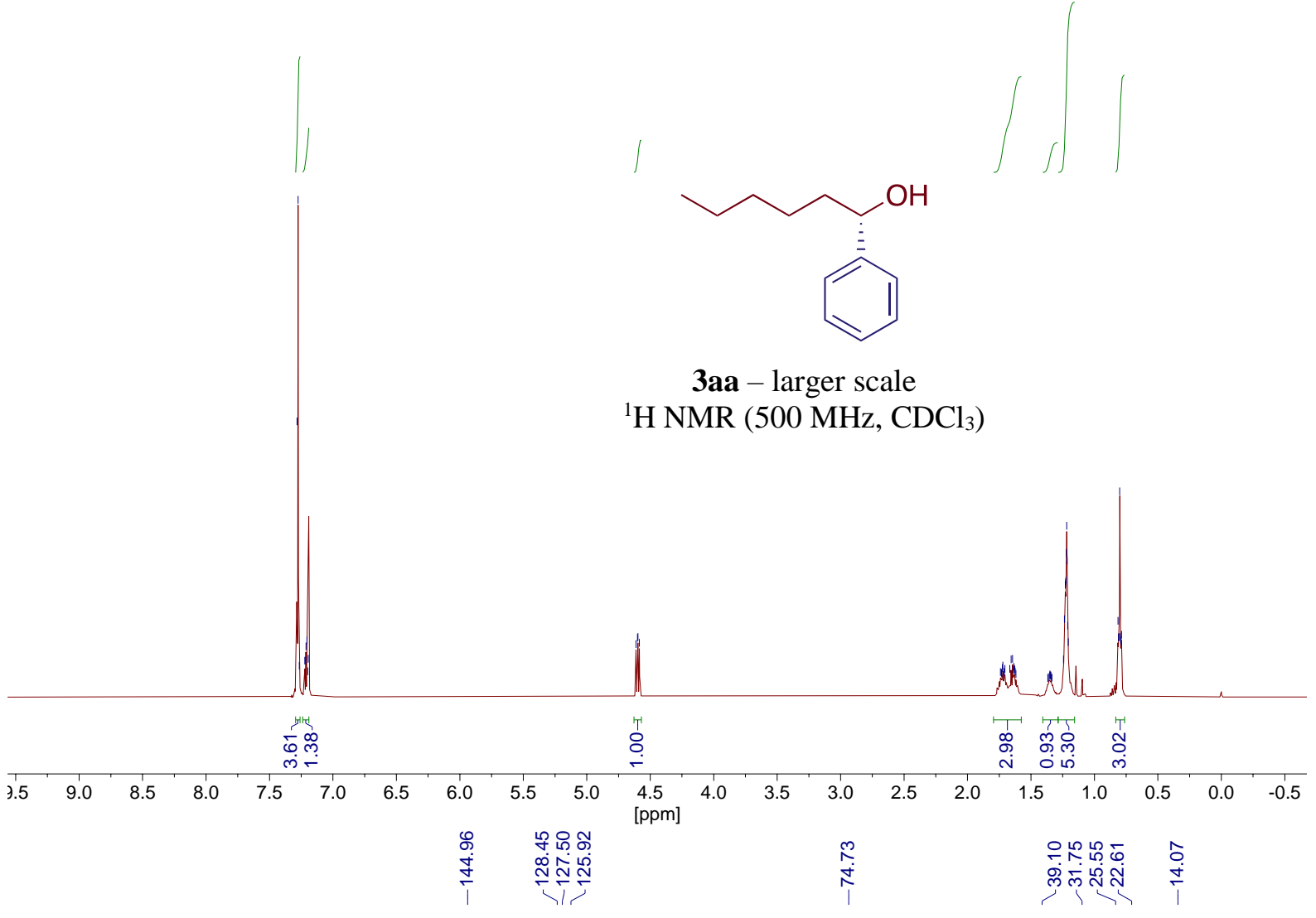<smiles>CCCCCC(O)c1ccccc1</smiles>

3aa- larger scale

${ }^{13} \mathrm{C}\left\{{ }^{1} \mathrm{H}\right\}$ NMR

$\left(126 \mathrm{MHz}, \mathrm{CDCl}_{3}\right.$ )

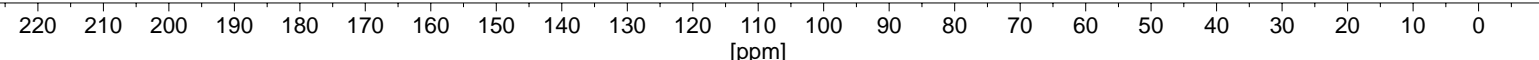



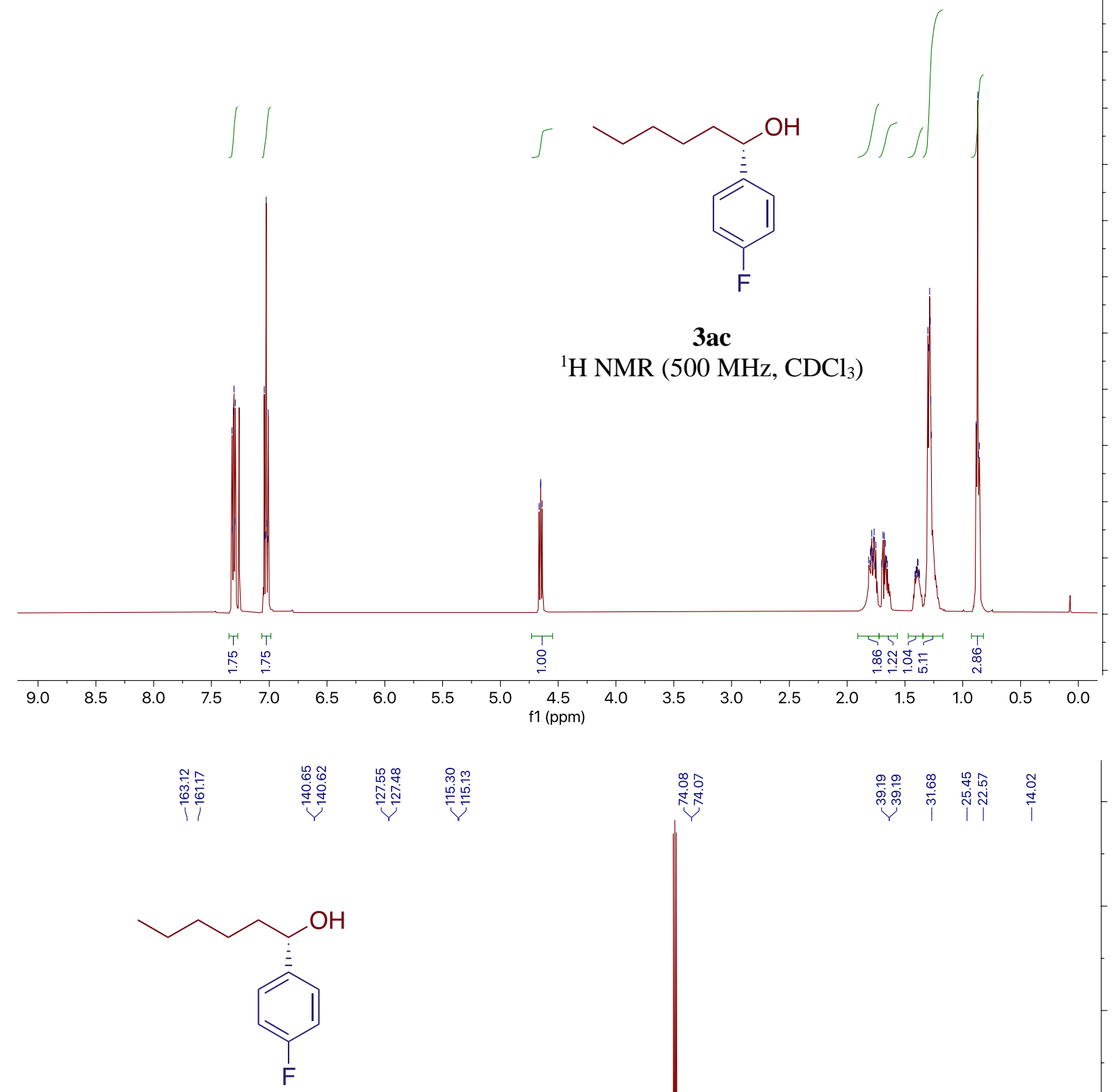

3ac

${ }^{13} \mathrm{C}\left\{{ }^{1} \mathrm{H}\right\} \mathrm{NMR}$

$\left(126 \mathrm{MHz}, \mathrm{CDCl}_{3}\right)$

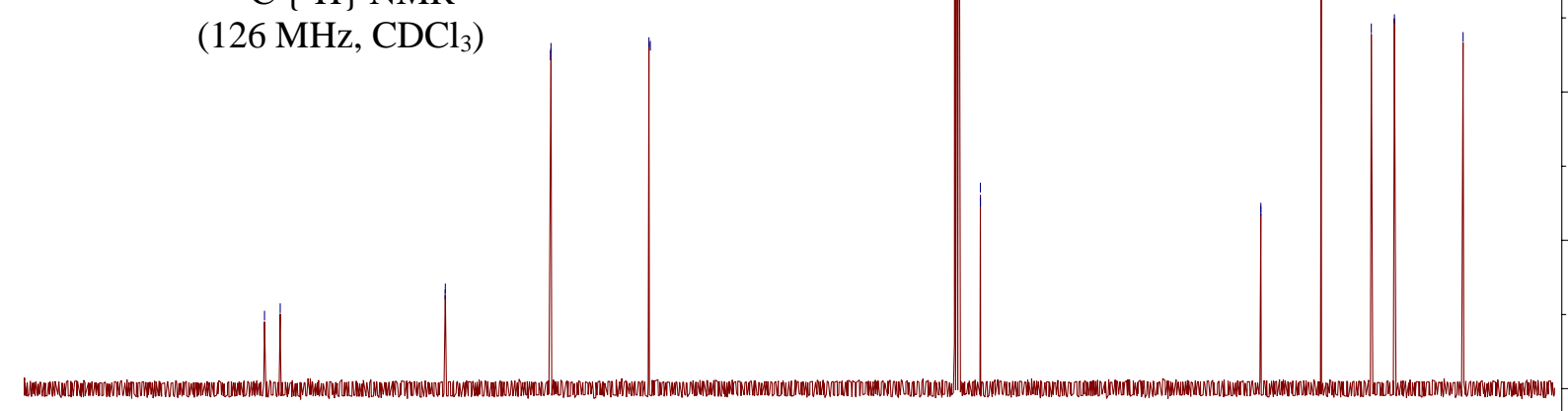

Lwer. 


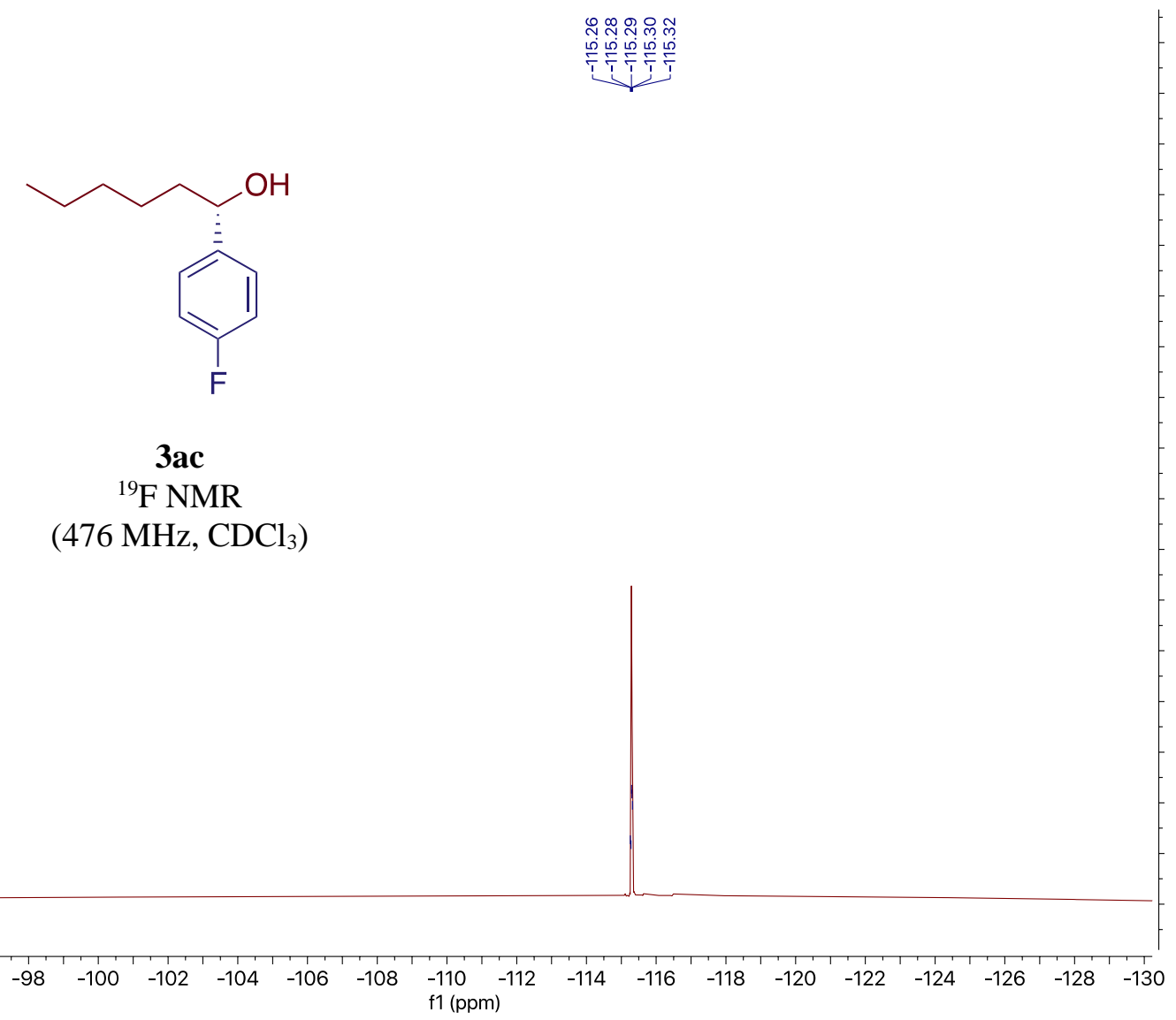



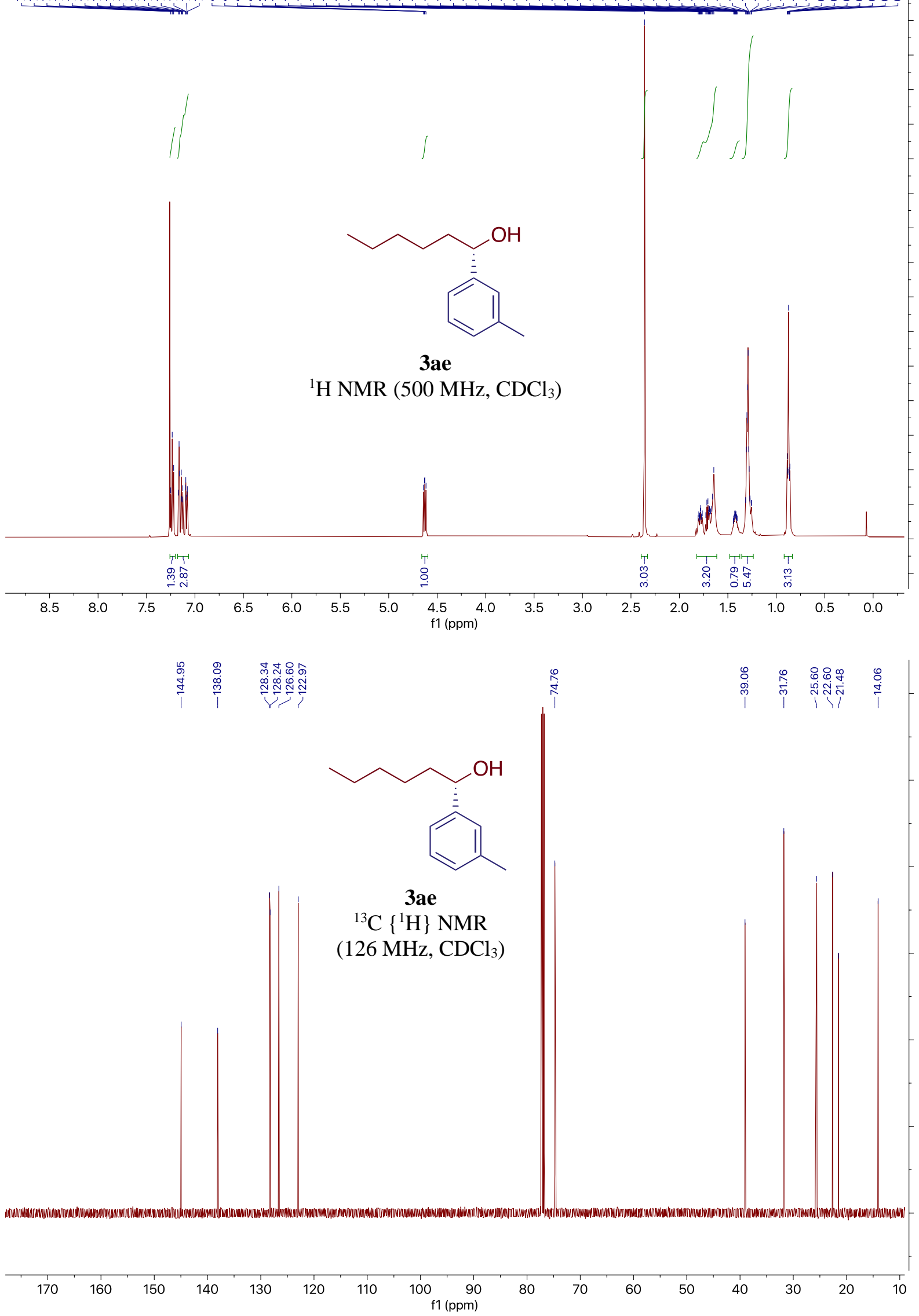


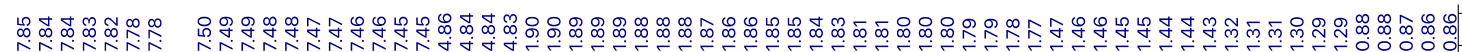

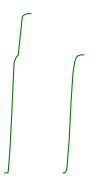<smiles>CCCCCC(O)c1ccc2ccccc2c1</smiles>
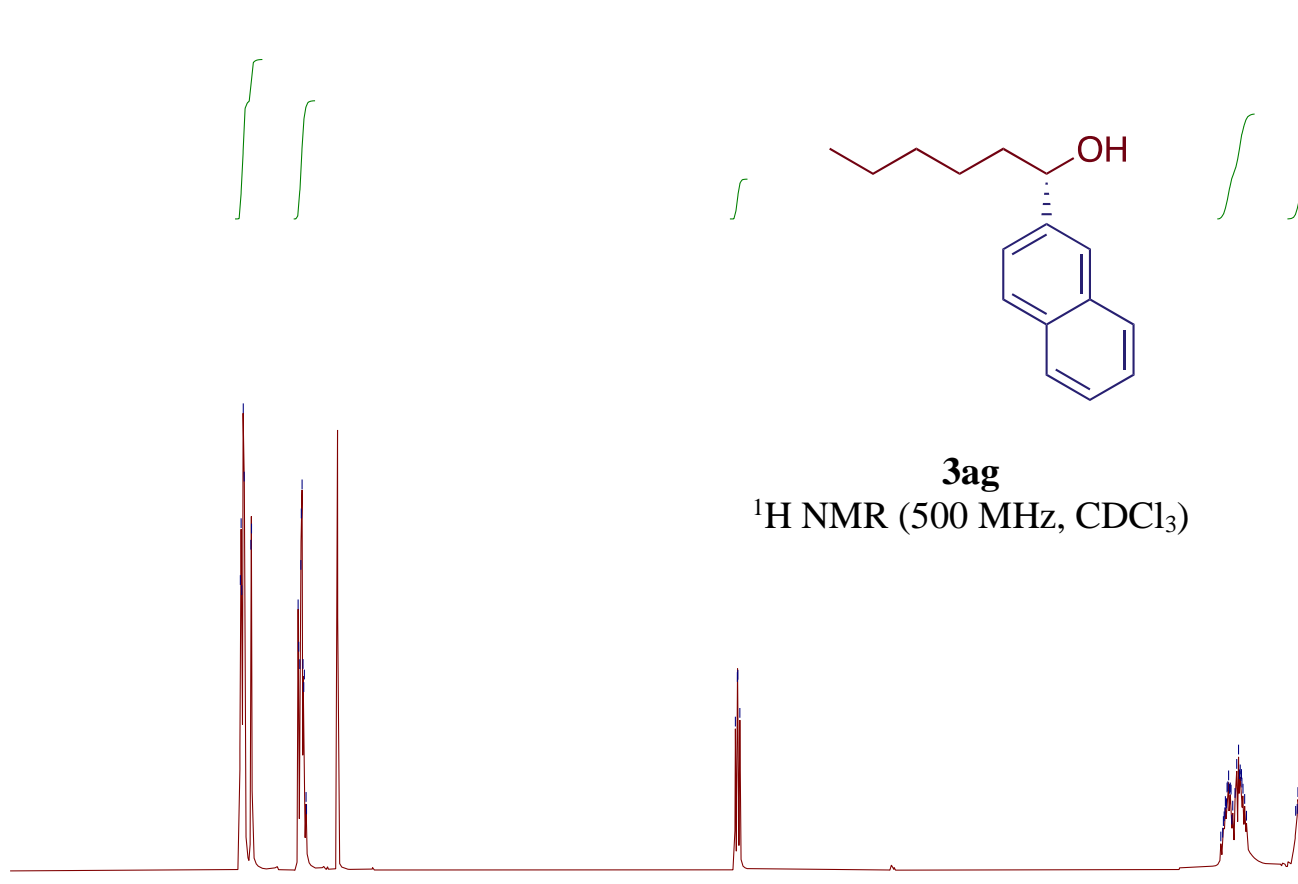

$3 a g$

${ }^{1} \mathrm{H}$ NMR (500 MHz, $\mathrm{CDCl}_{3}$ )

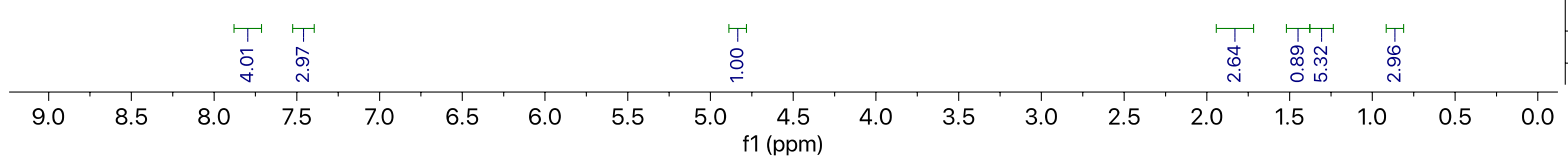

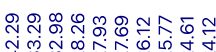

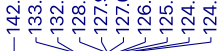

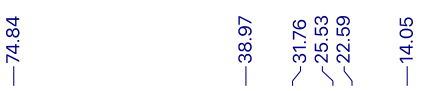<smiles>CCCCCC(O)c1ccc2ccccc2c1</smiles>

3ag

${ }^{13} \mathrm{C}\left\{{ }^{1} \mathrm{H}\right\}$ NMR $\left(126 \mathrm{MHz}, \mathrm{CDCl}_{3}\right)$

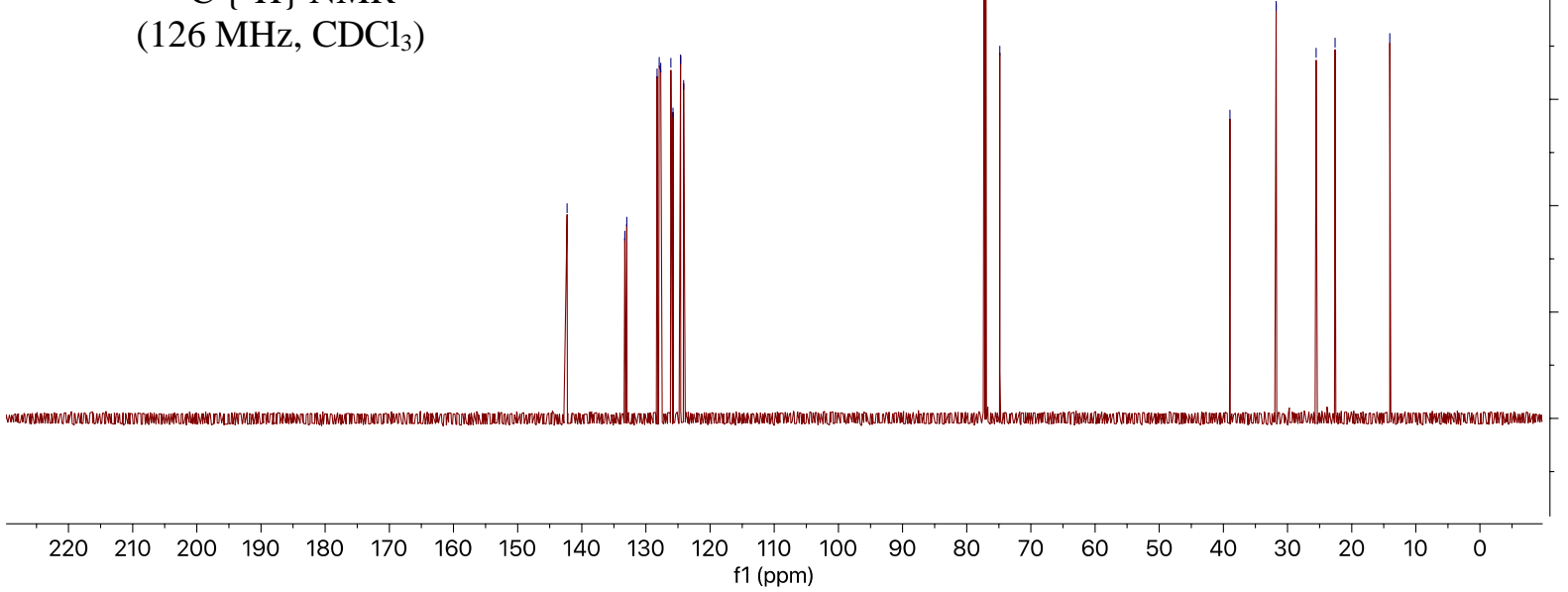



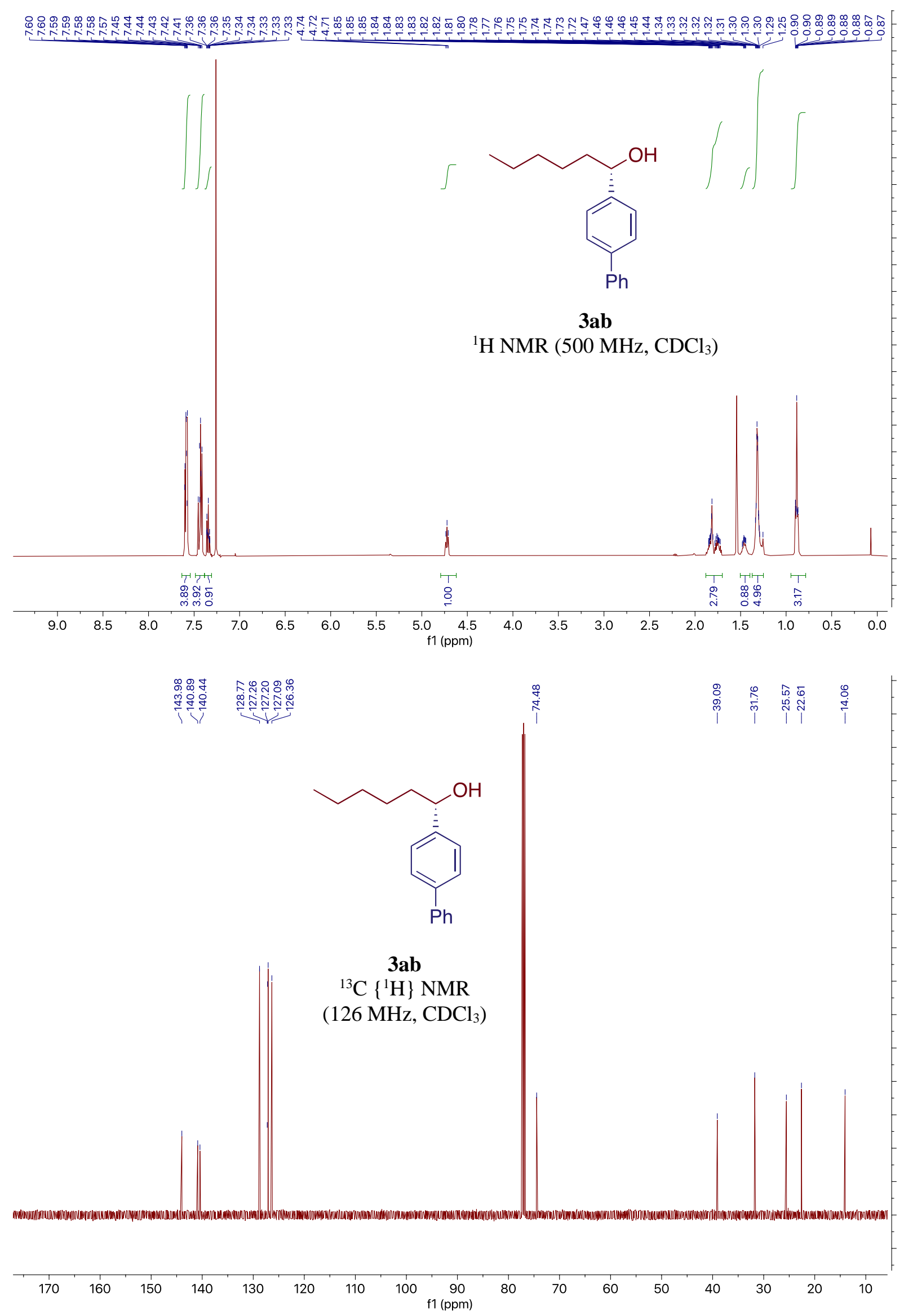


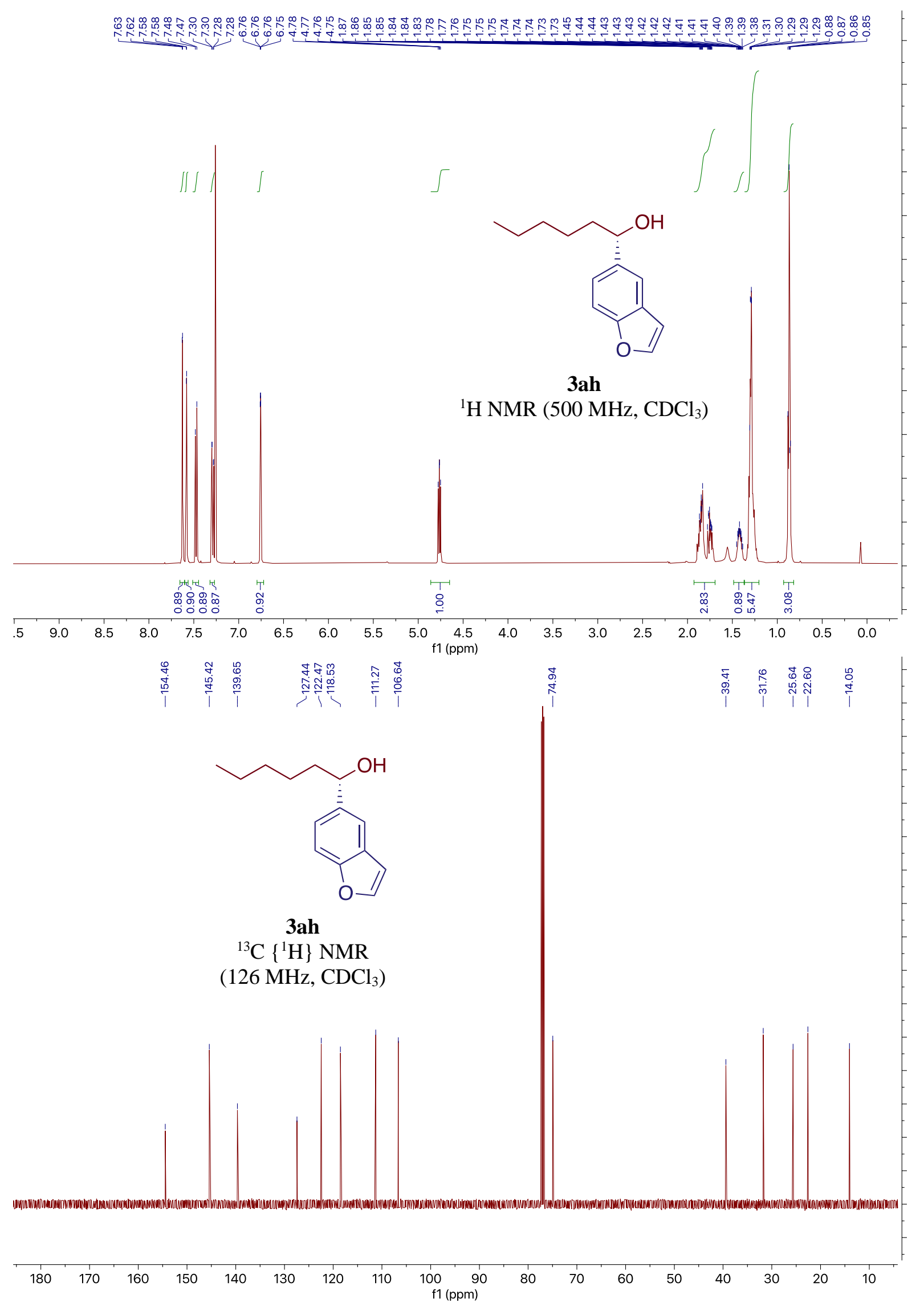




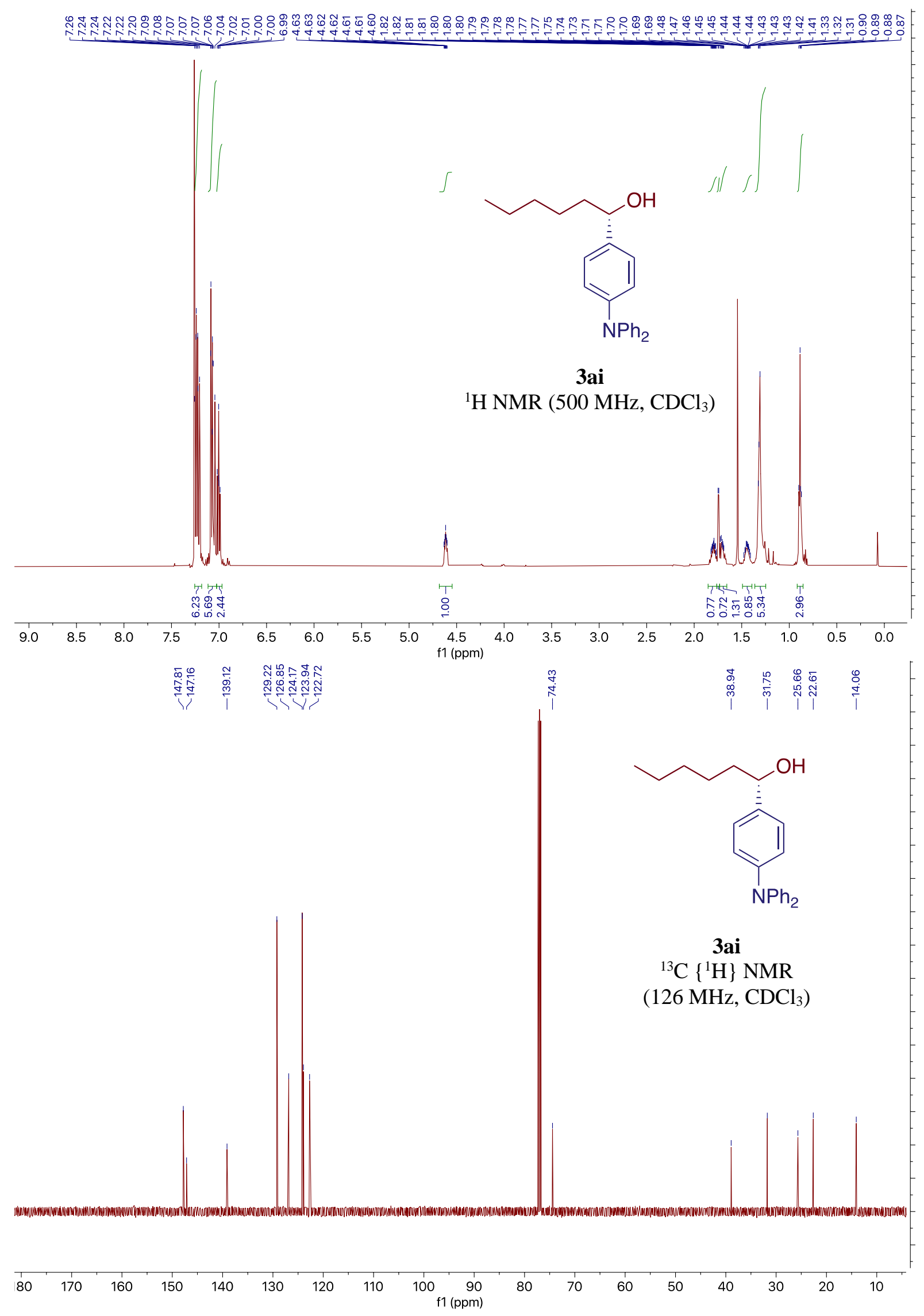




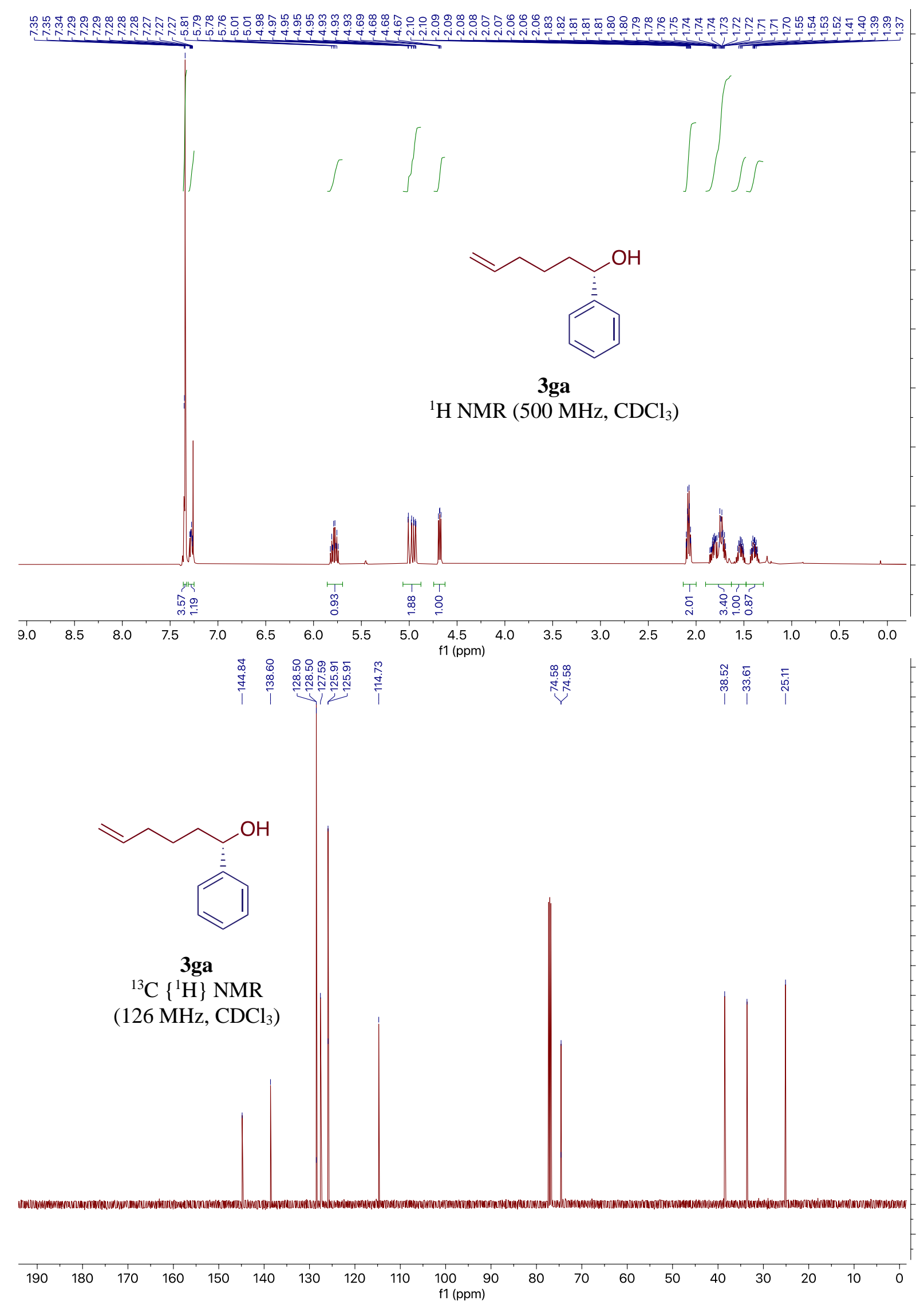




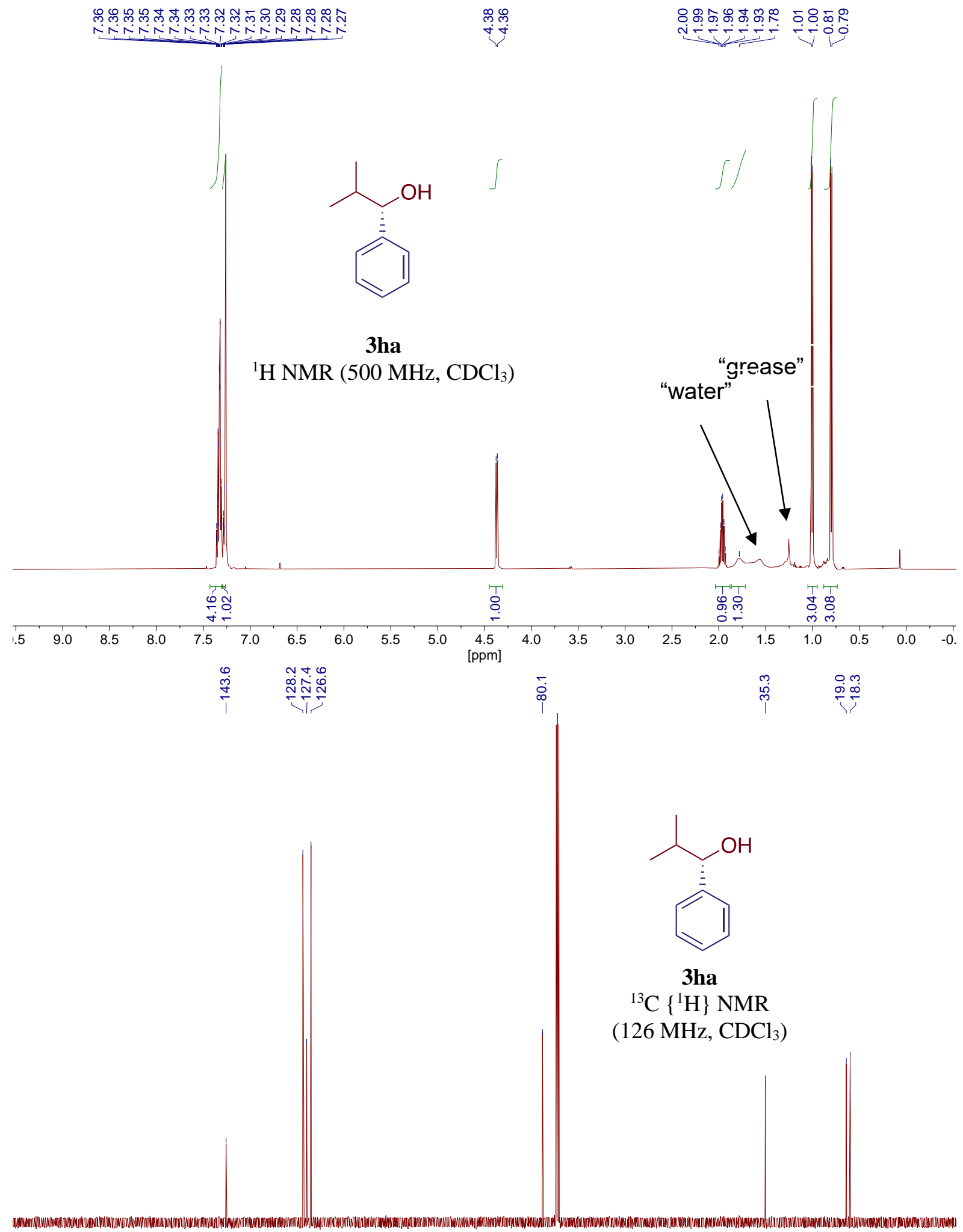

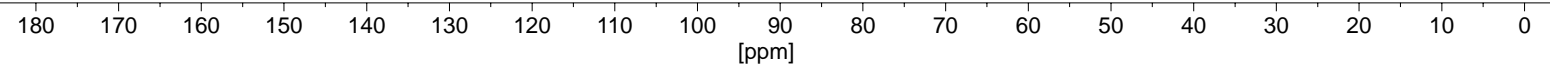



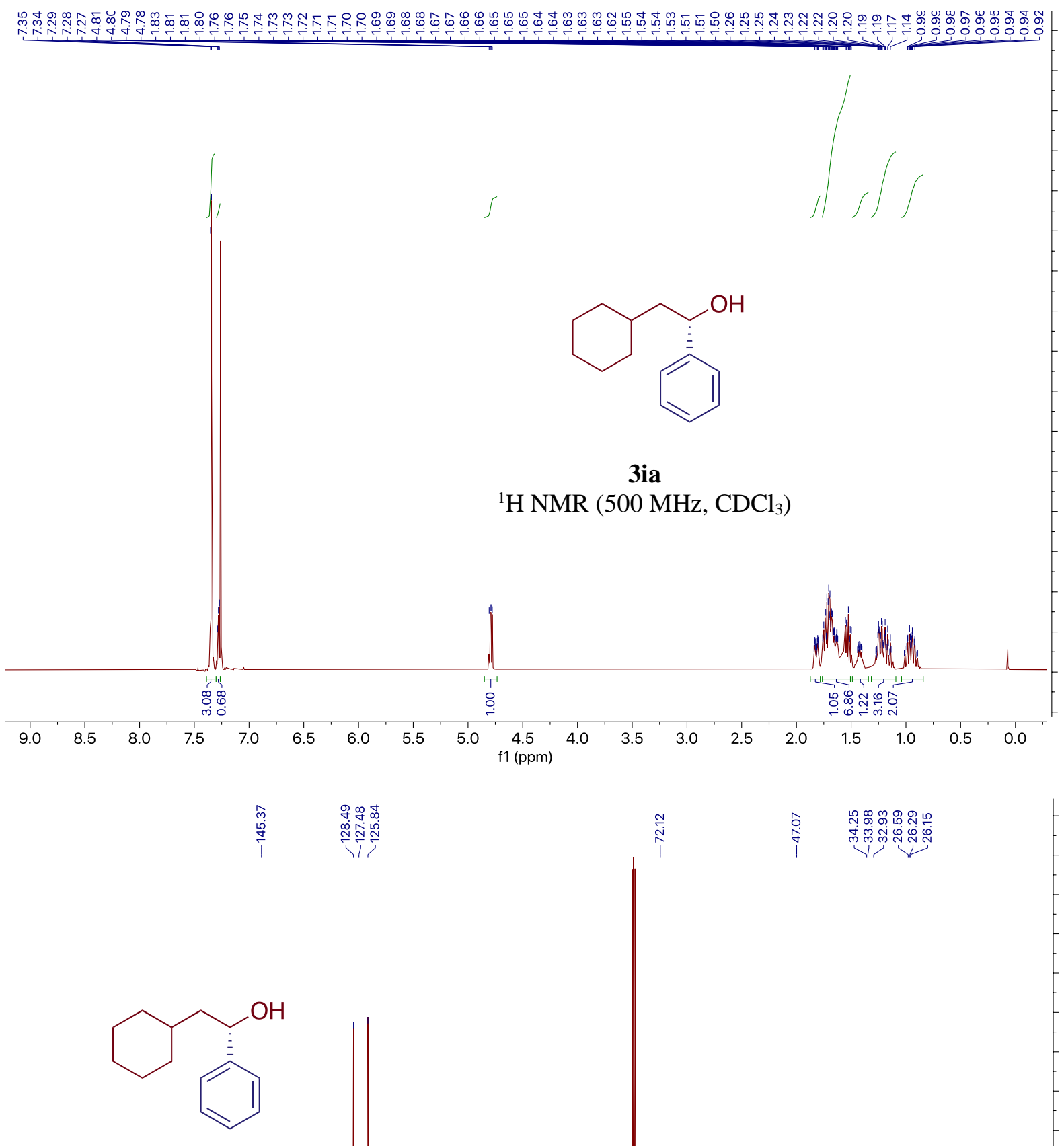

3ia

${ }^{13} \mathrm{C}\left\{{ }^{1} \mathrm{H}\right\} \mathrm{NMR}$ $\left(126 \mathrm{MHz}, \mathrm{CDCl}_{3}\right)$

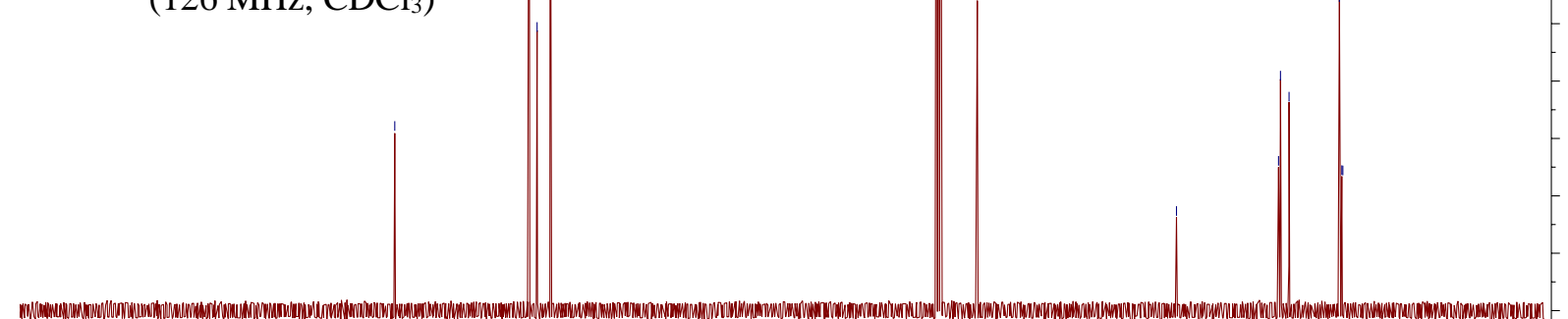

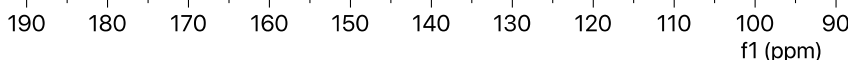




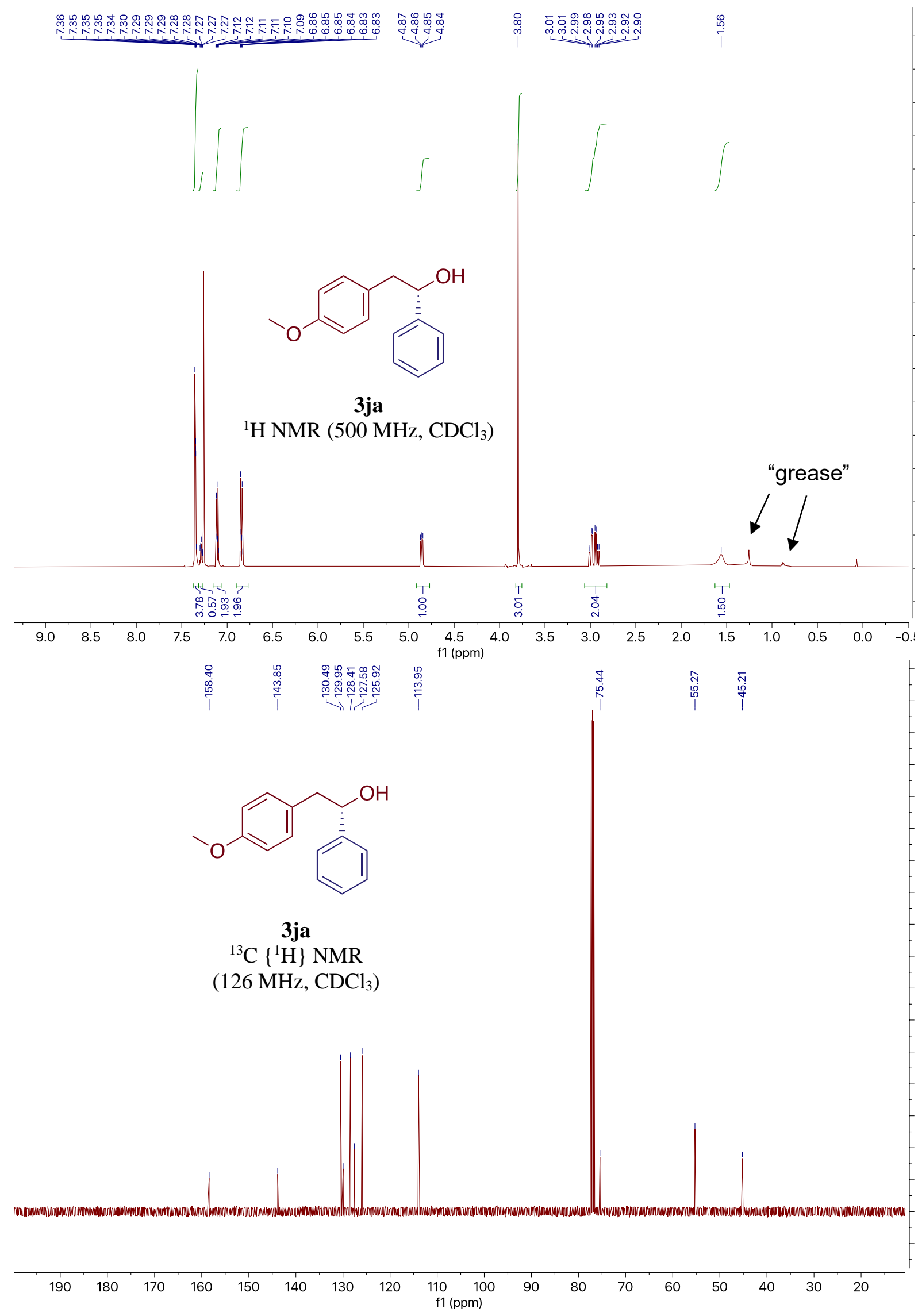




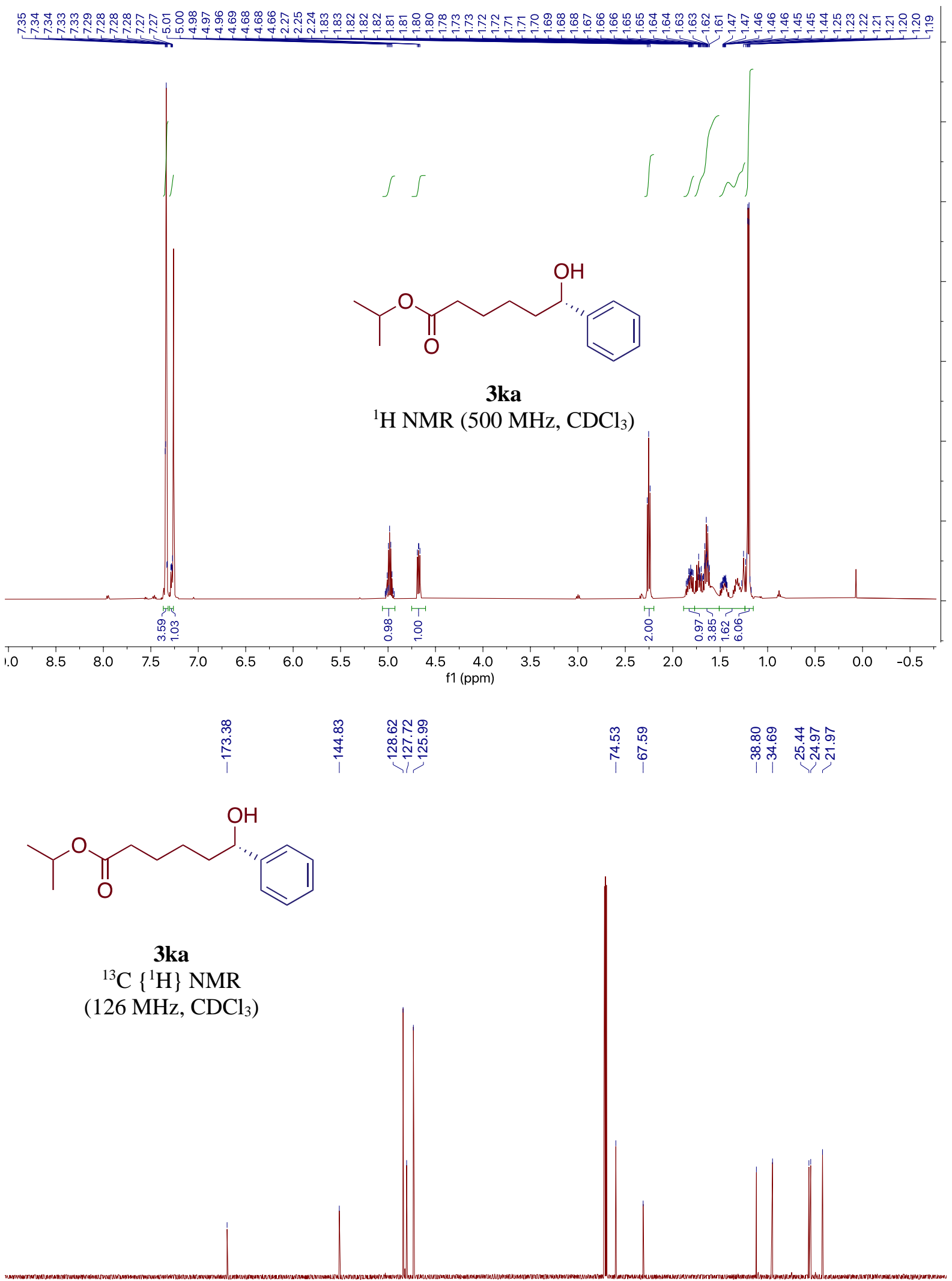

$\begin{array}{lllllllllllllllllllllllll}220 & 210 & 200 & 190 & 180 & 170 & 160 & 150 & 140 & 130 & 120 & \begin{array}{c}110 \\ {[\mathrm{ppm}]}\end{array} & 100 & 90 & 80 & 70 & 60 & 50 & 40 & 30 & 20 & 10 & 0 & \end{array}$ 

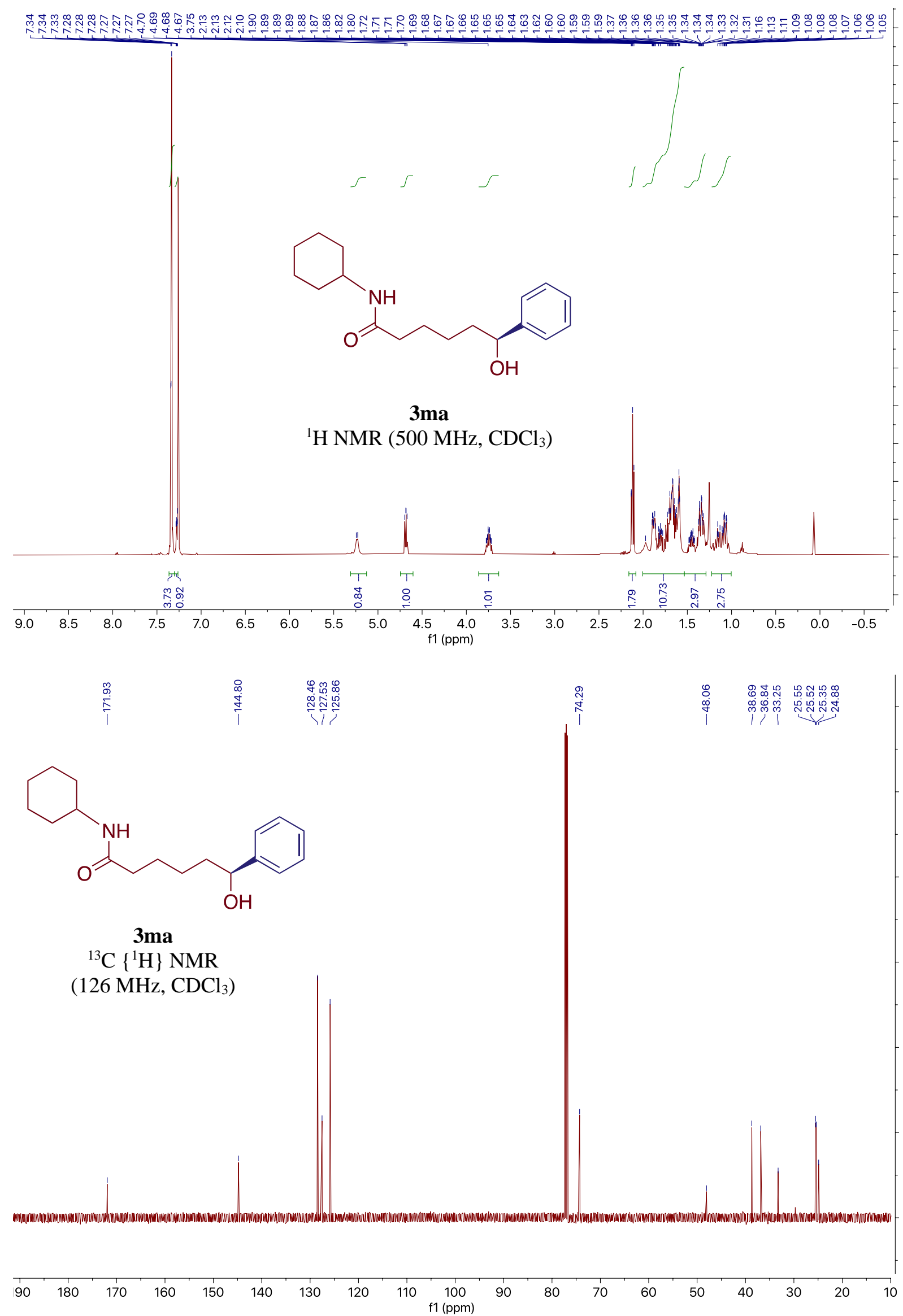


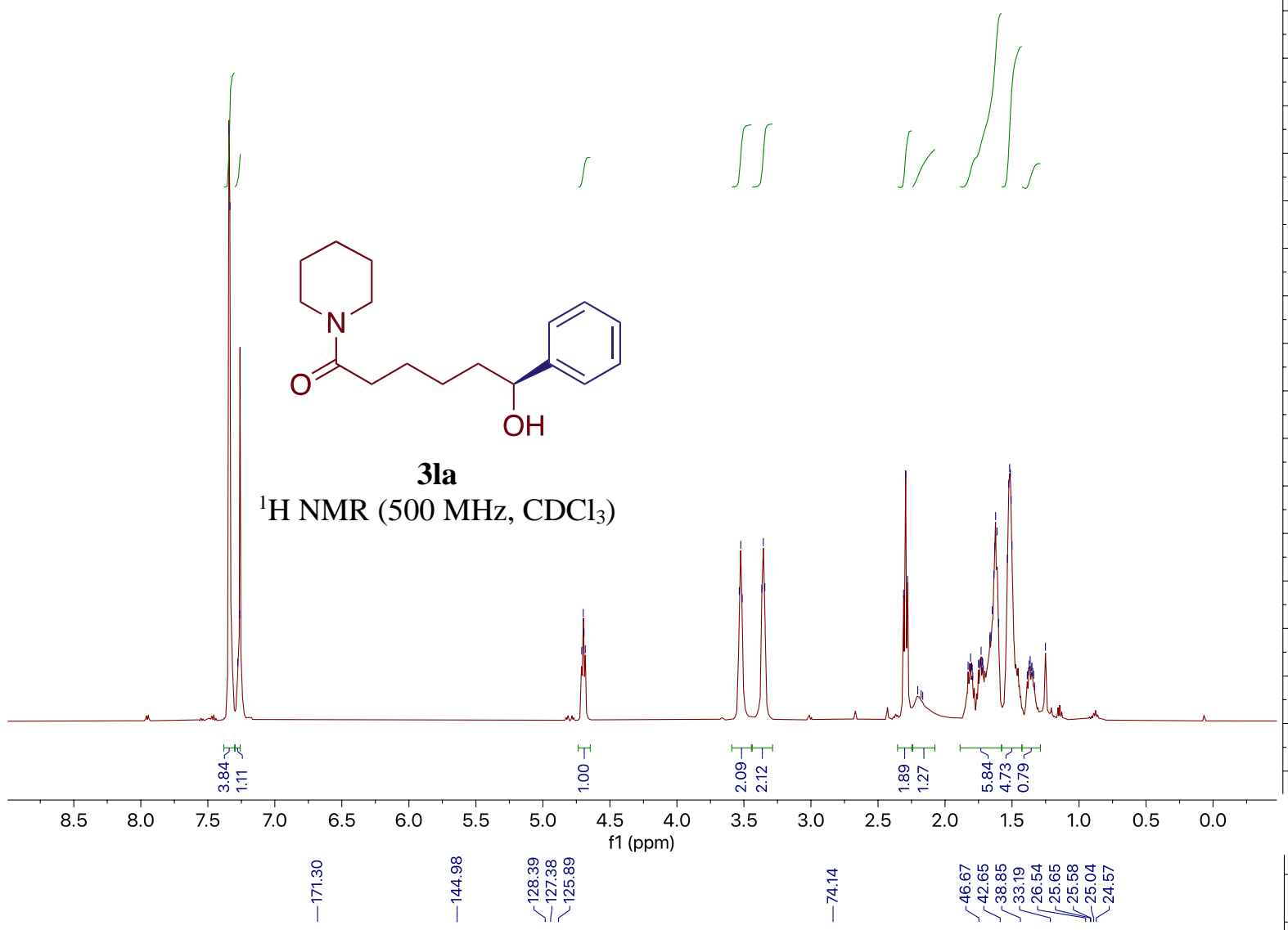<smiles>O=C(CCCCC(O)c1ccccc1)N1CCCCC1</smiles>

3la

${ }^{13} \mathrm{C}\left\{{ }^{1} \mathrm{H}\right\}$ NMR $\left(126 \mathrm{MHz}, \mathrm{CDCl}_{3}\right)$

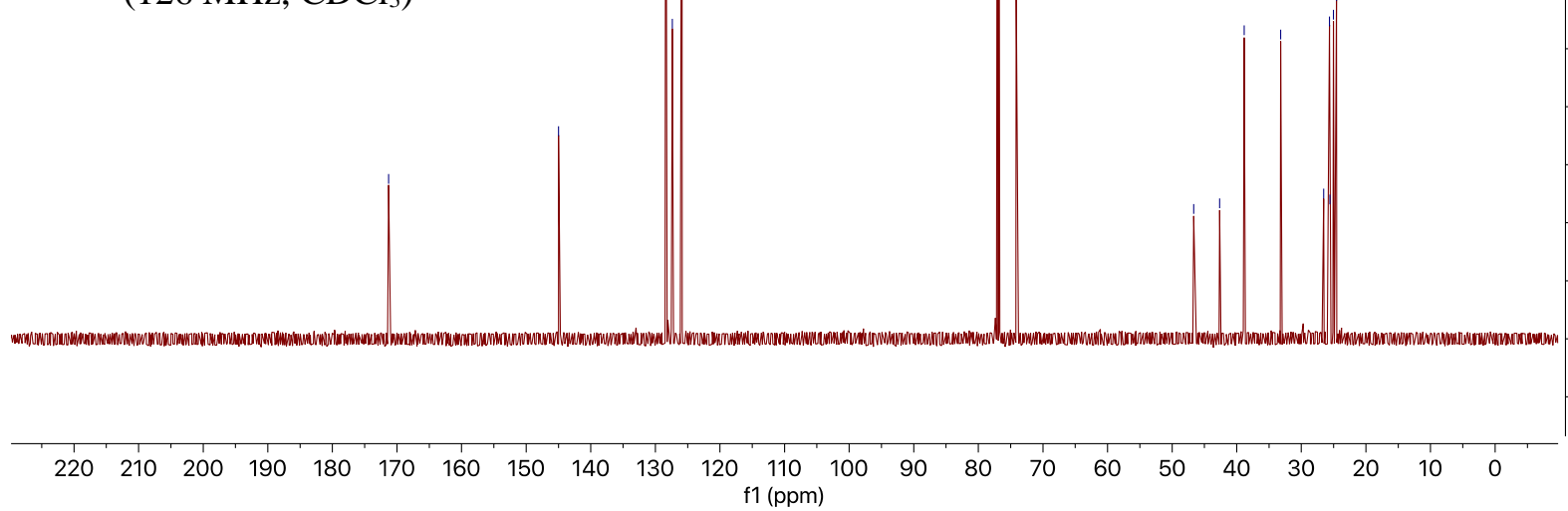




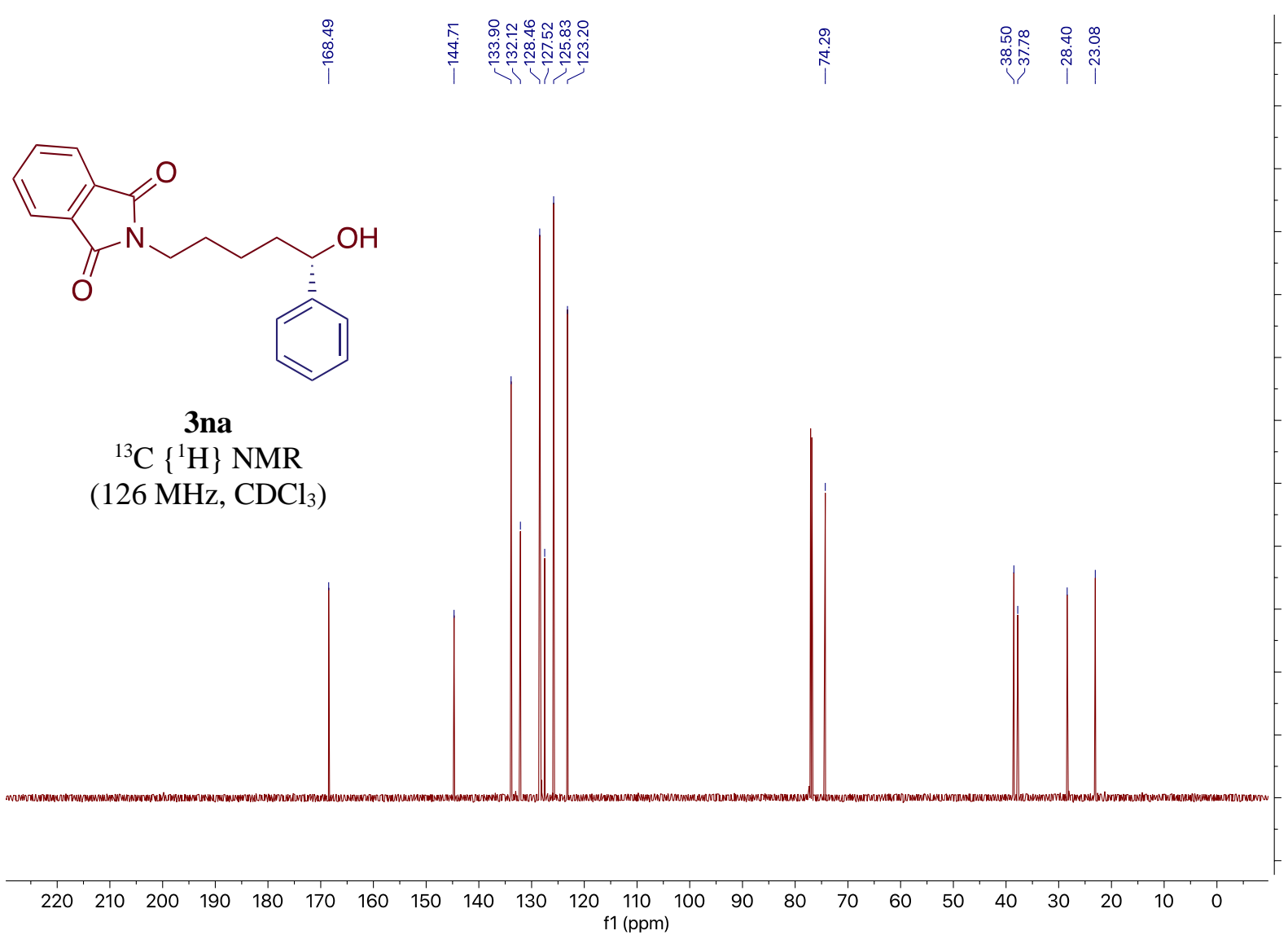




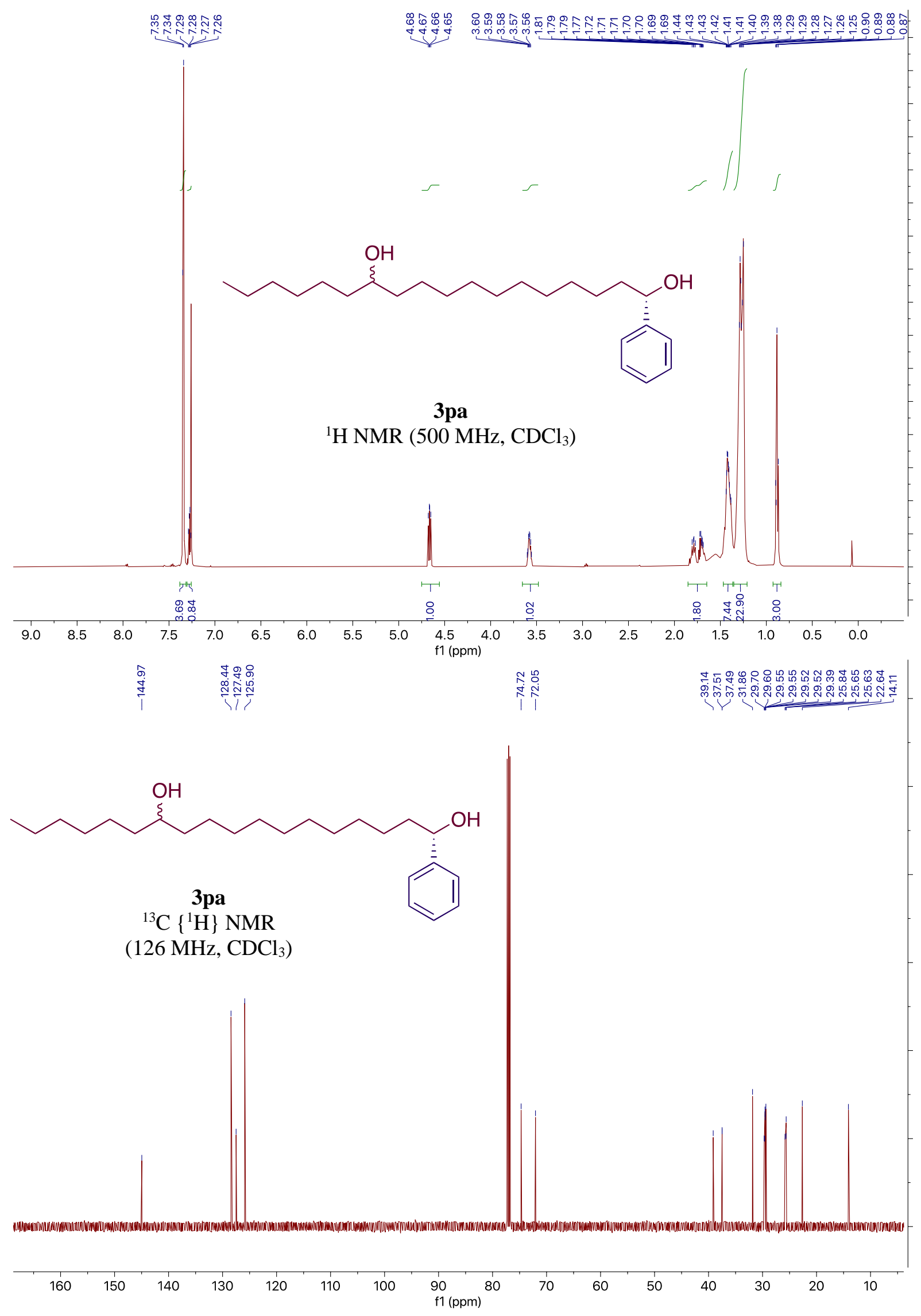



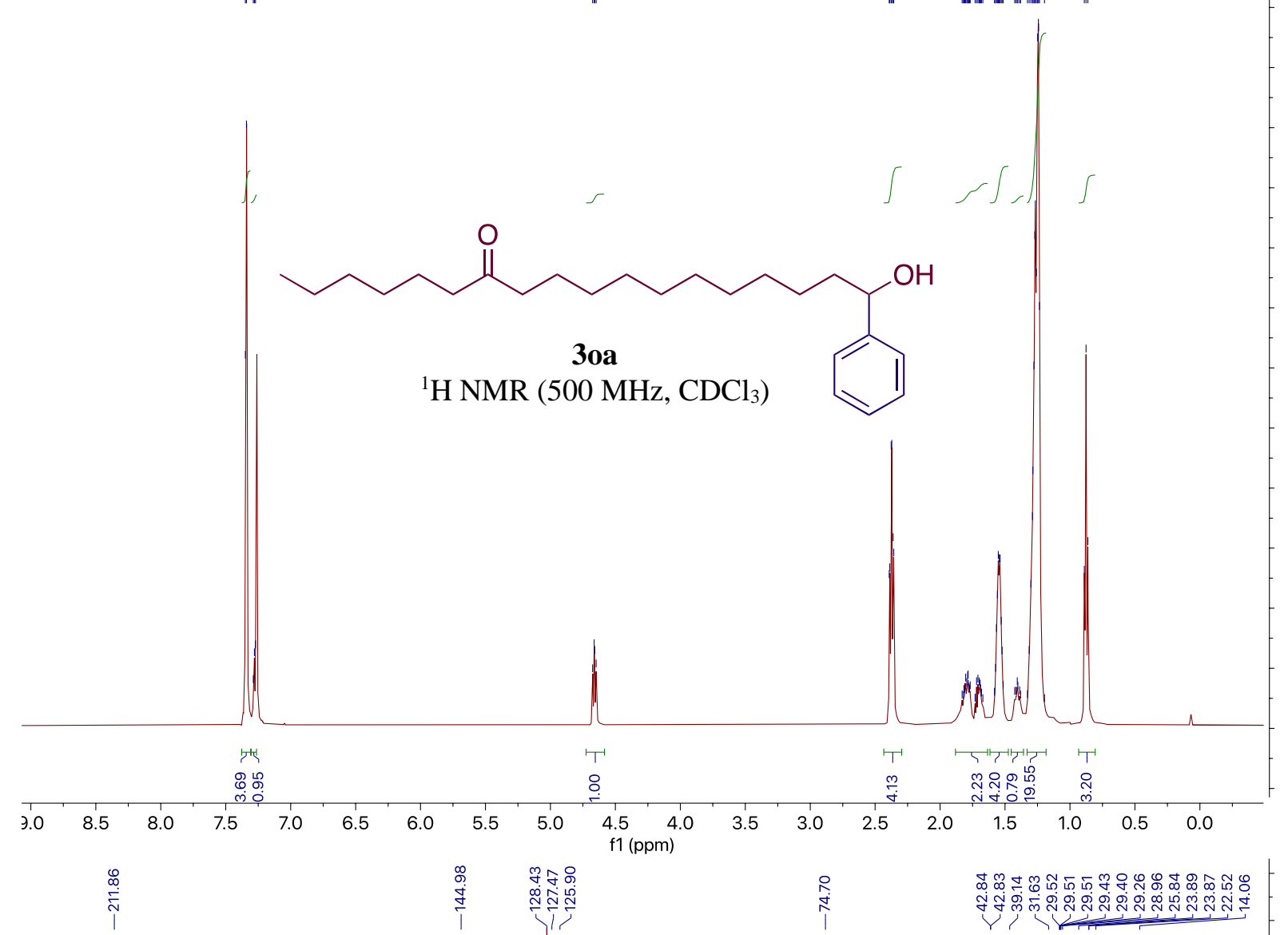

$\stackrel{\substack{i \\ \stackrel{i}{i}}}{2}$

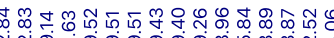

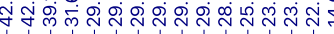<smiles>CCCCCCC(=O)CCCCCC</smiles>

3oa

${ }^{13} \mathrm{C}\left\{{ }^{1} \mathrm{H}\right\}$ NMR $\left(126 \mathrm{MHz}, \mathrm{CDCl}_{3}\right)$

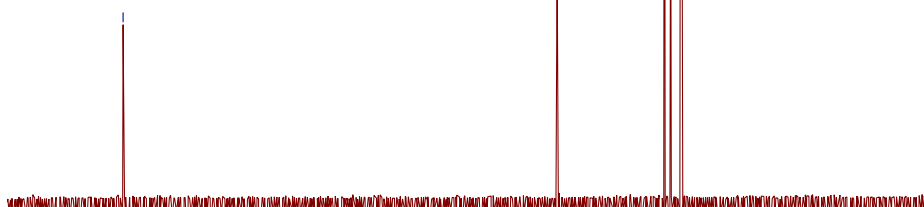

紊突<smiles>CCCCCCC(O)c1ccccc1</smiles>

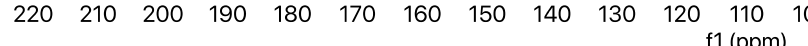



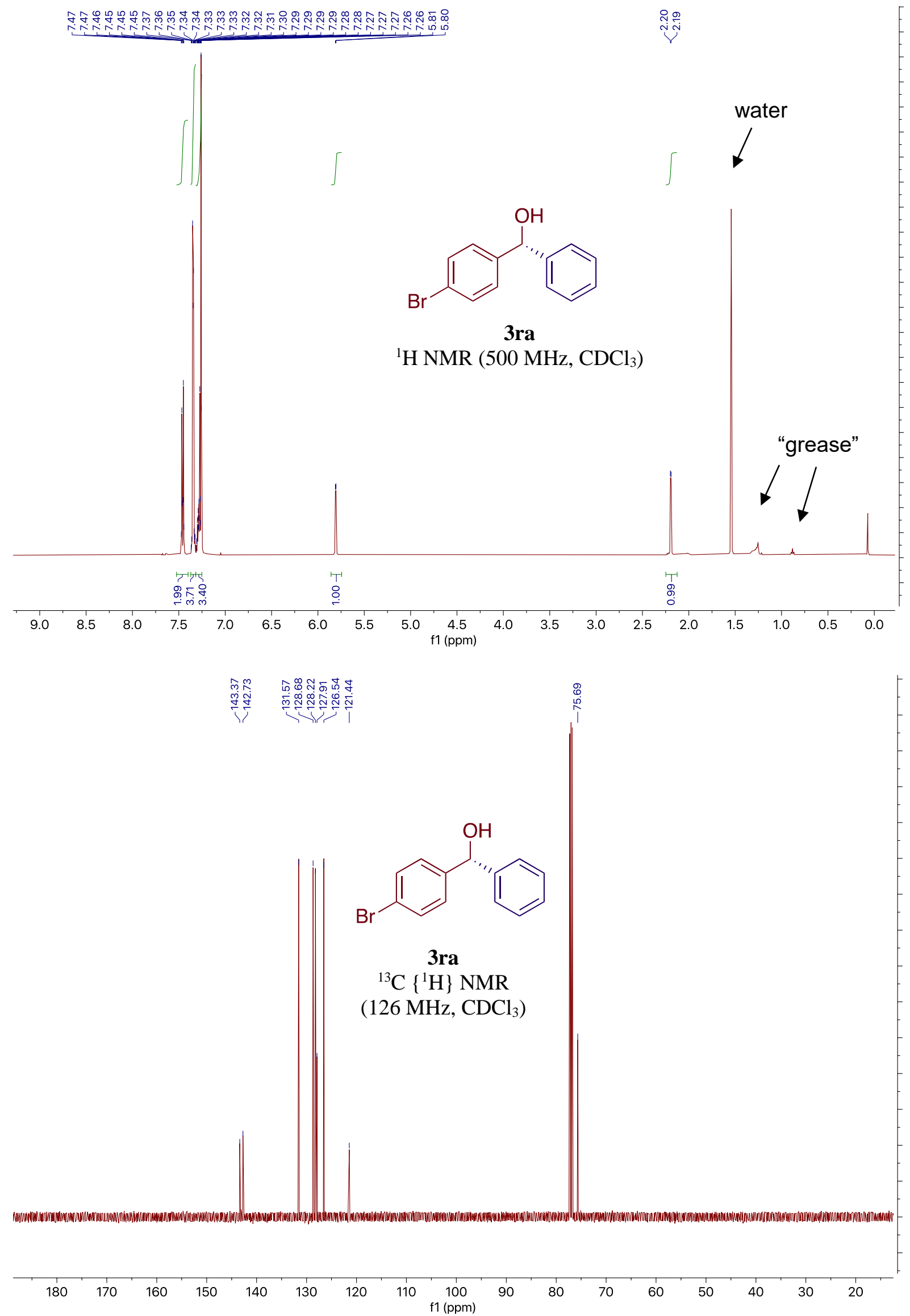


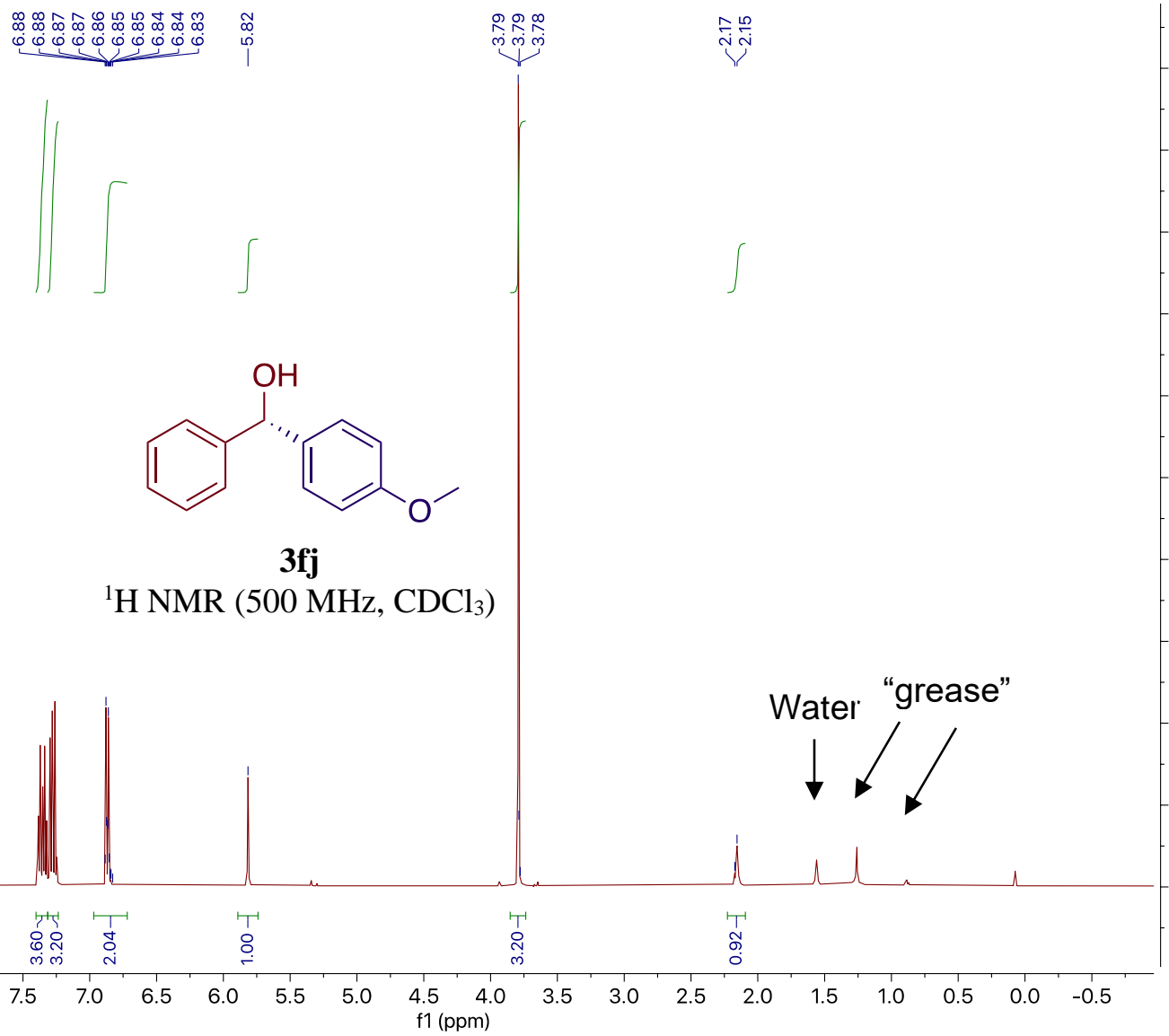

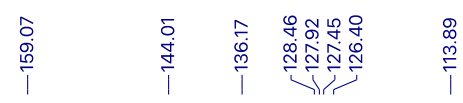

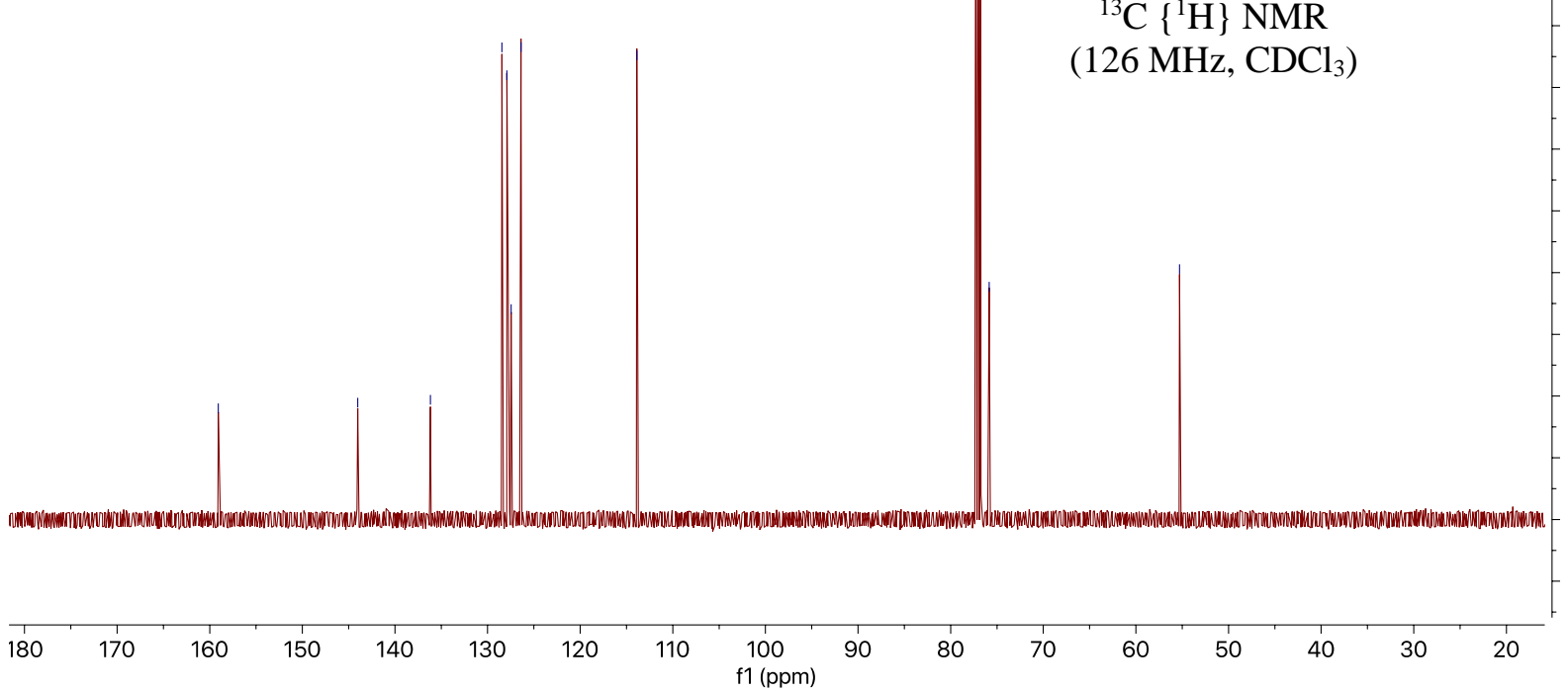




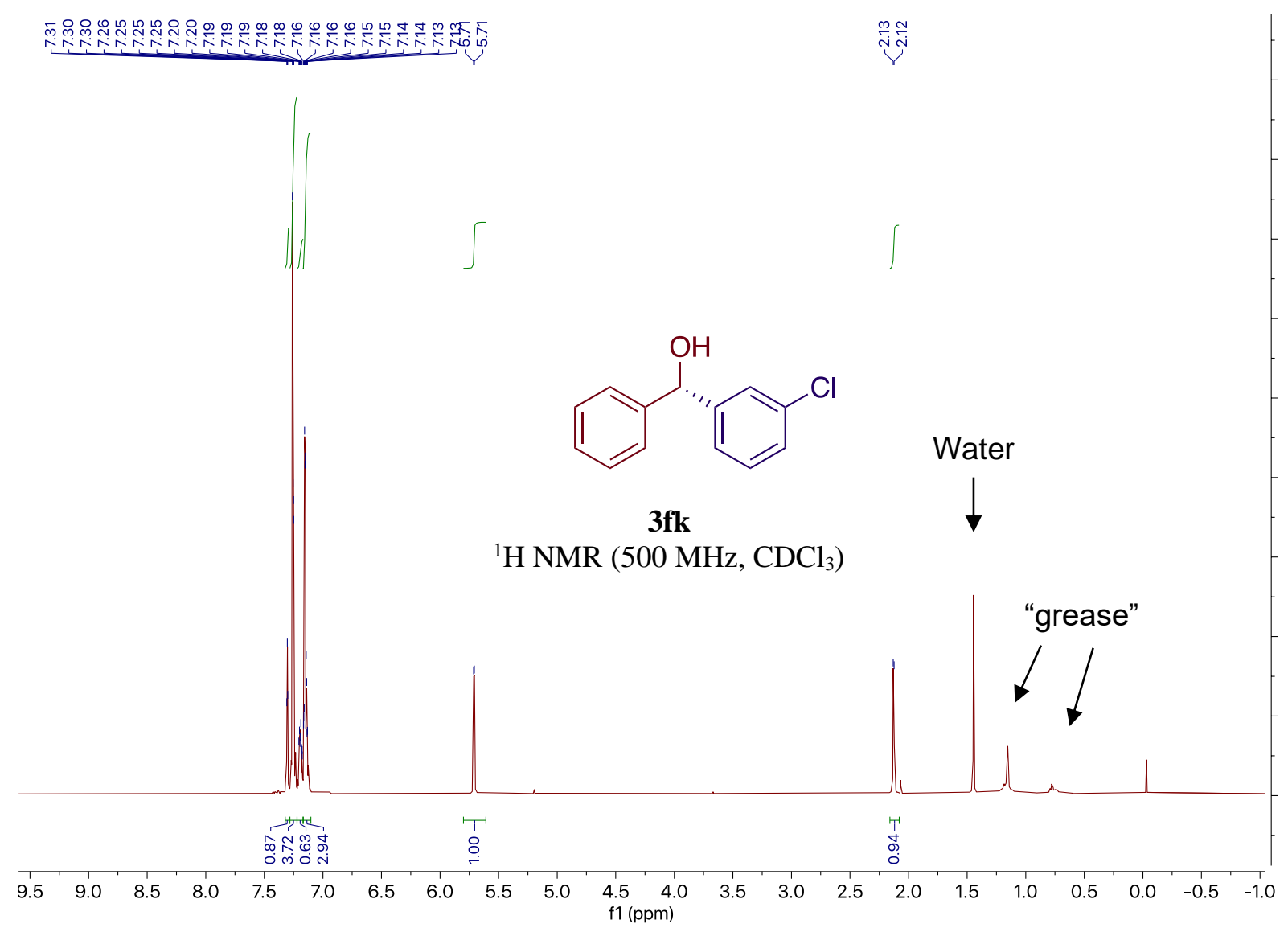

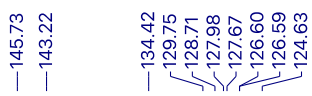<smiles>OC(c1ccccc1)c1cccc(Cl)c1</smiles>

3fk

${ }^{13} \mathrm{C}\left\{{ }^{1} \mathrm{H}\right\}$ NMR

(126 MHz, $\mathrm{CDCl}_{3}$ )

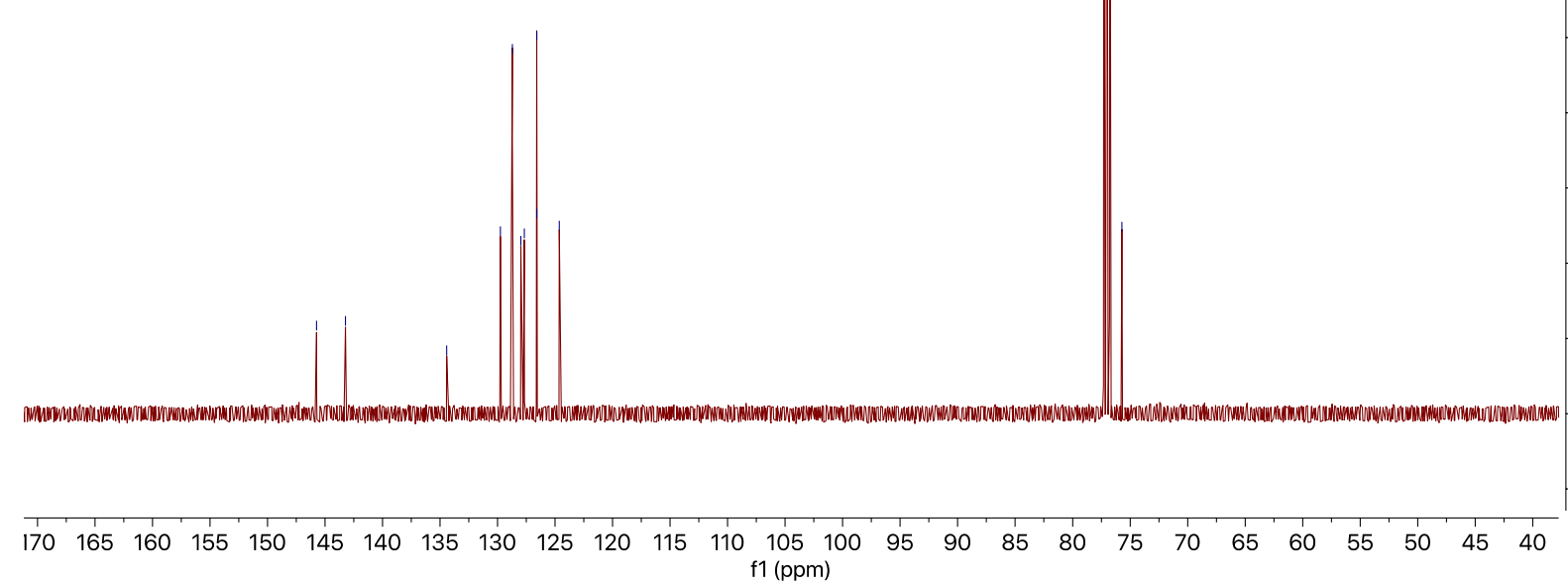



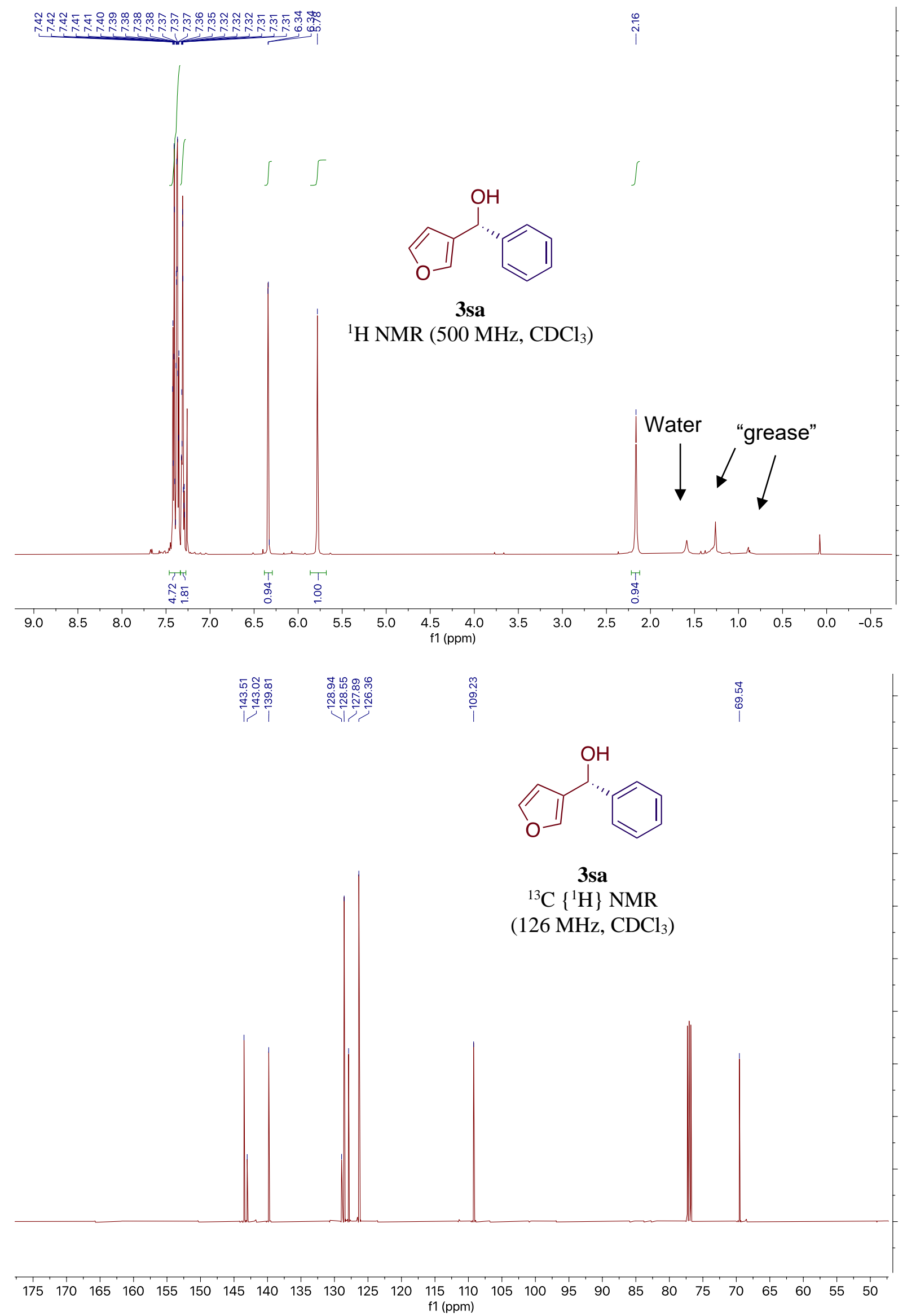

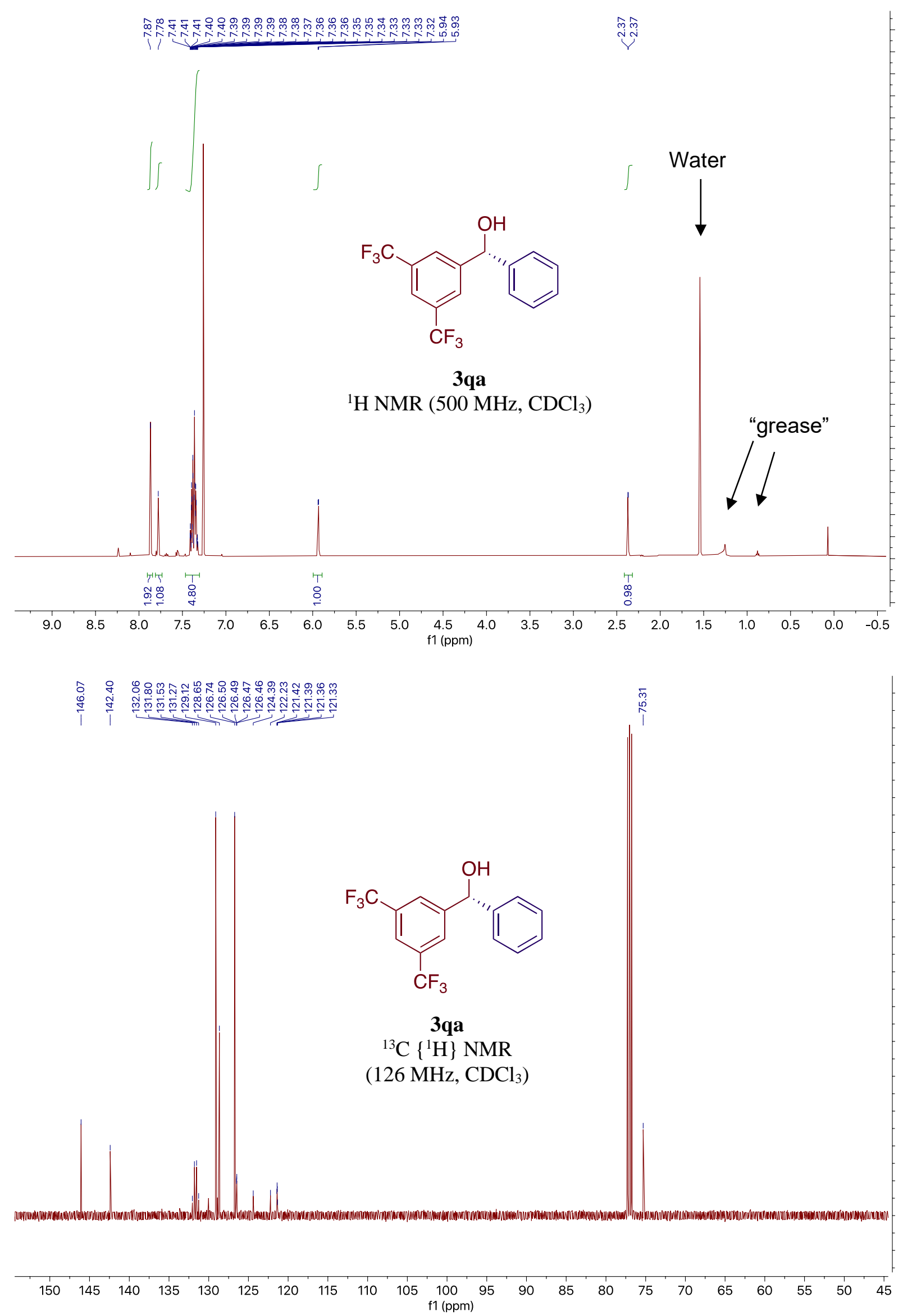
<smiles>OC(c1ccccc1)c1cc(C(F)(F)F)cc(C(F)(F)F)c1</smiles>

3qa

${ }^{19} \mathrm{~F}$ NMR

(476 MHz, $\mathrm{CDCl}_{3}$ )

\begin{tabular}{rlllllllllllllllllllllllllllllllll}
\hline 5 & 0 & -5 & -10 & -15 & -20 & -25 & -30 & -35 & -40 & -45 & -50 & -55 & -60 & -65 & -70 & -75 & -80 & -85 & -90 & -95 & -100 & -105 & -110 & -115
\end{tabular} 


\section{Chiral SFC Traces}

3aa
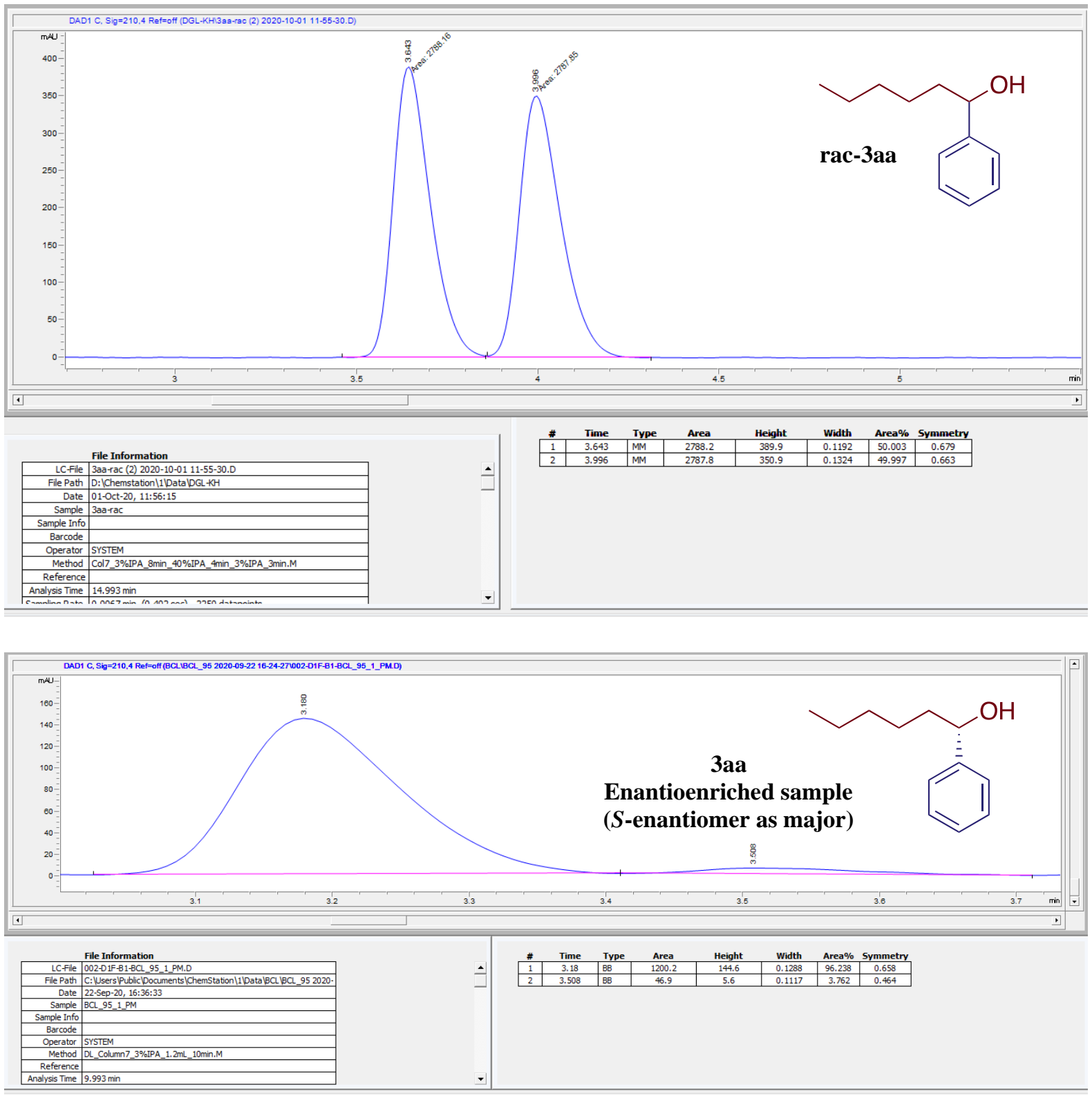
3aa - a bigger scale
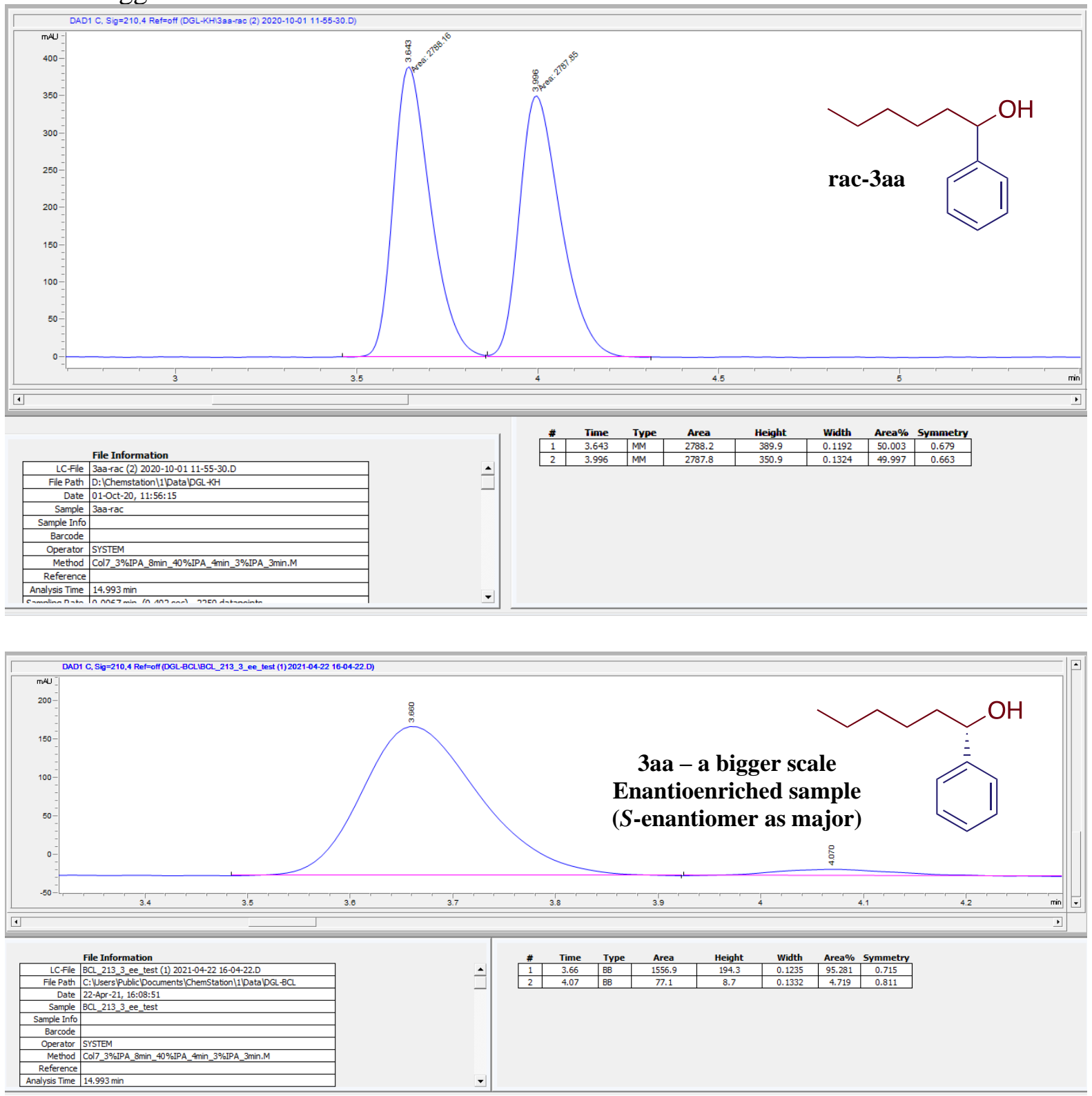
3ac
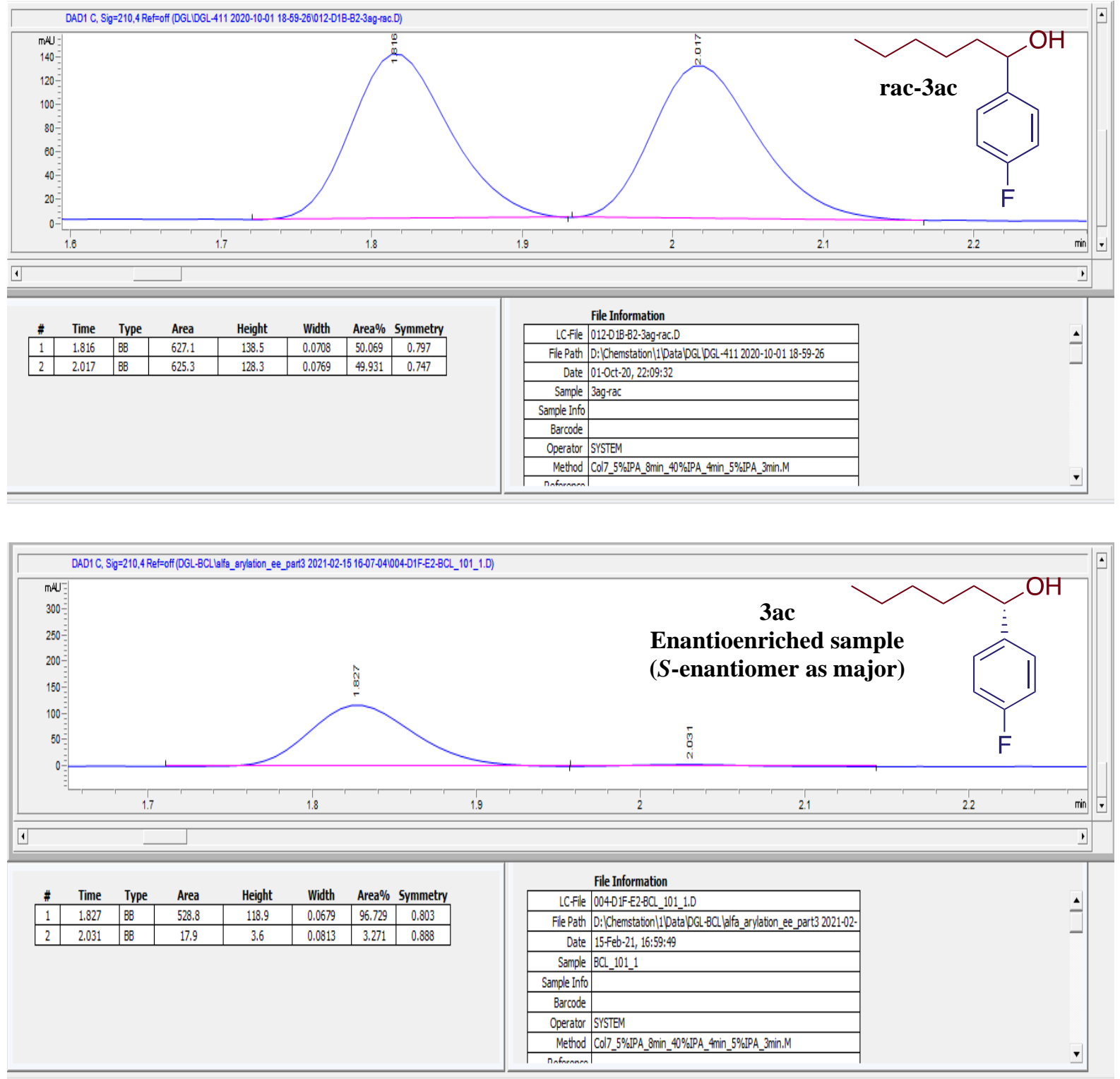
3ae
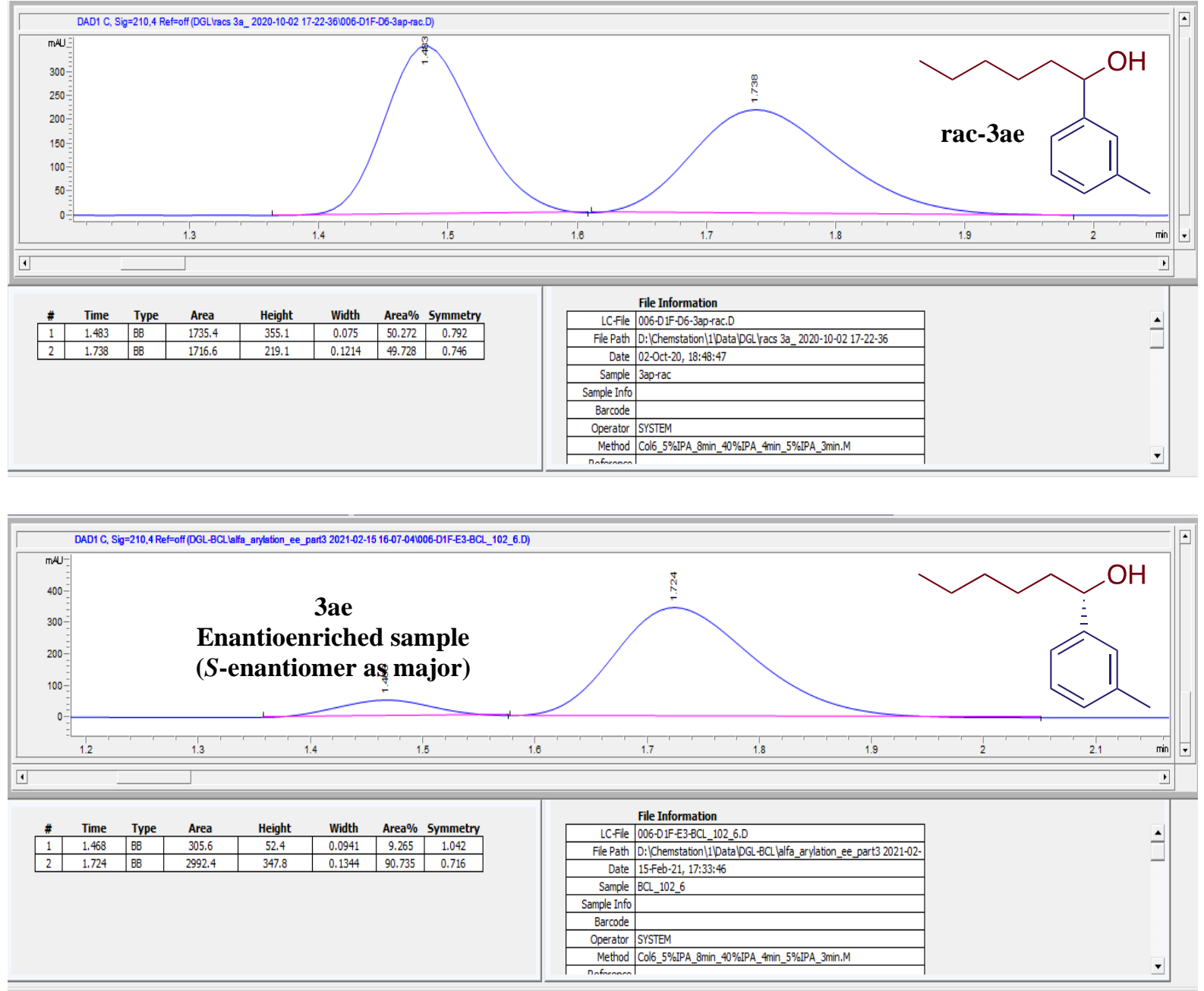
3ag
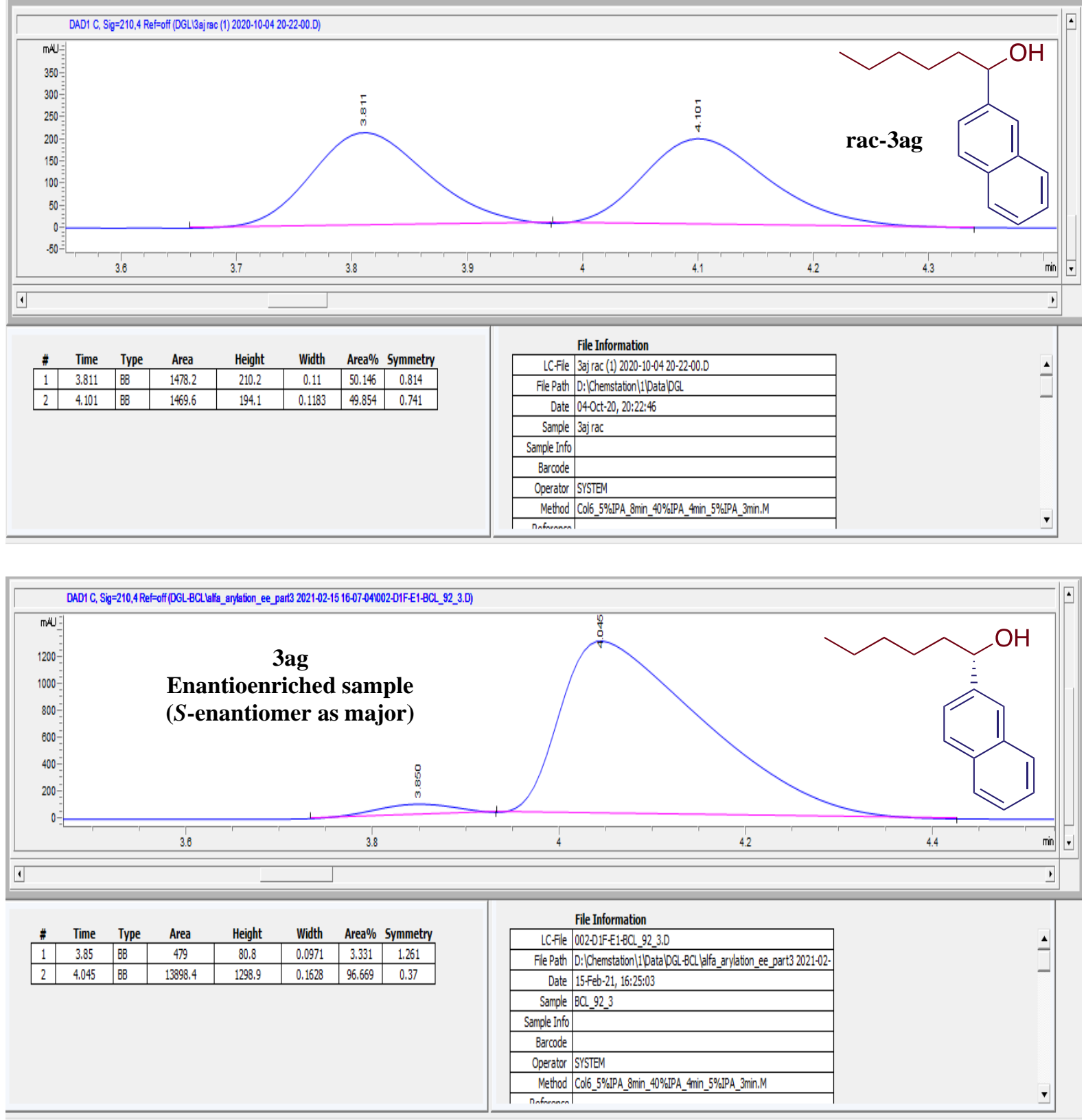
3ab
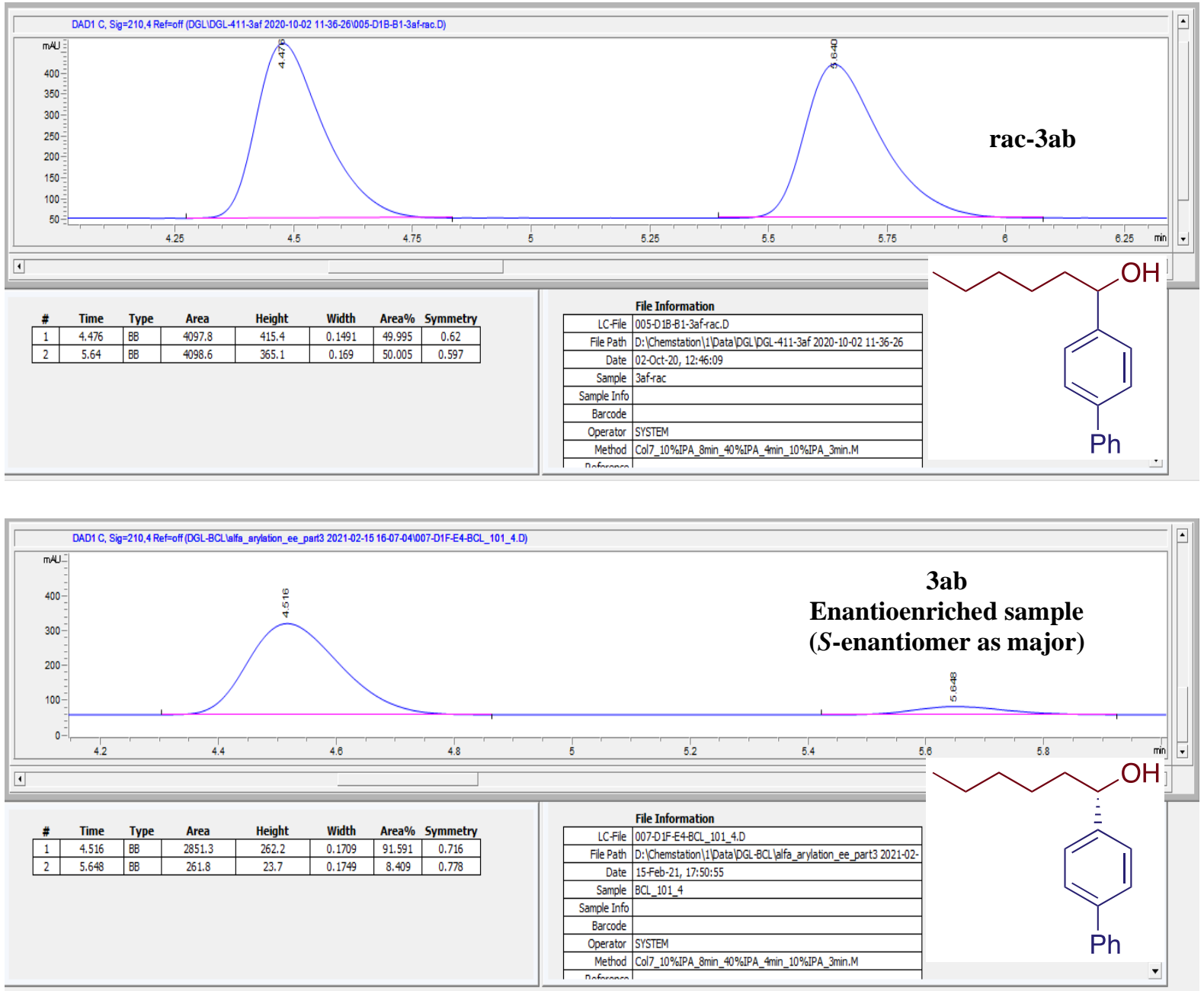
3ah
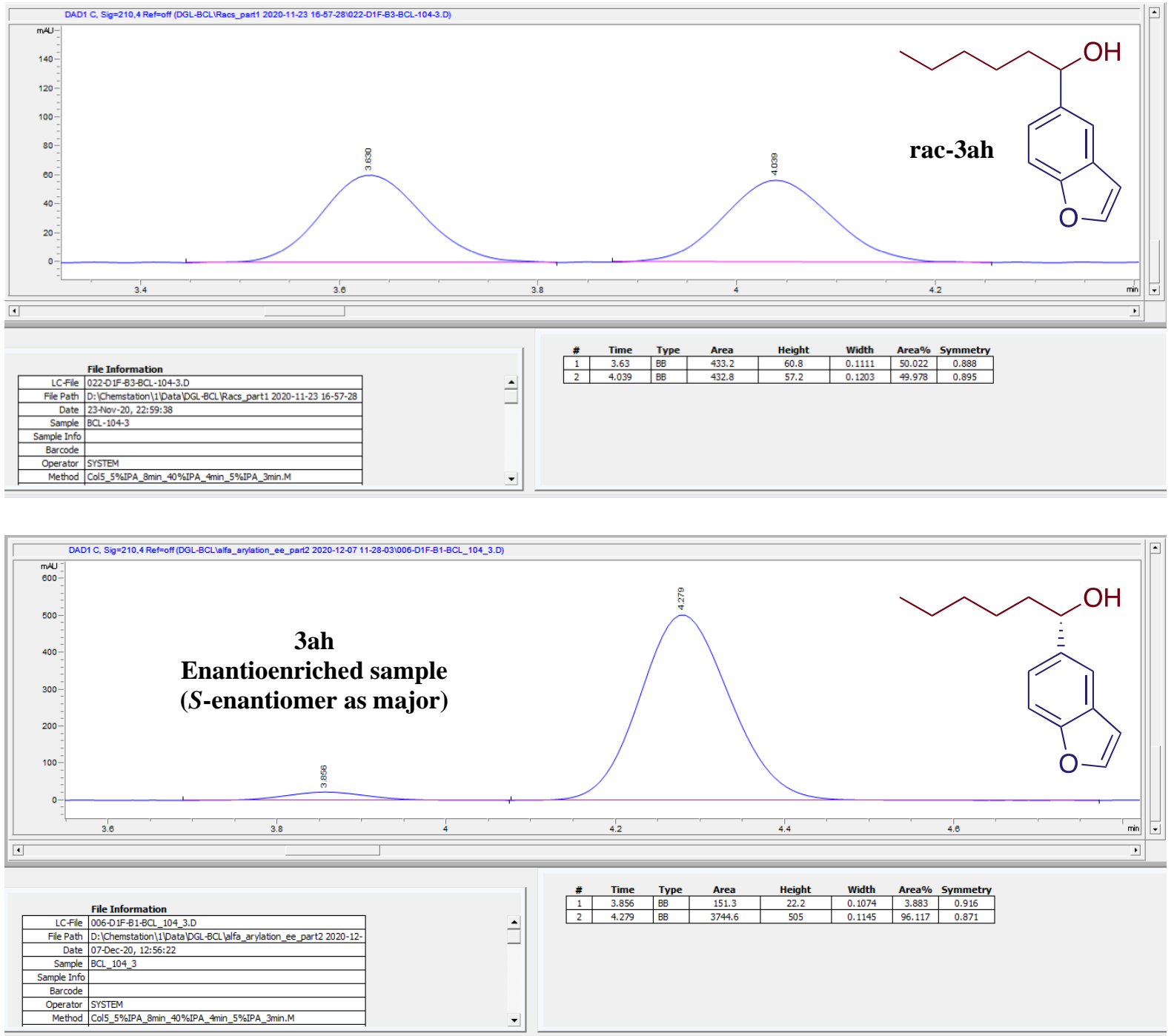
3ai
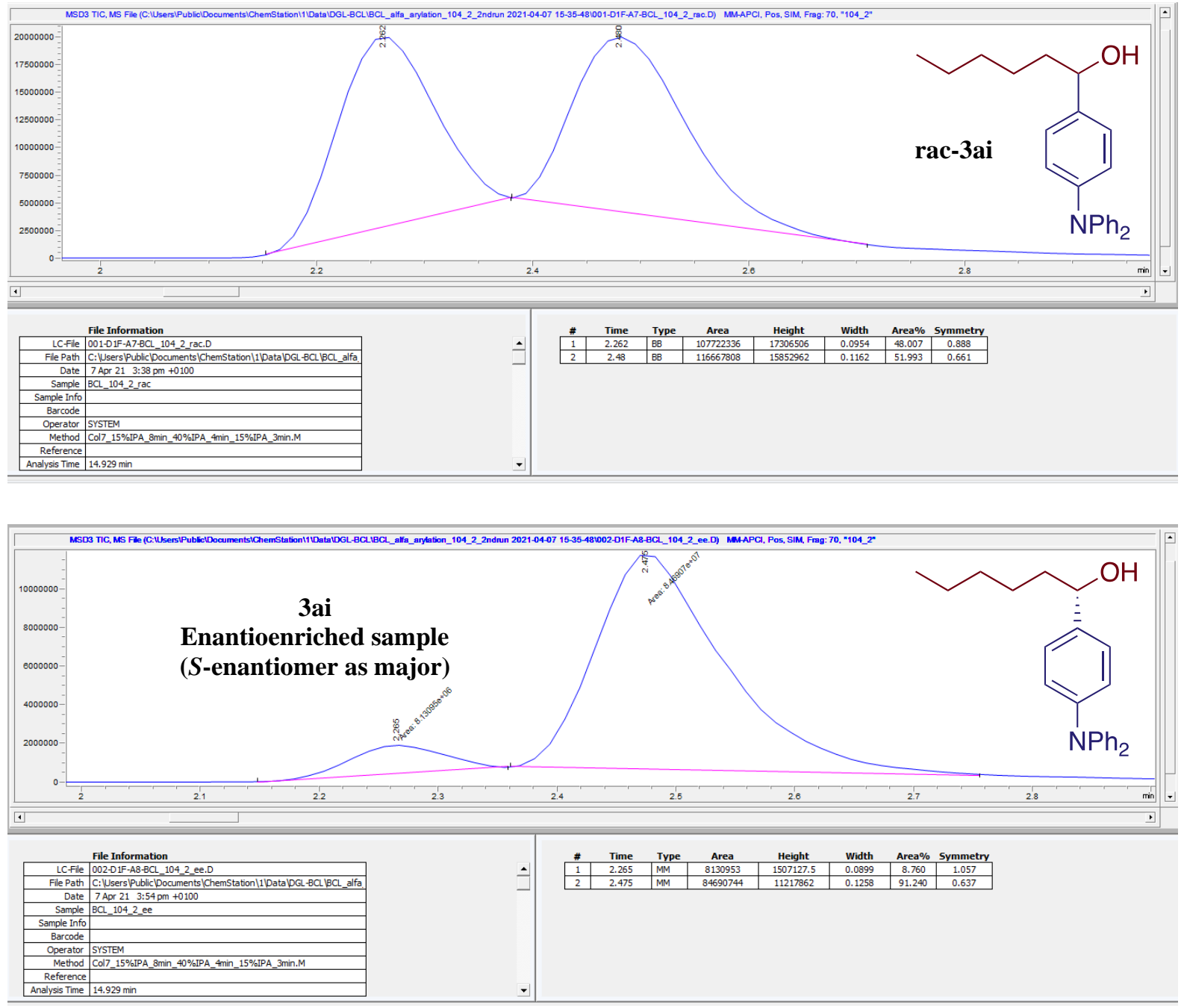


\section{3ga}
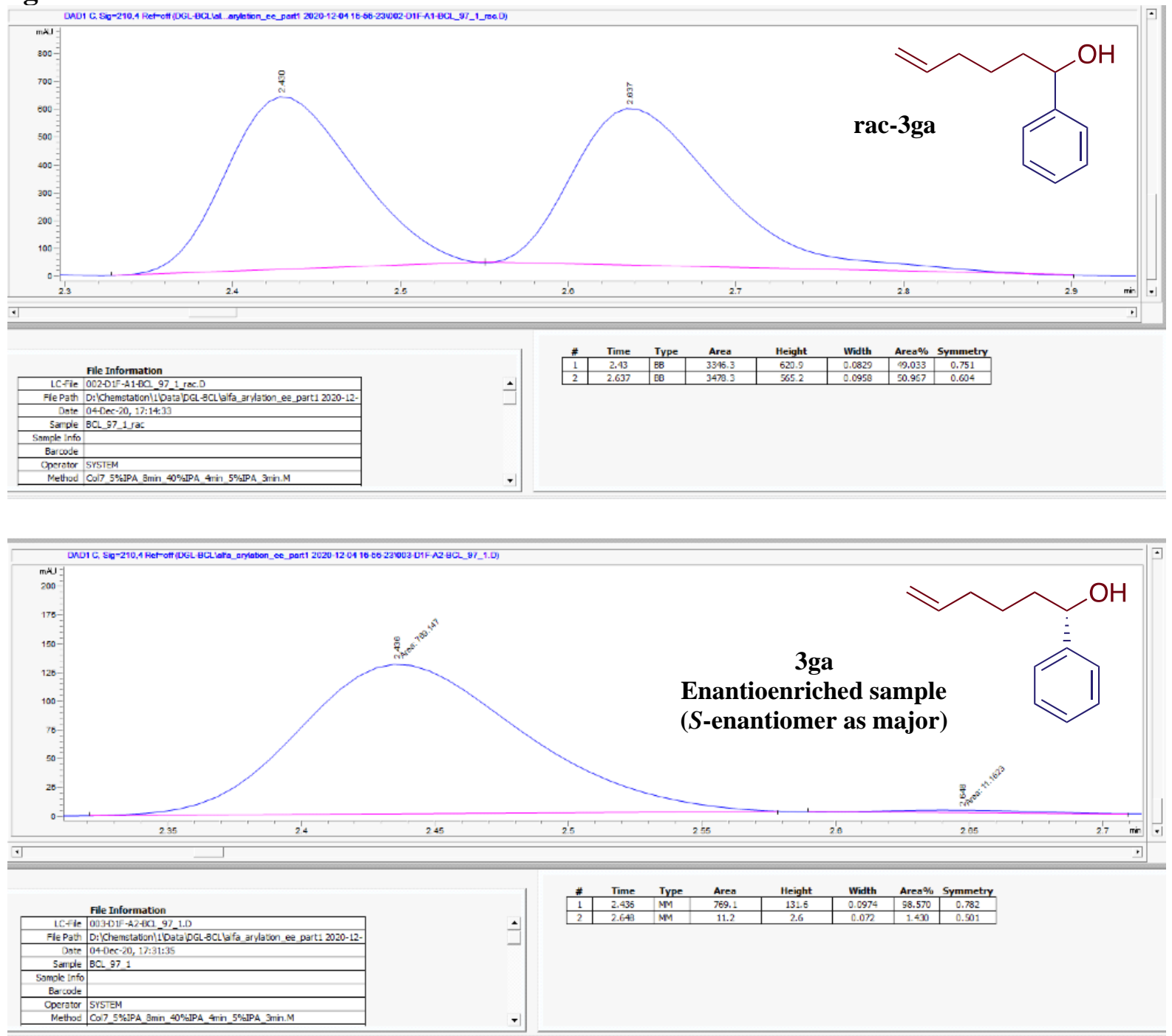
3ha
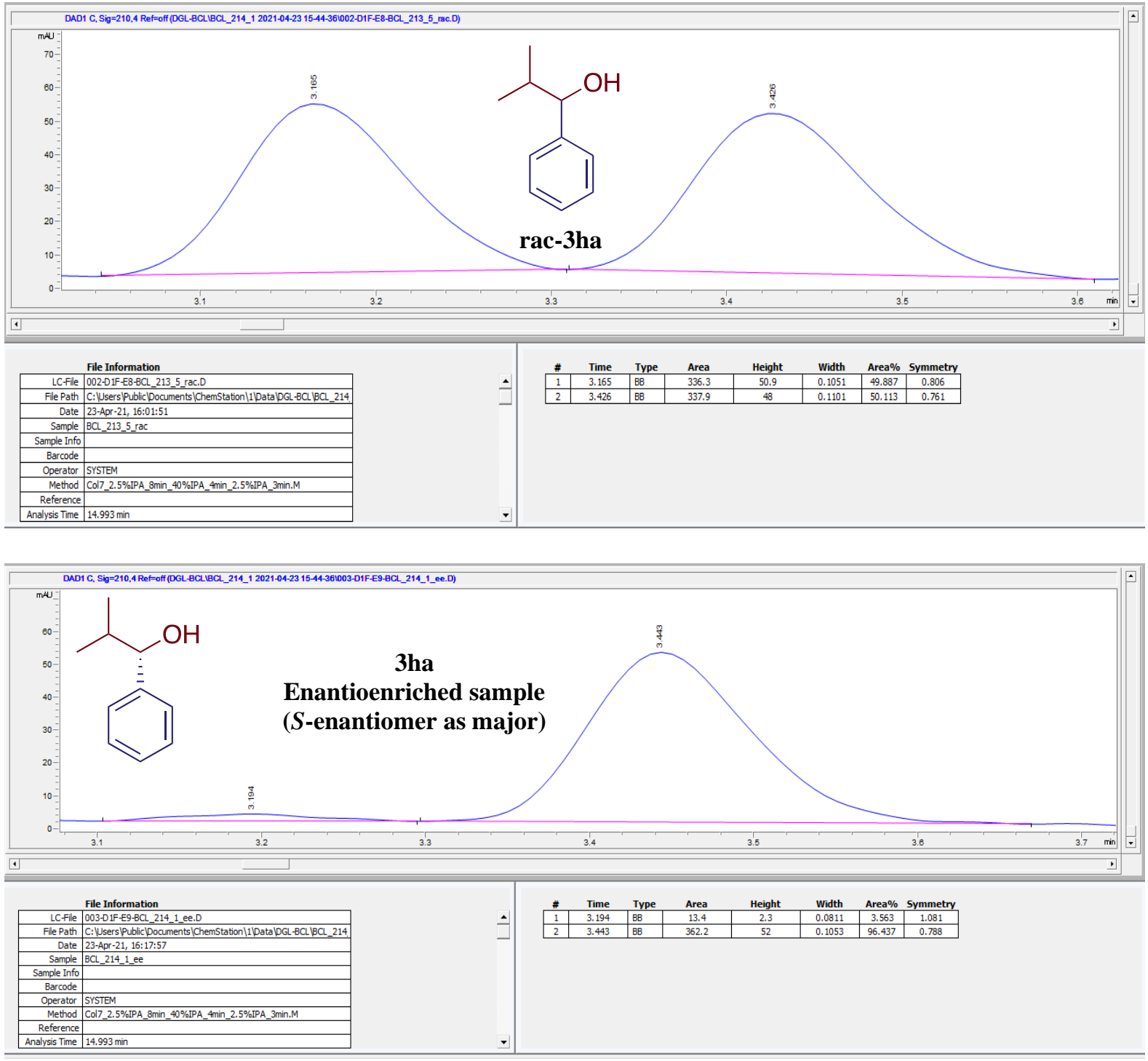
3ia
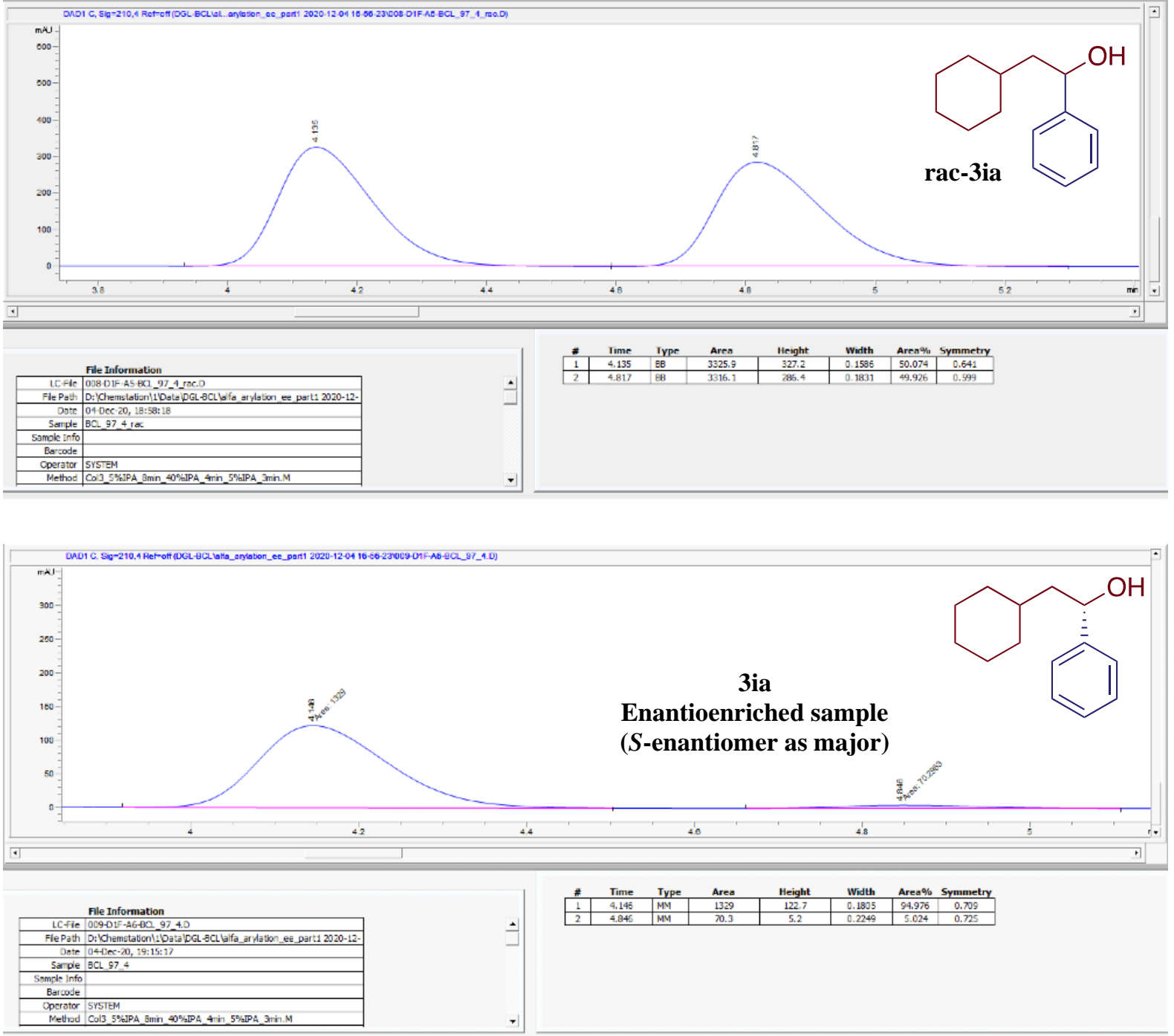


\section{3ja}

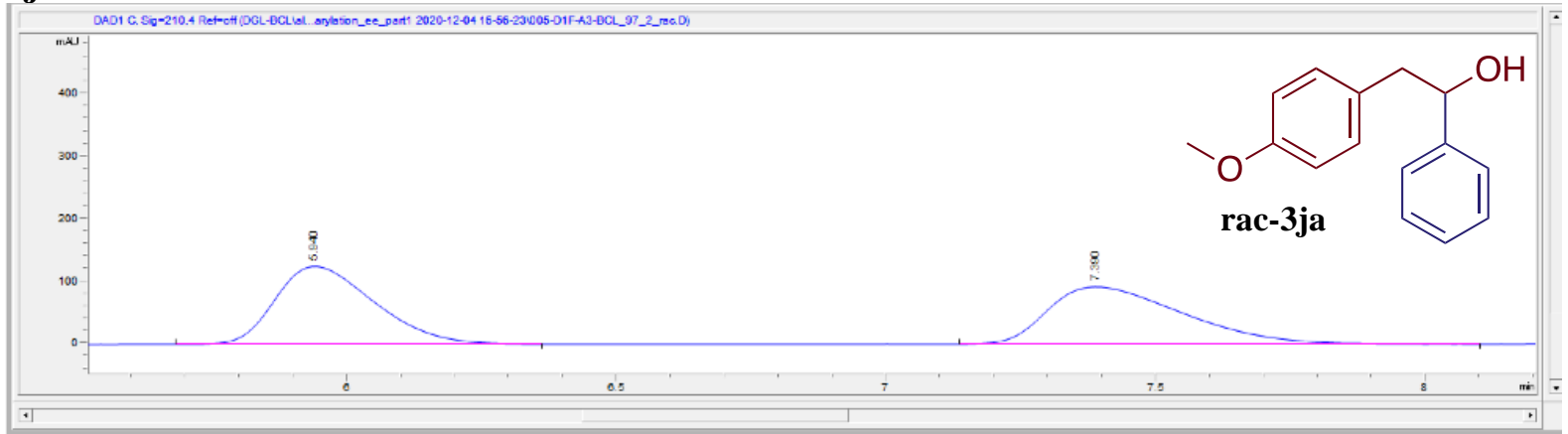

Filc Information

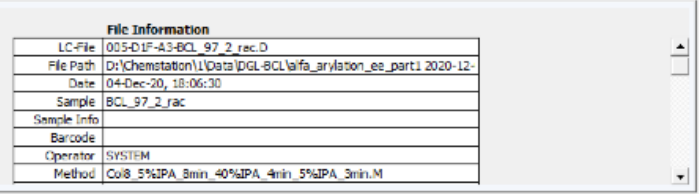

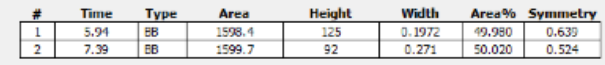

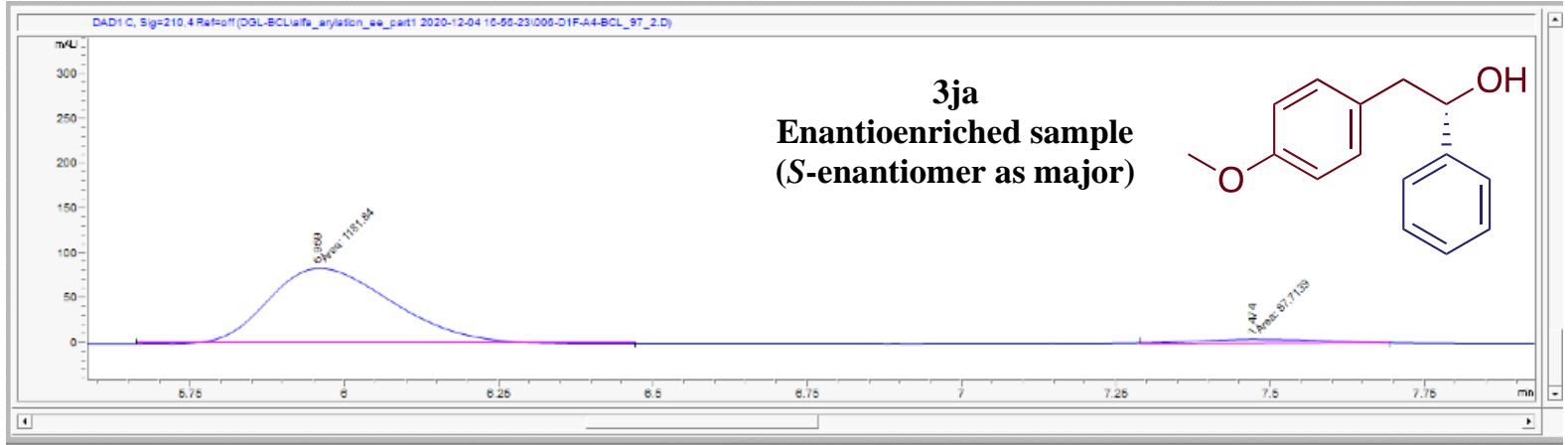

rile Intormation

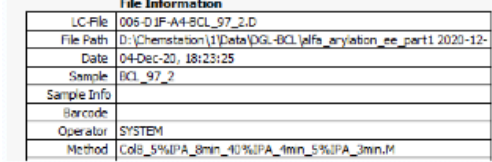

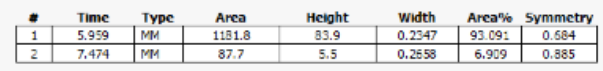


3ka
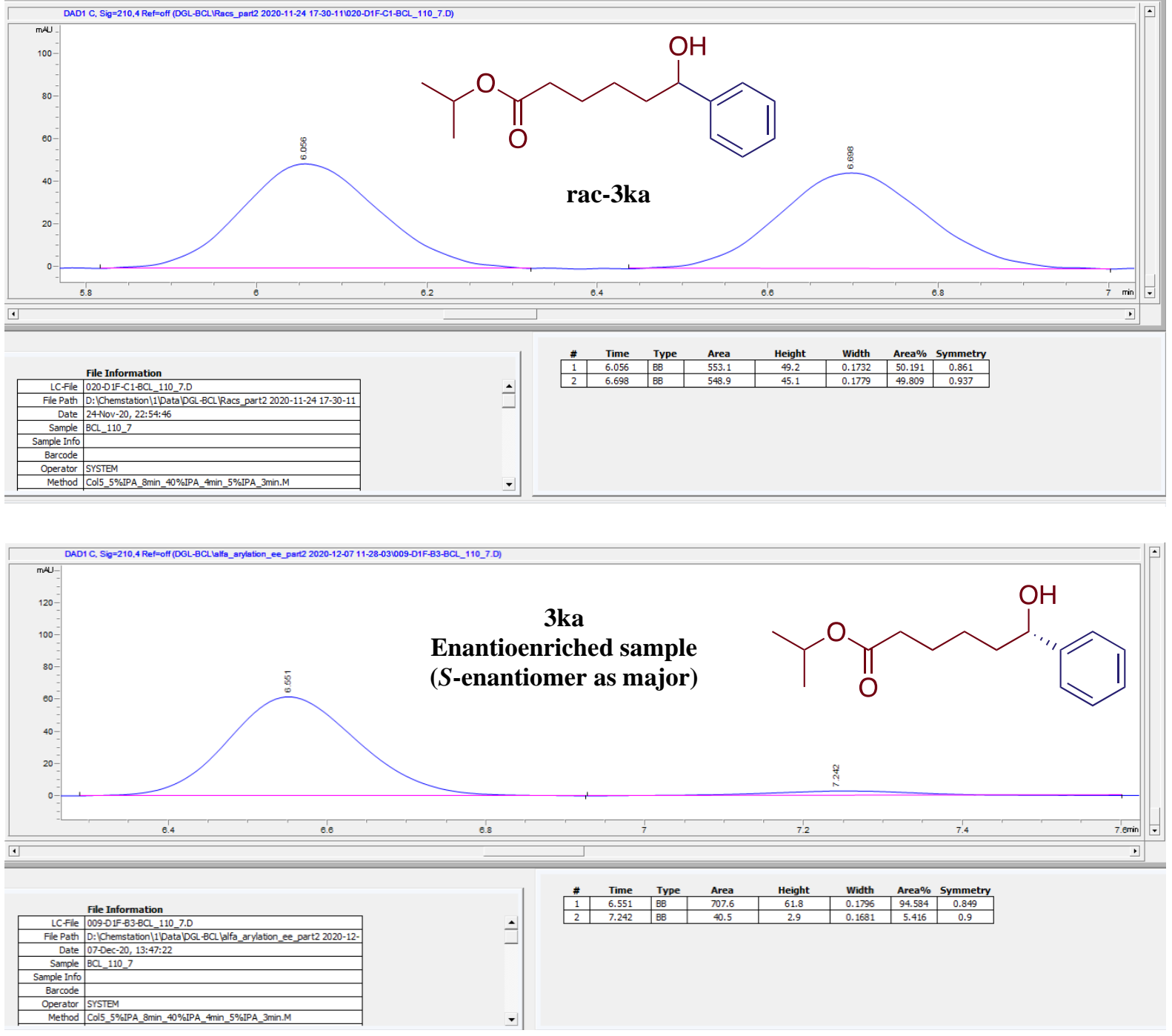
3ma
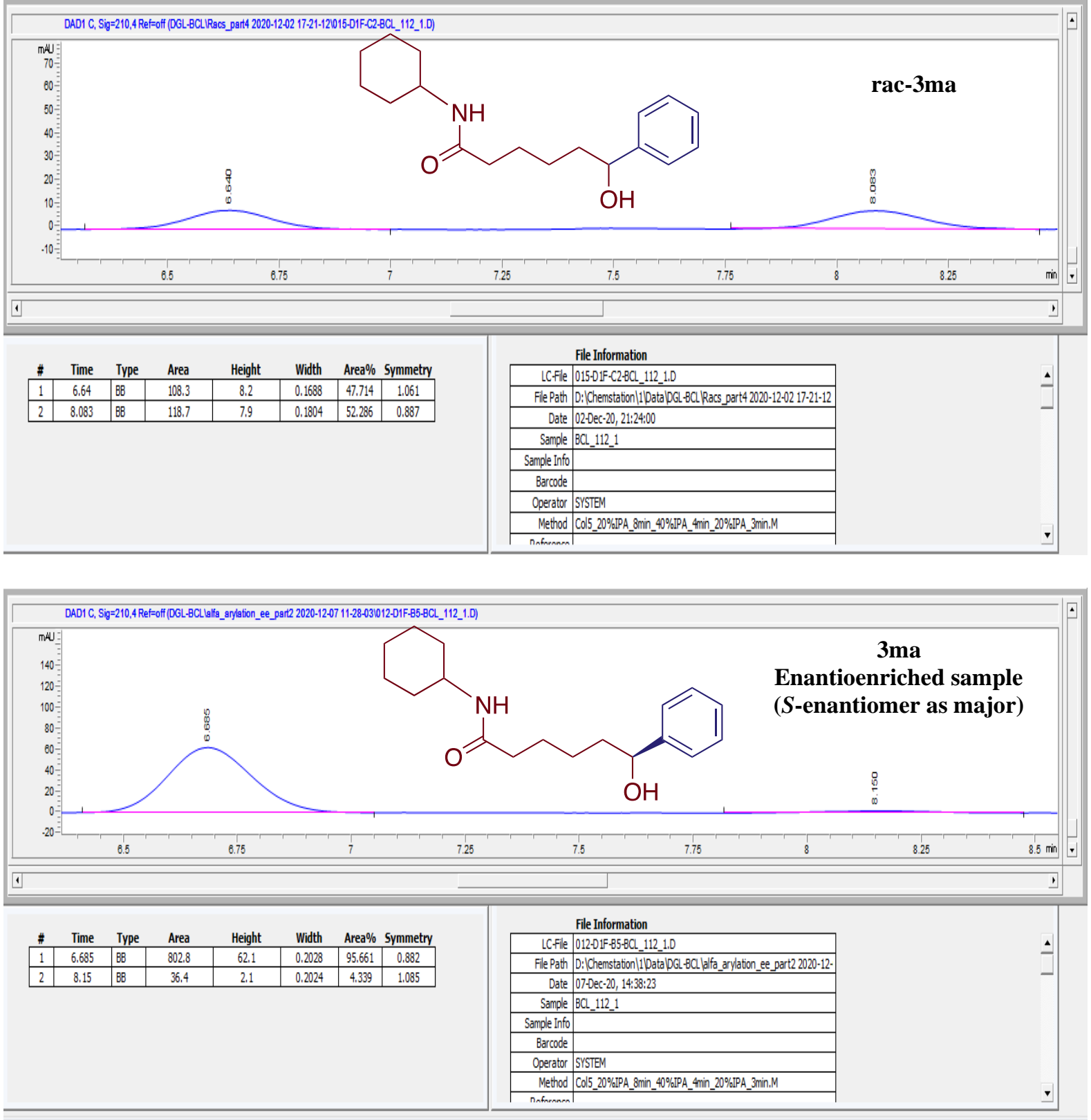
3la
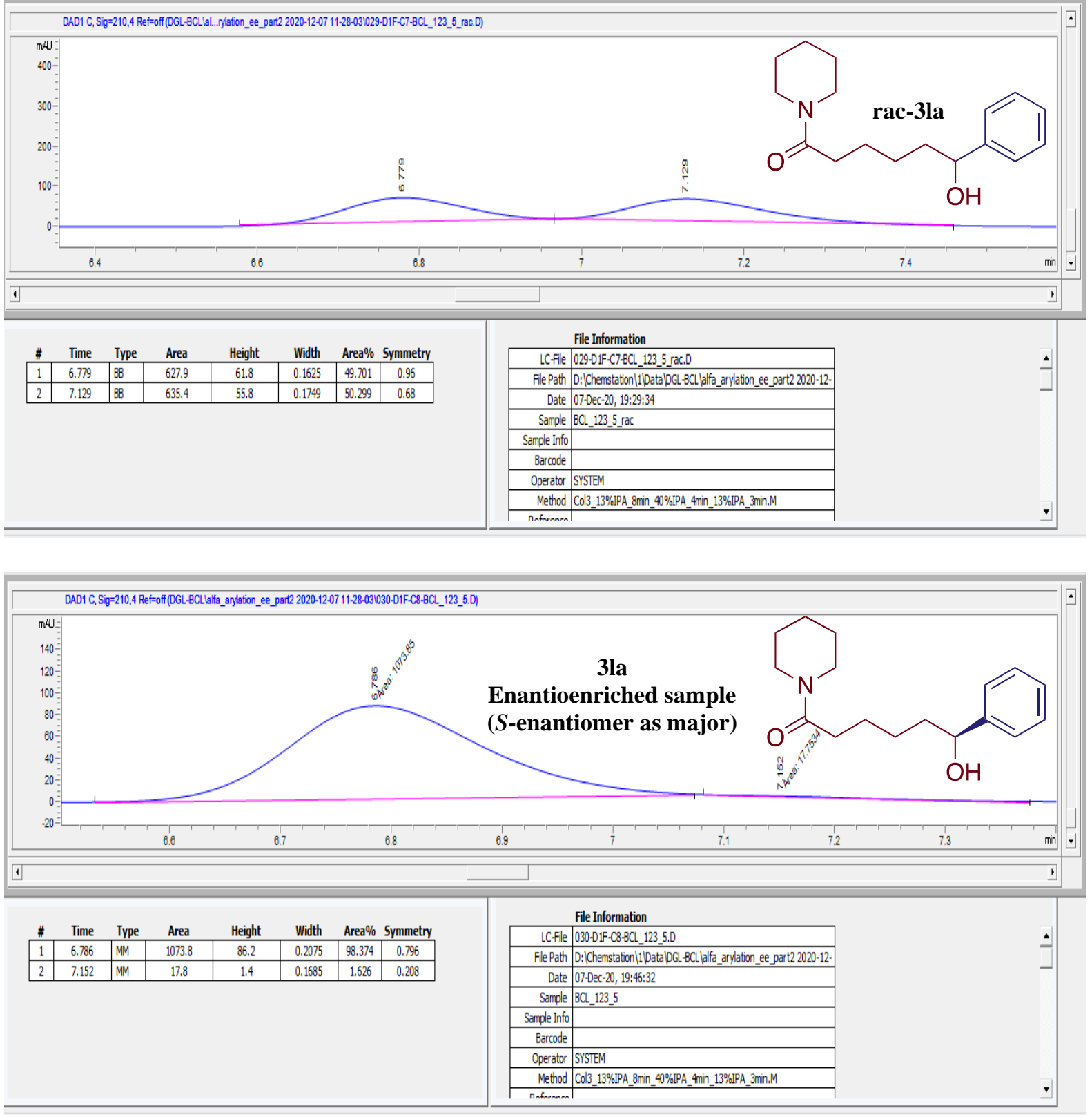
3na
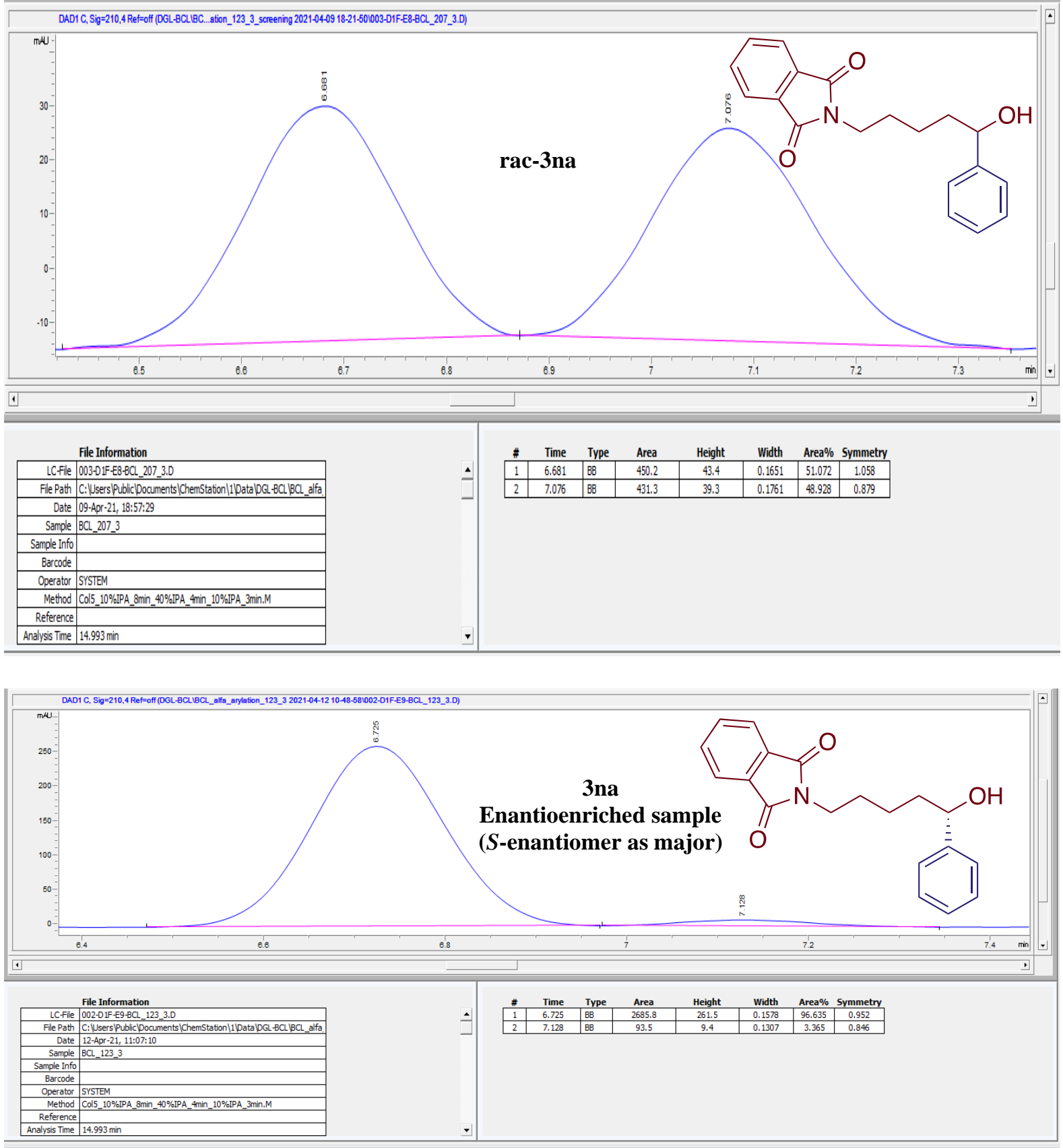
3pa'
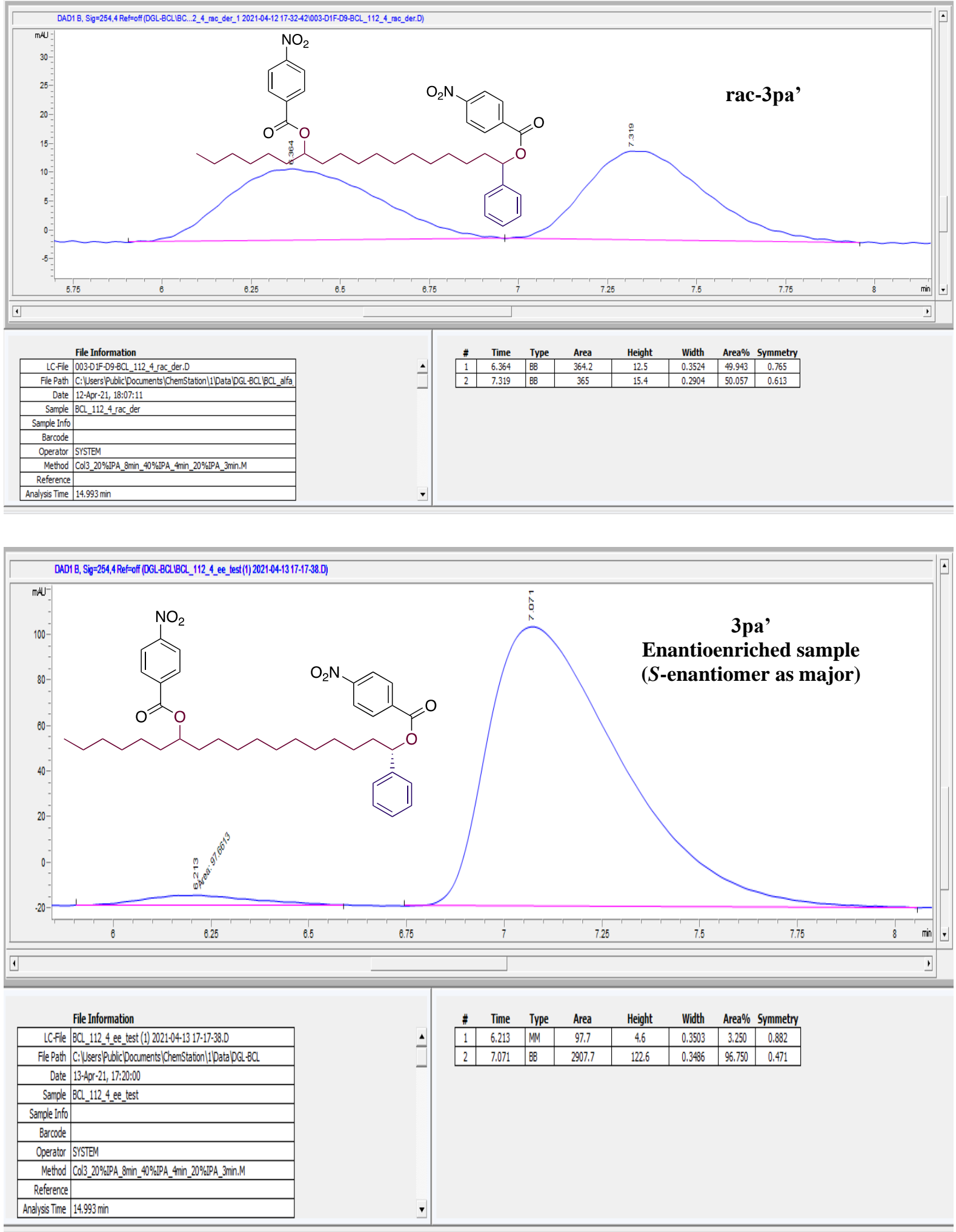
$30 a$
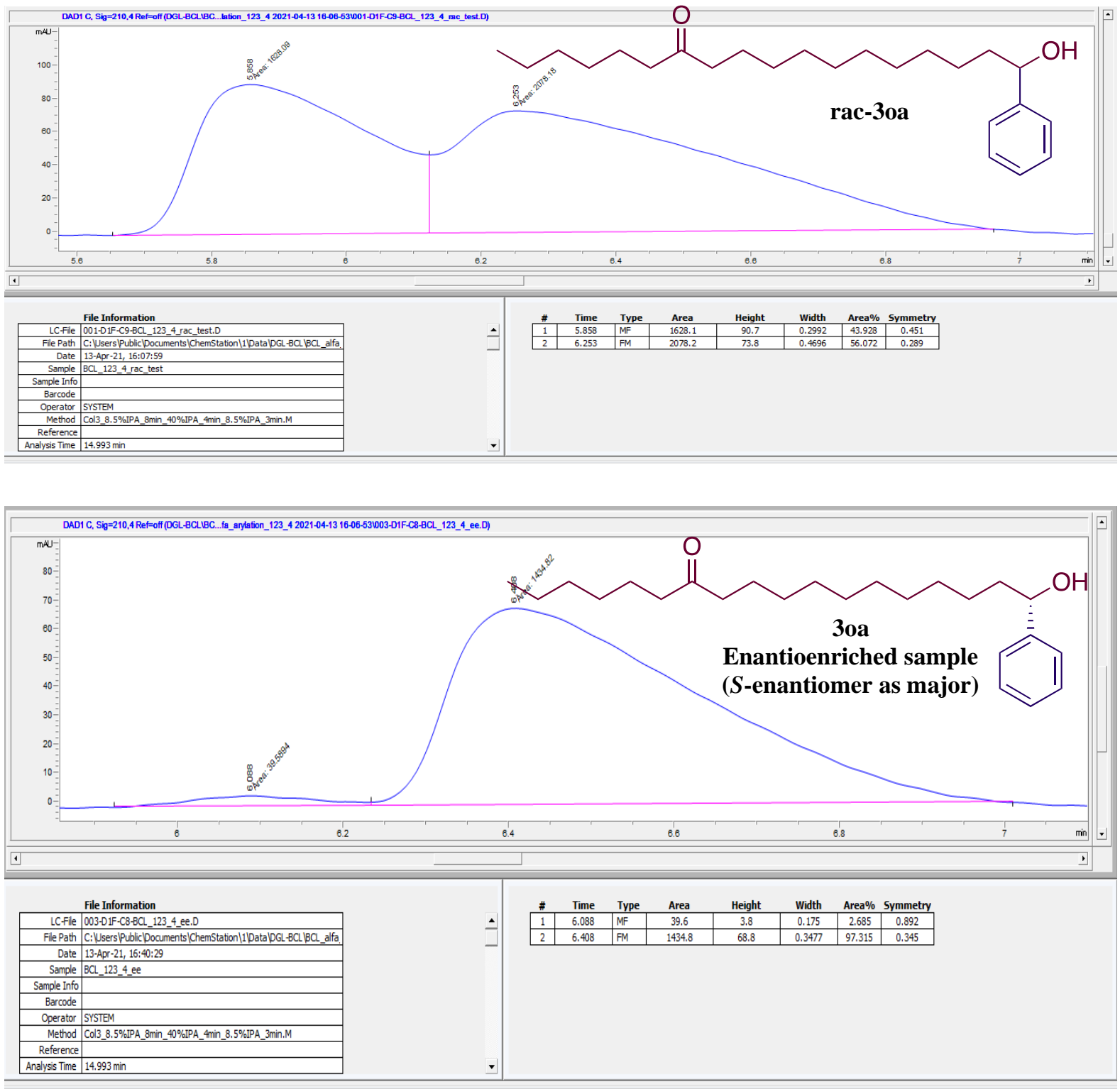
3ra
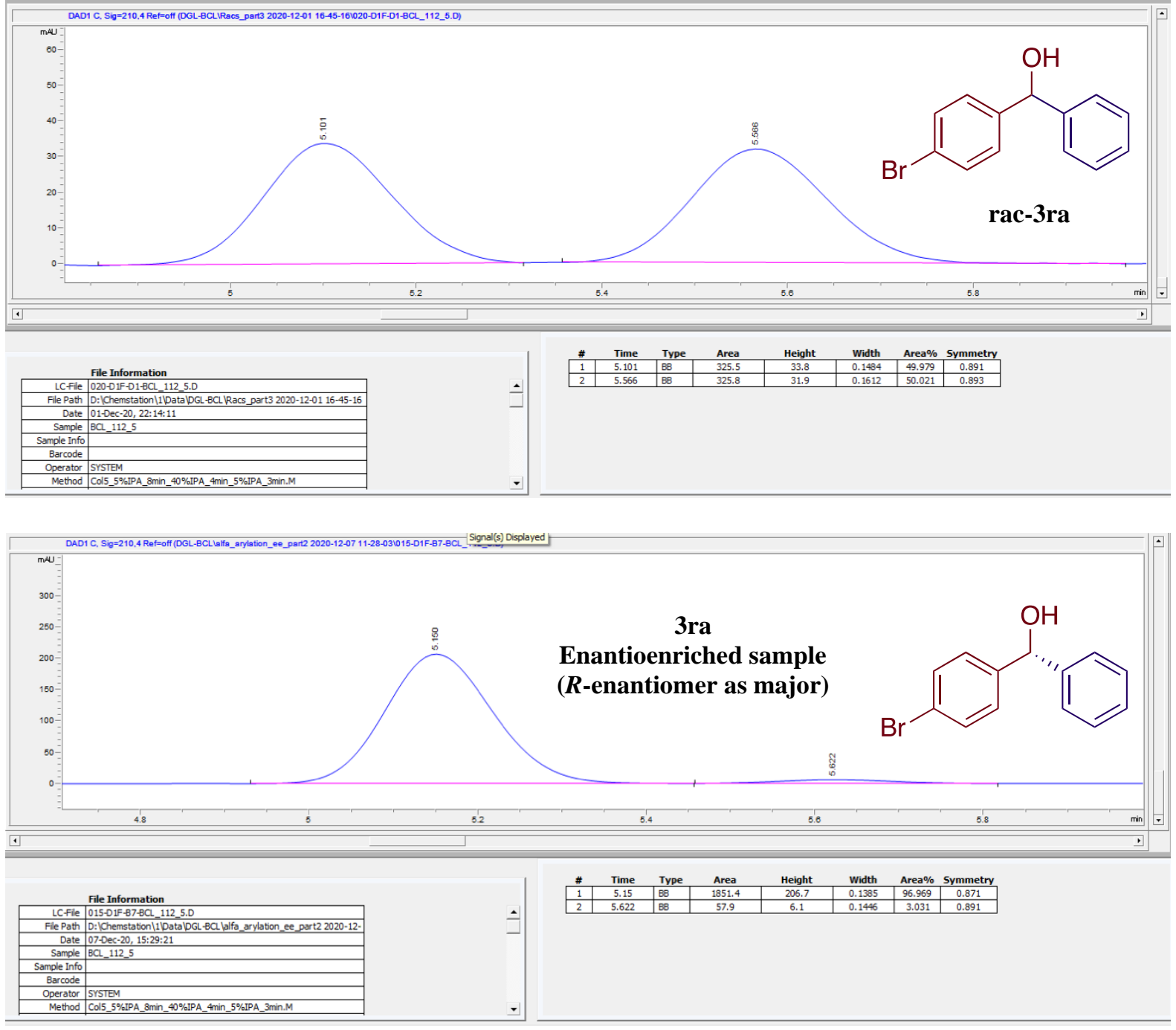
3fj
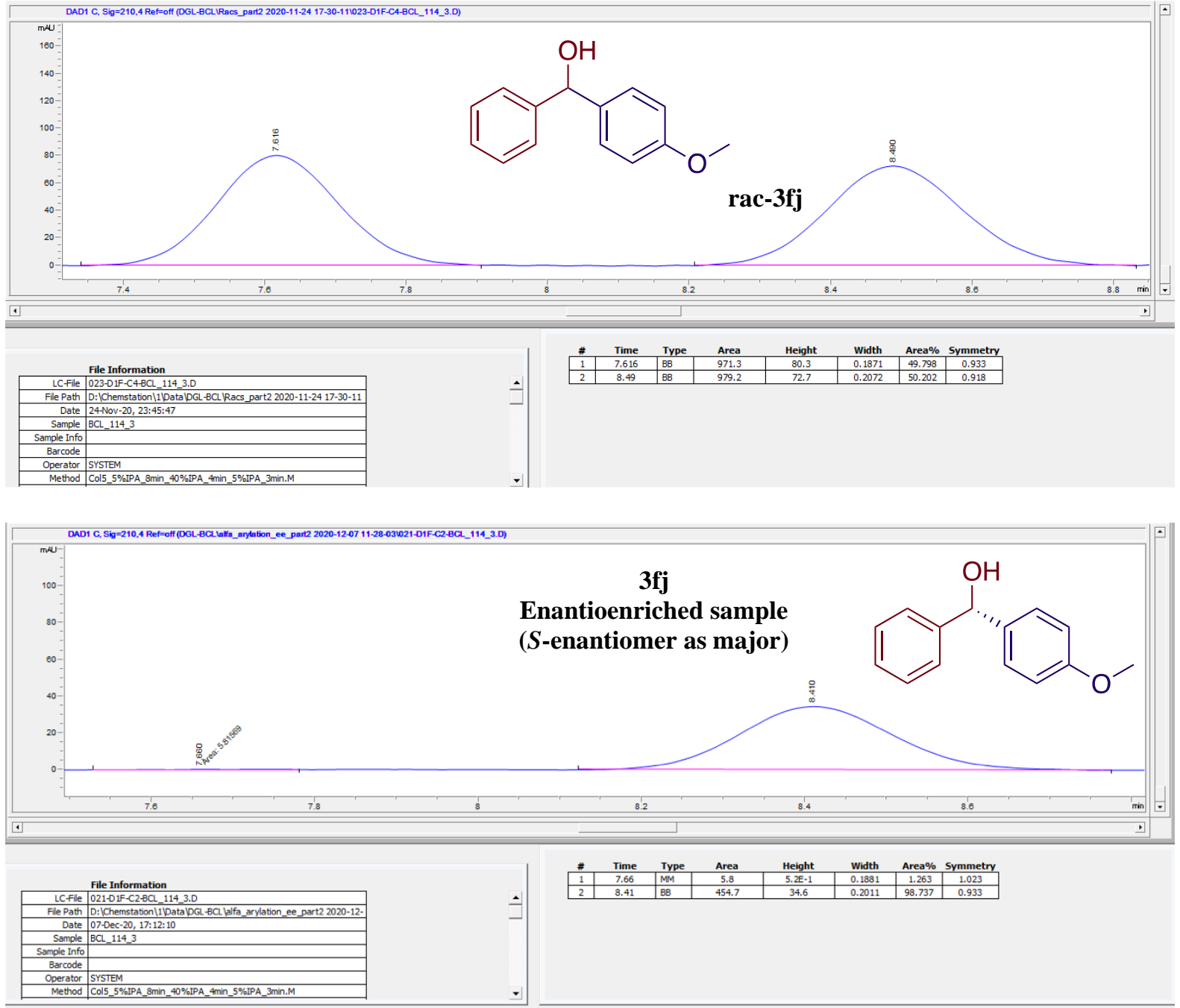
3fk
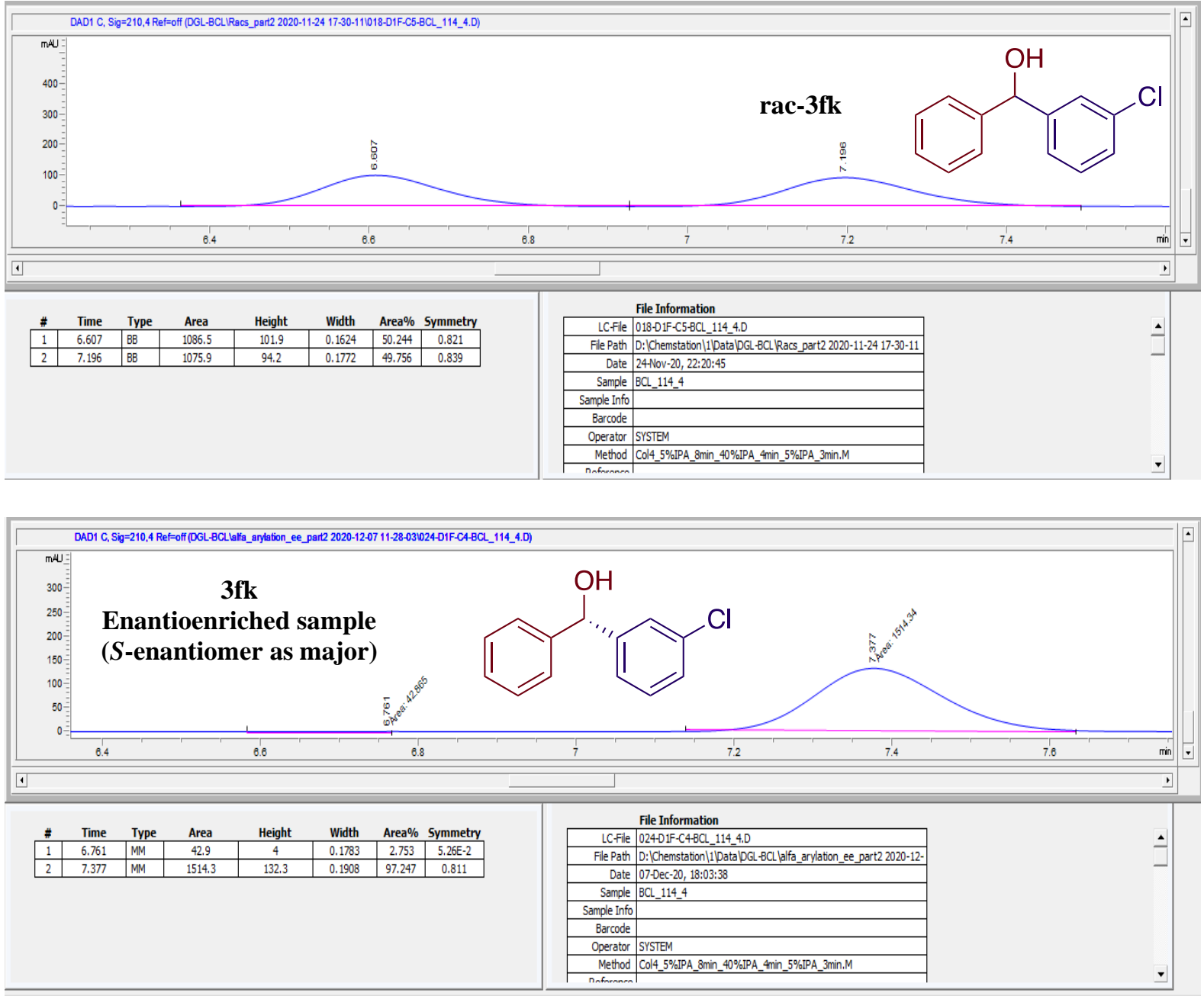
3sa
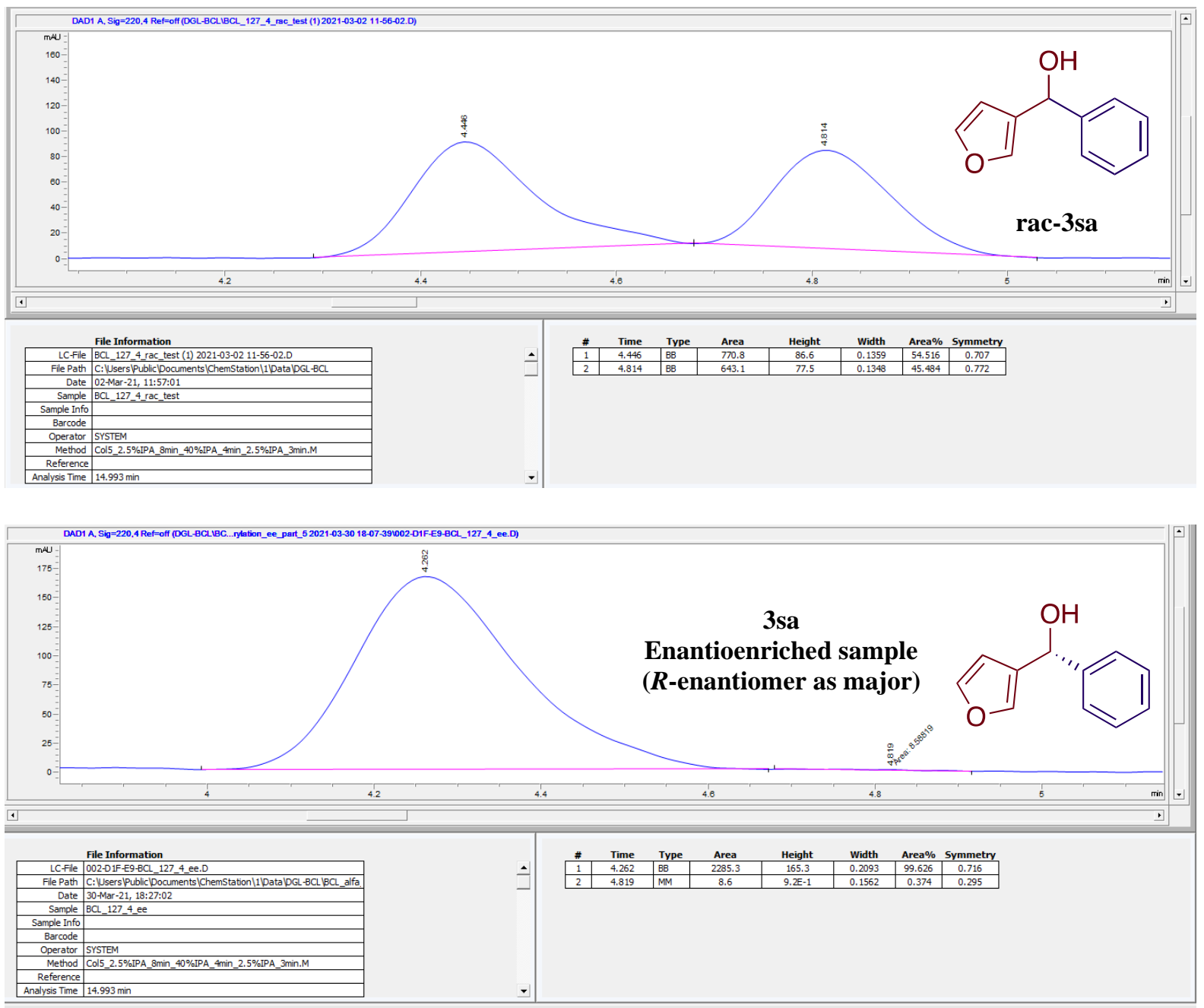
$3 q a$
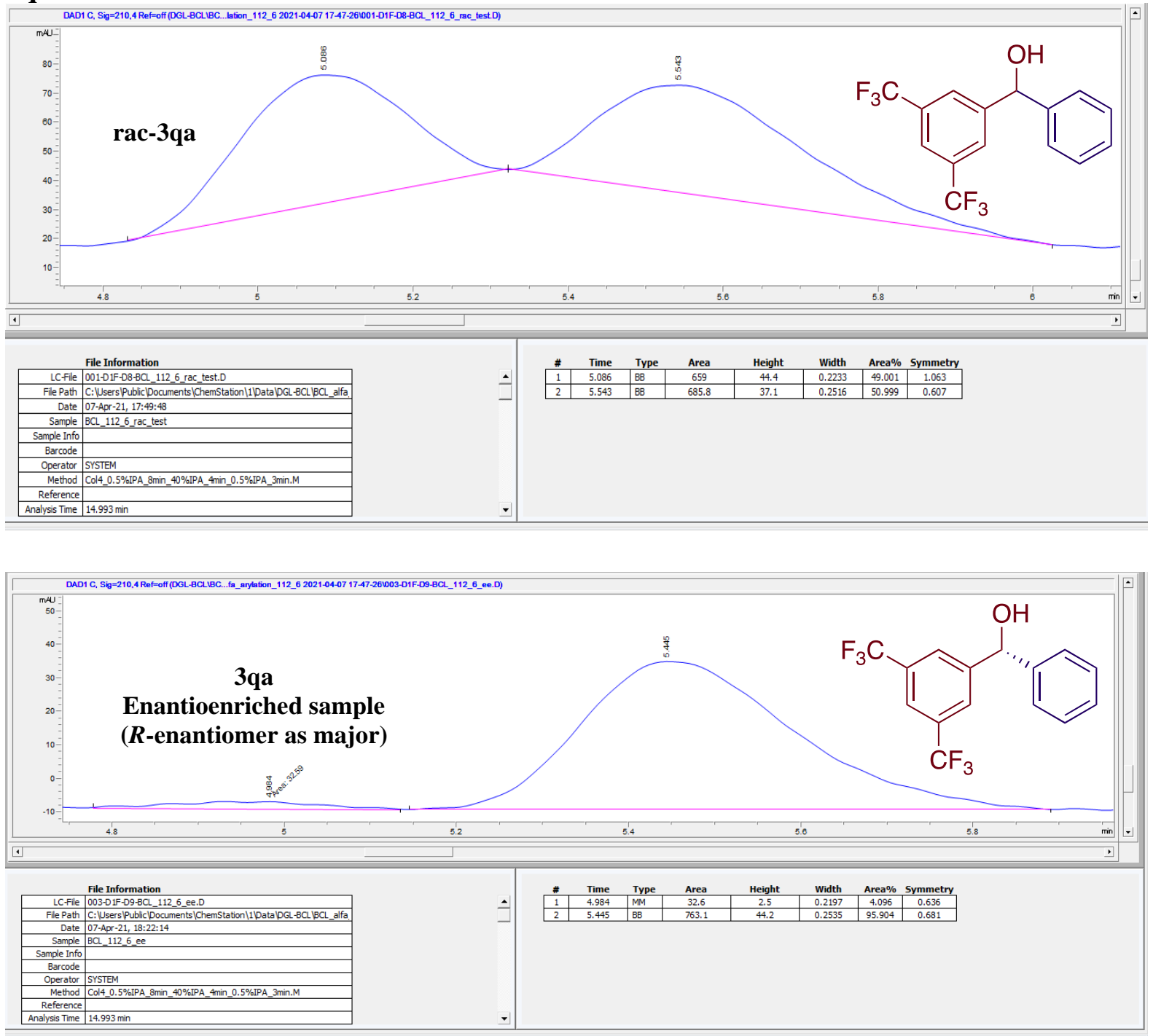


\section{HRMS reports}
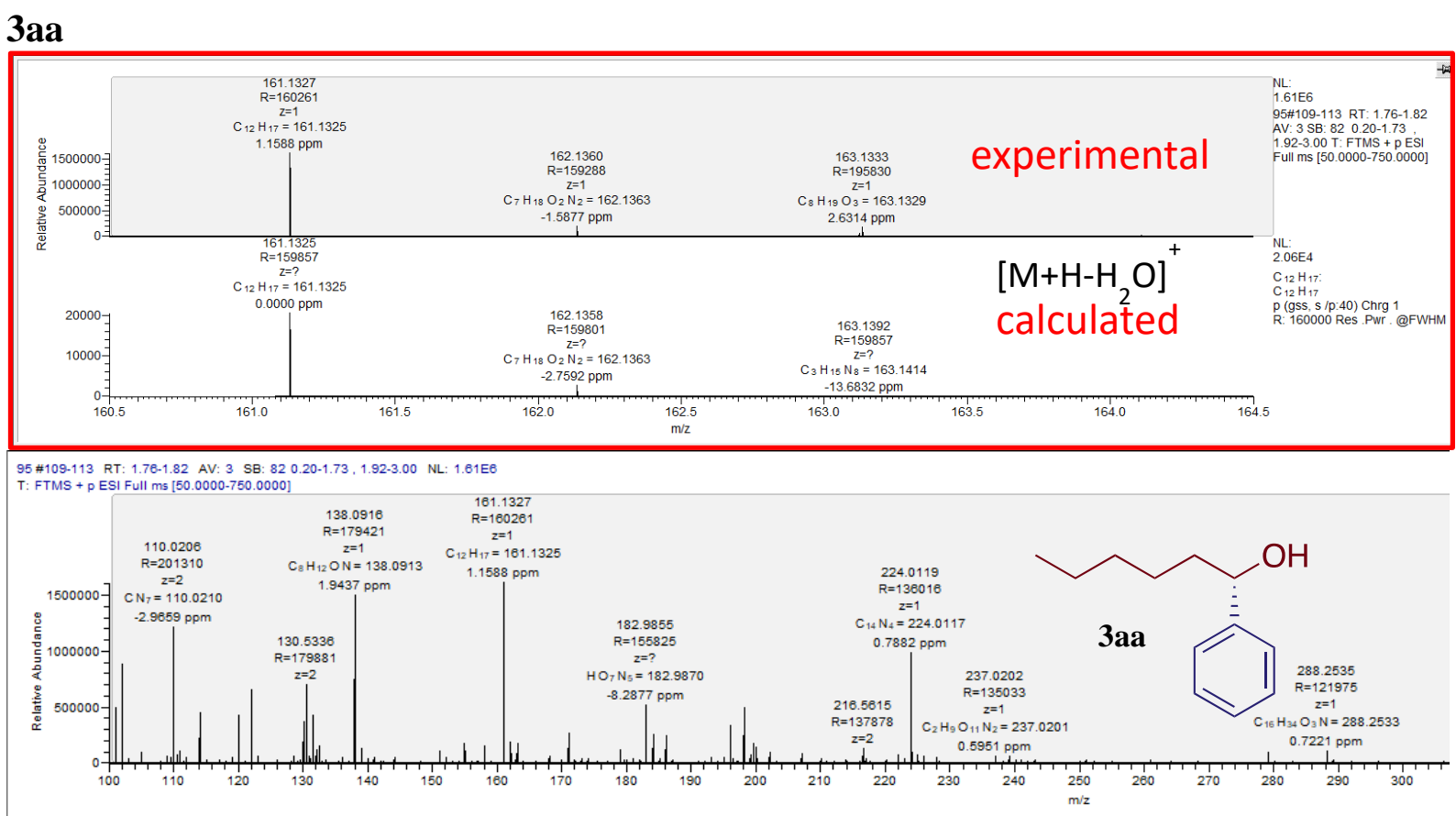

3ab
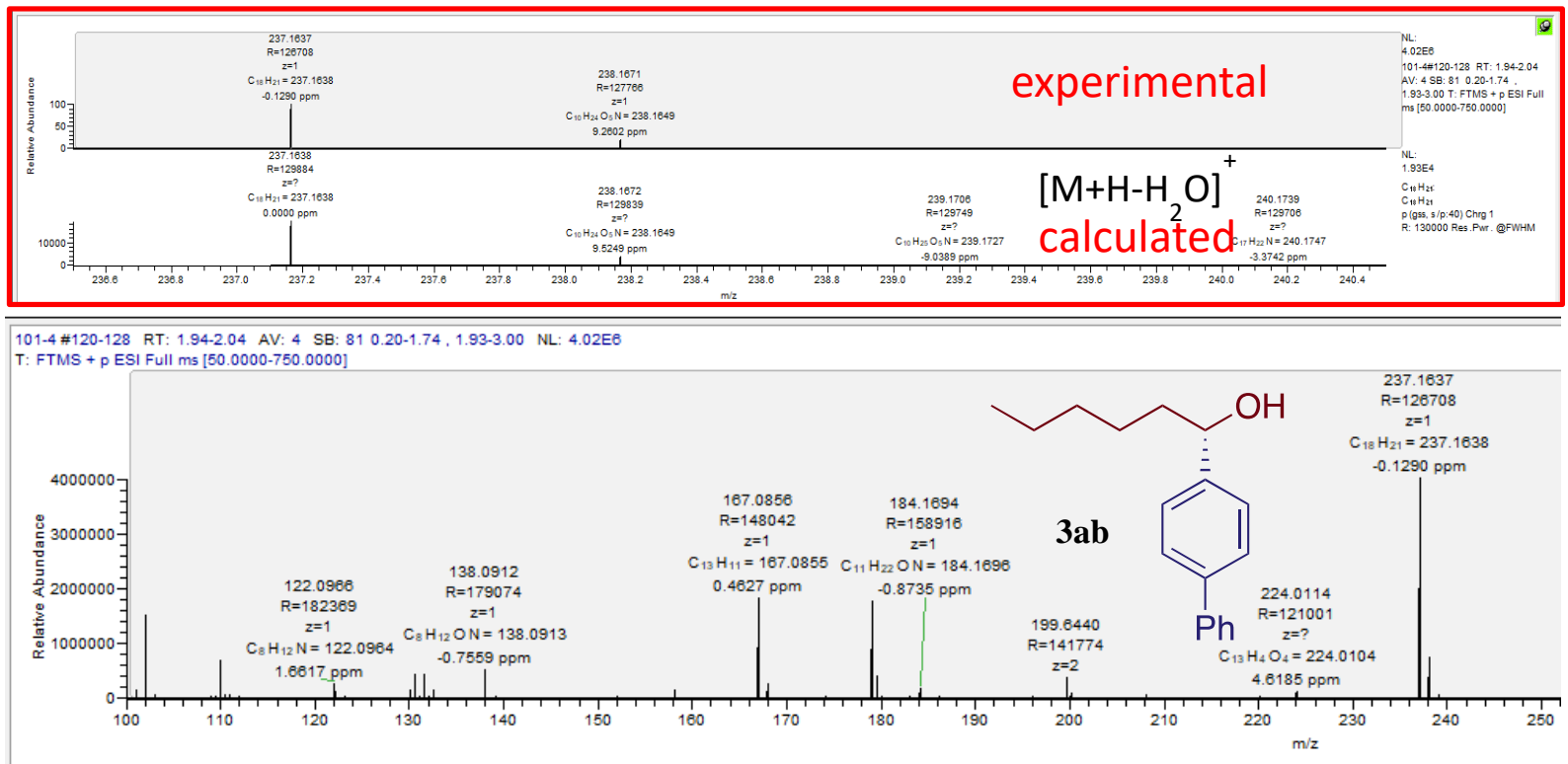
3ac

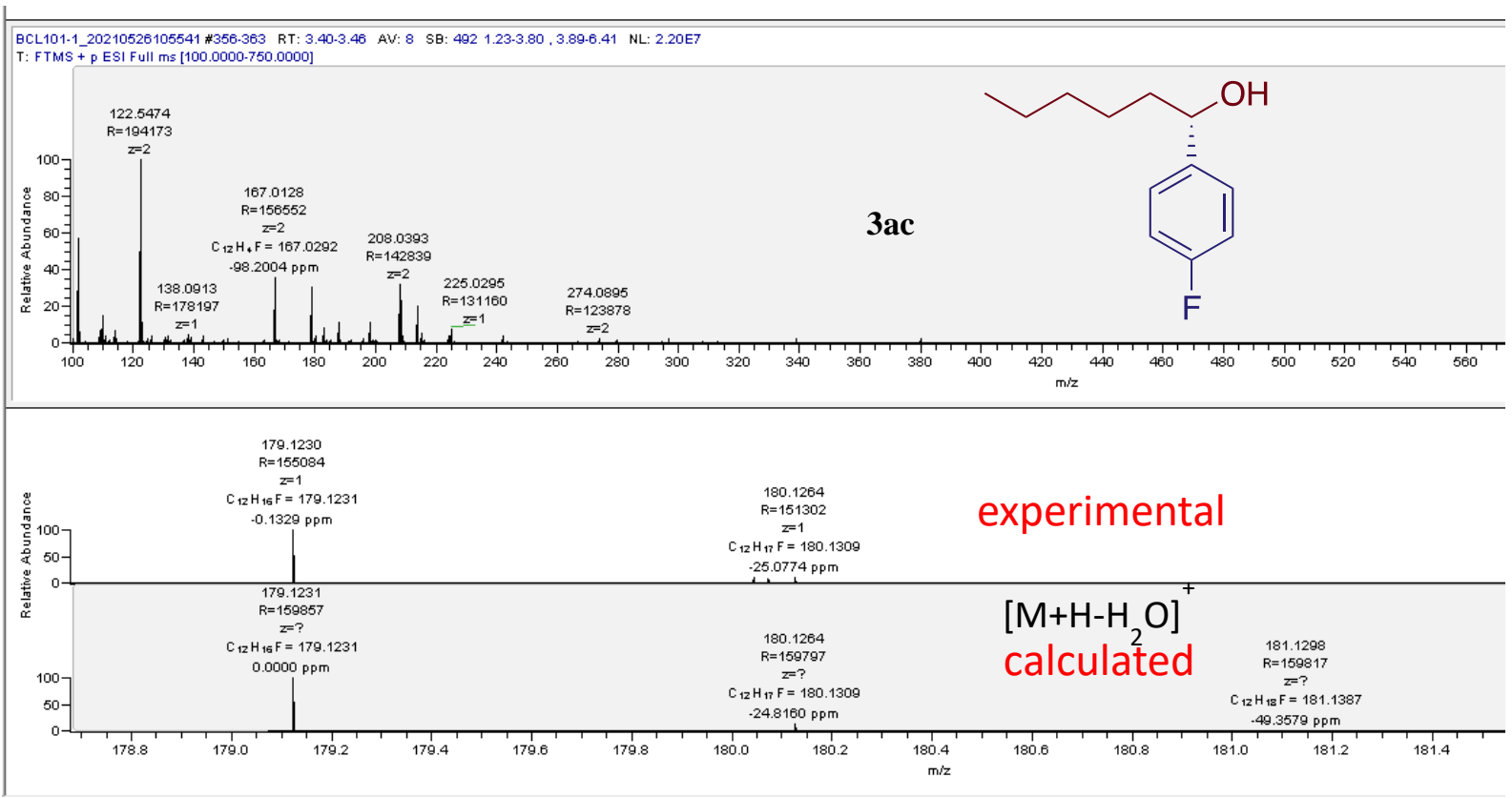

3ae
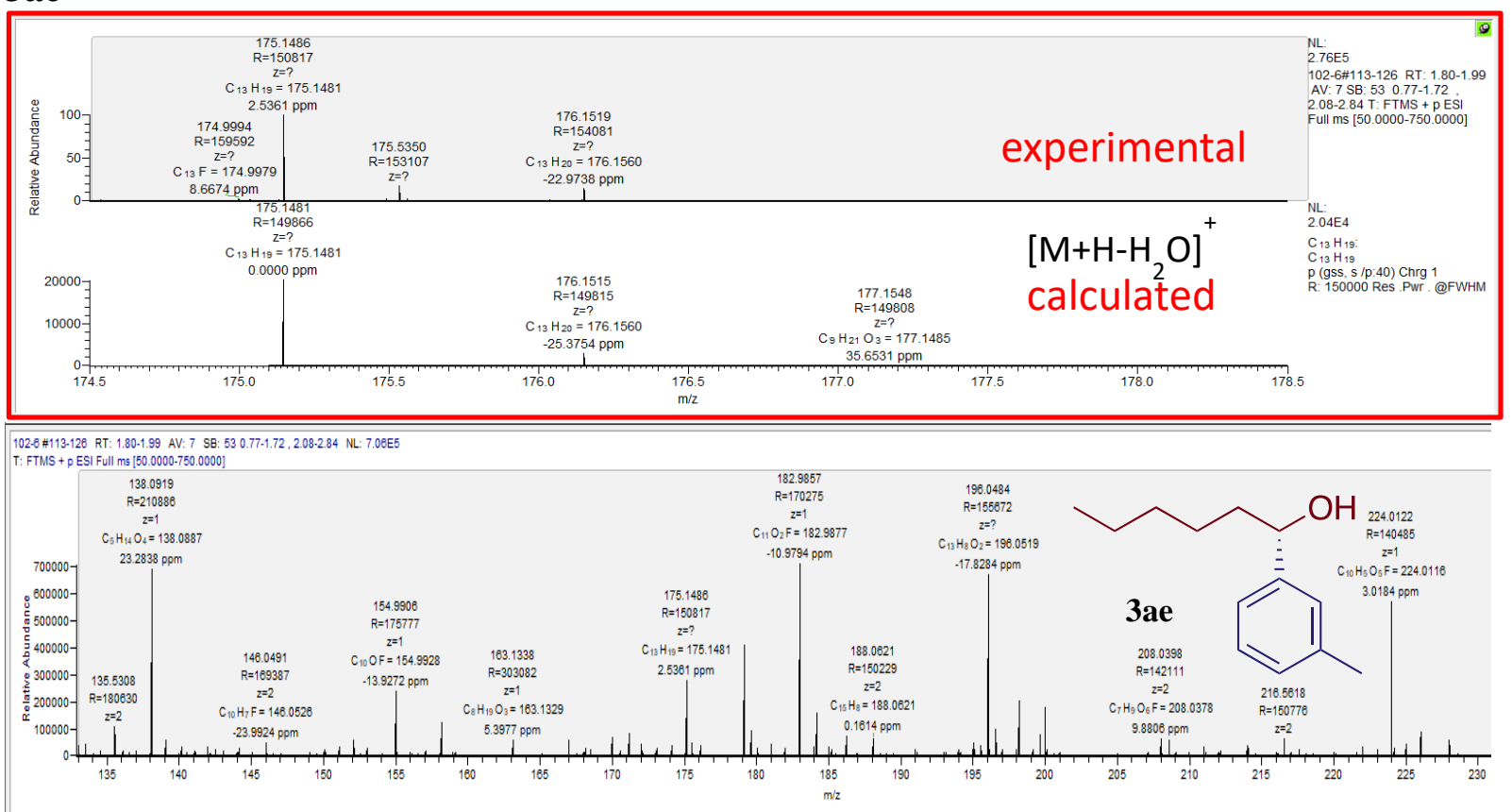
3ag
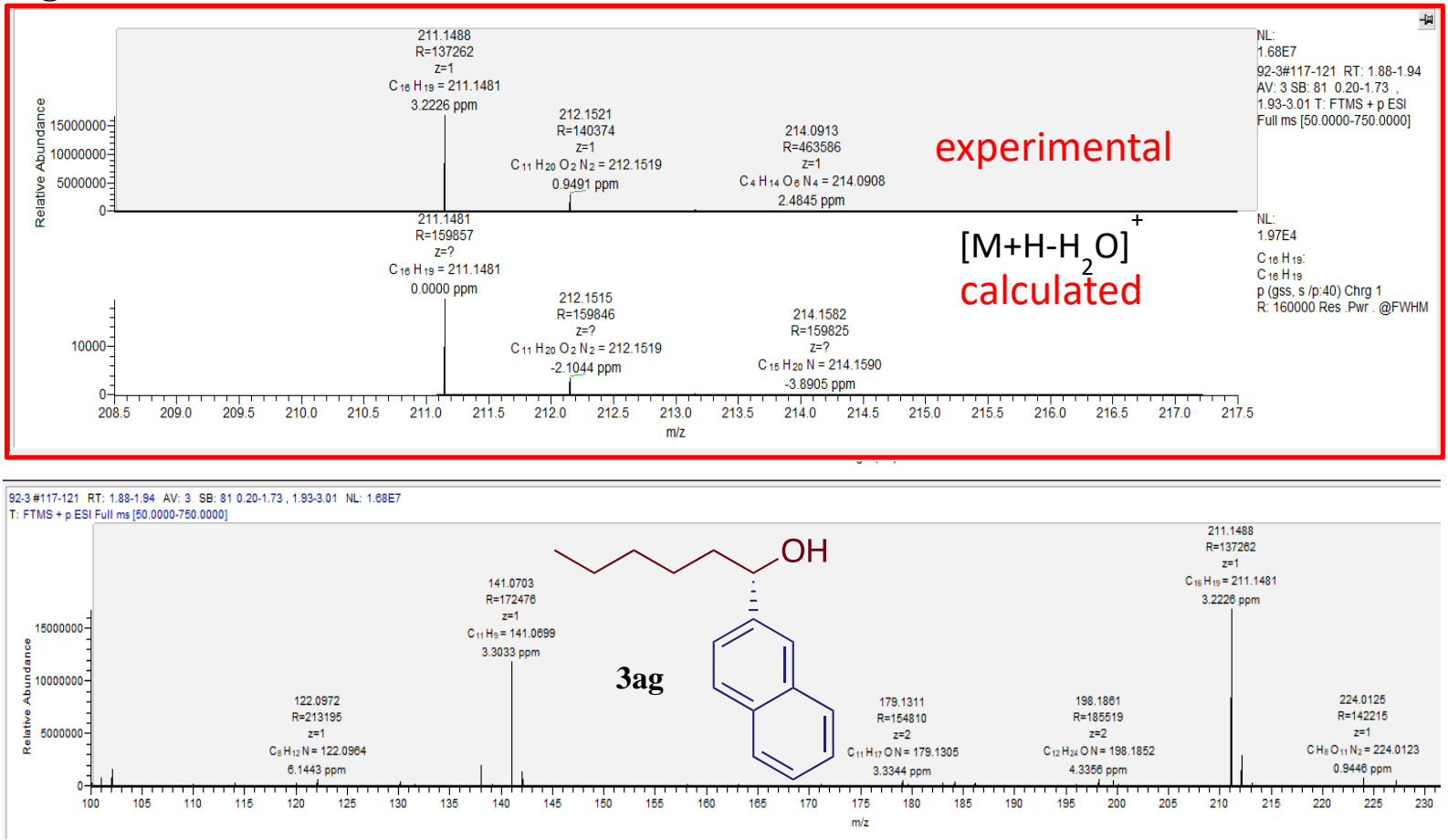

\section{3ah}
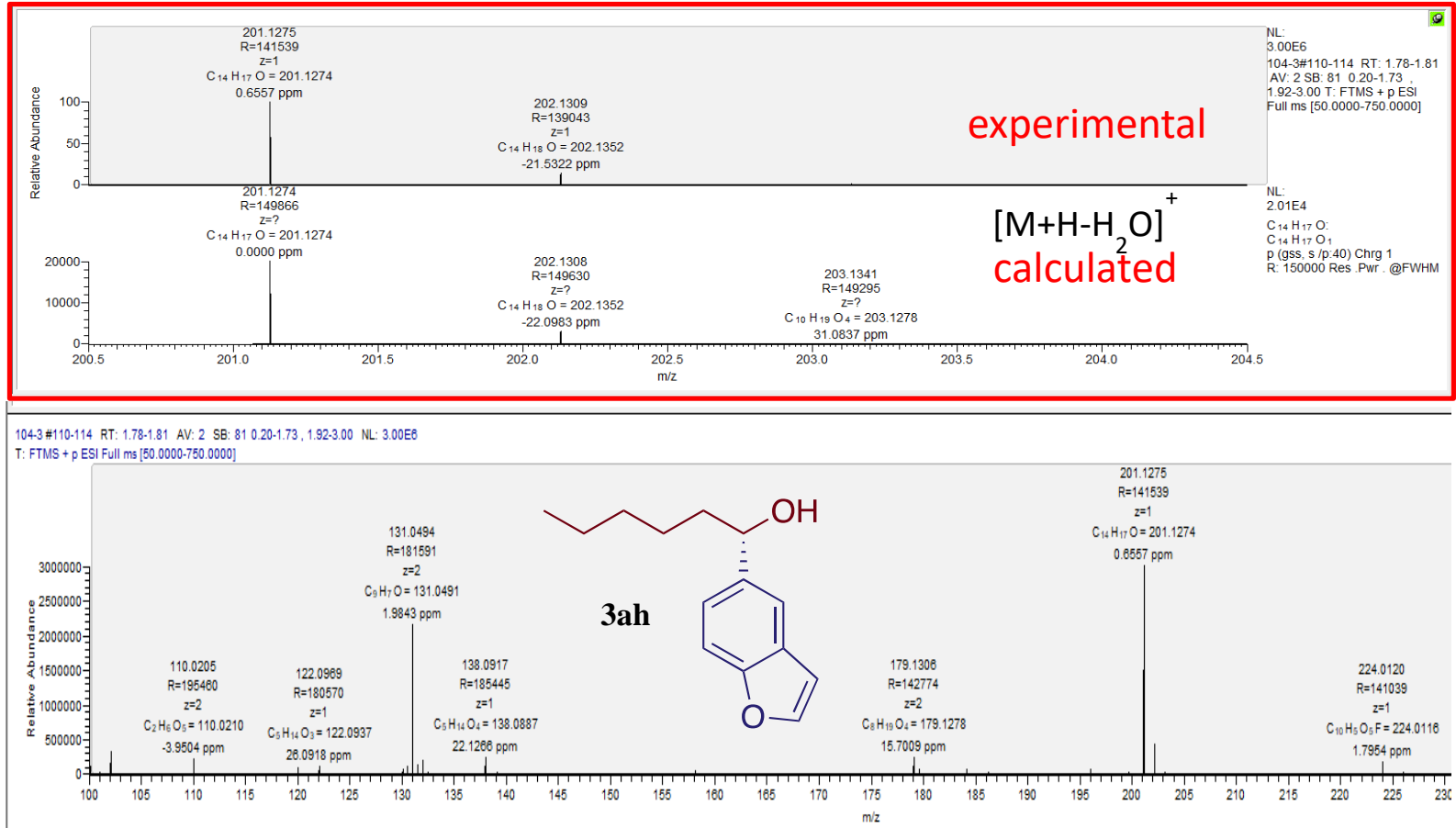
3ai

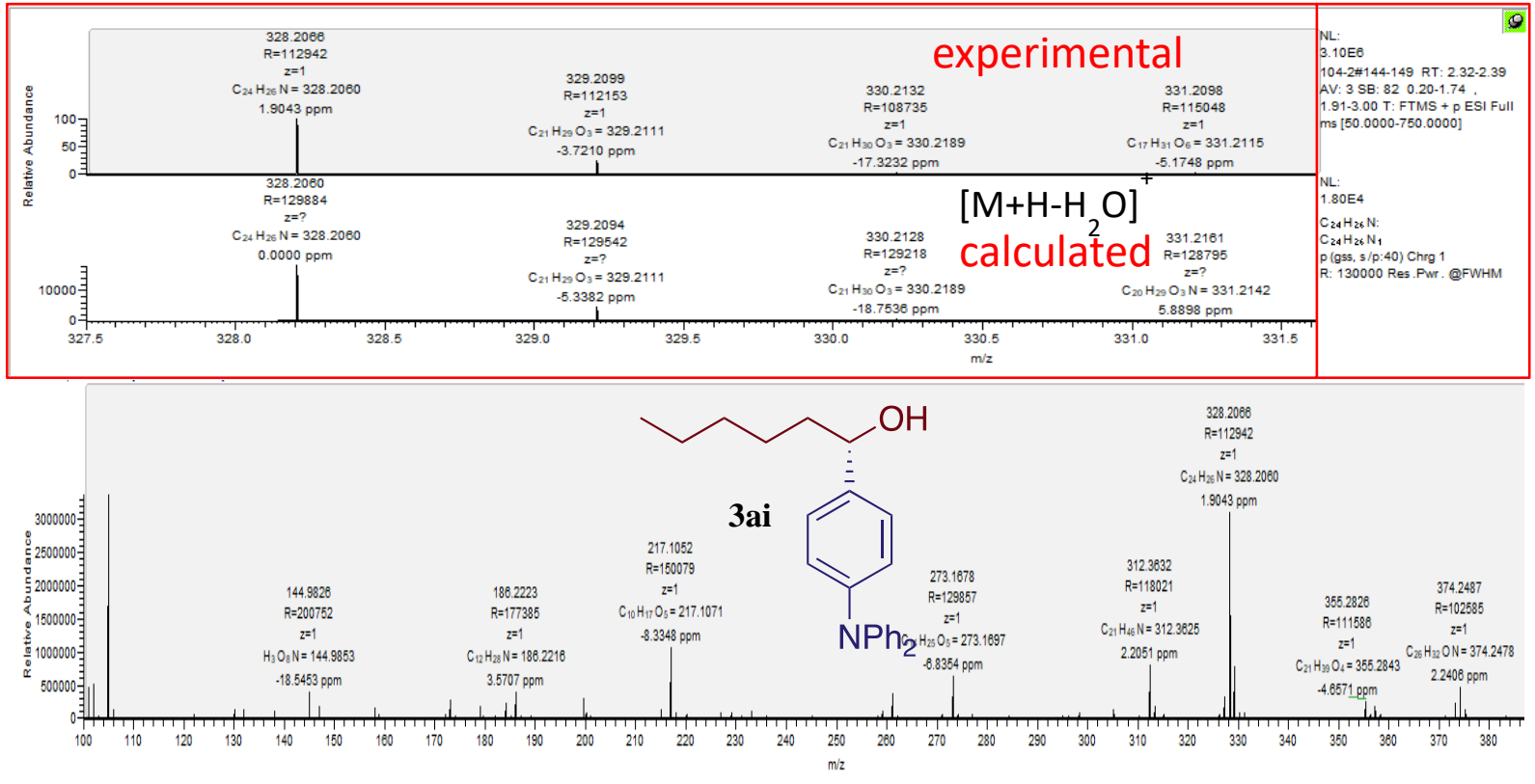

3ga
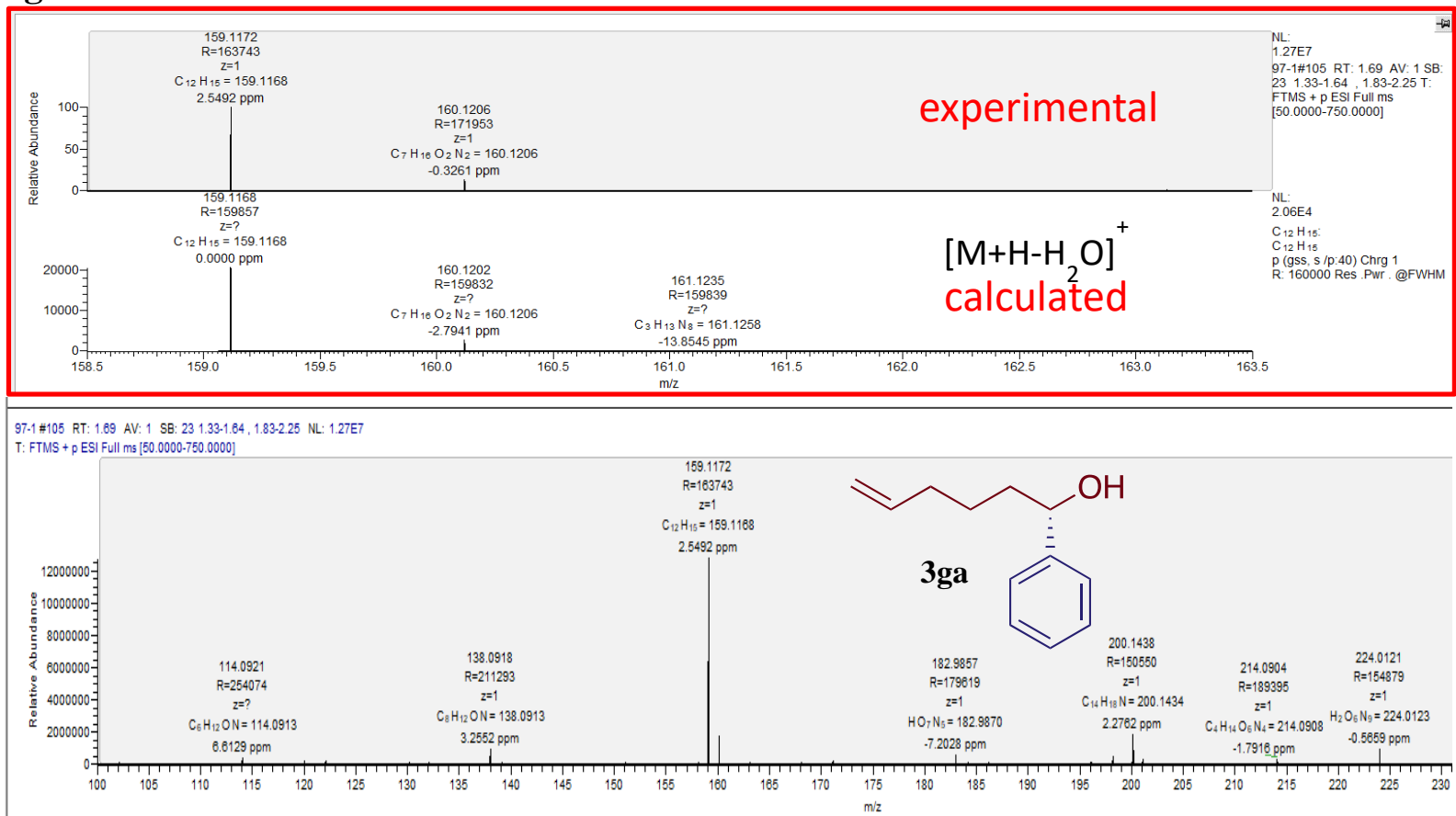


\section{3ha}

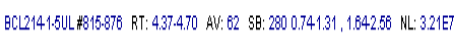

T: FTMS + p ESI Full ms 1000.0000 .750 .00000

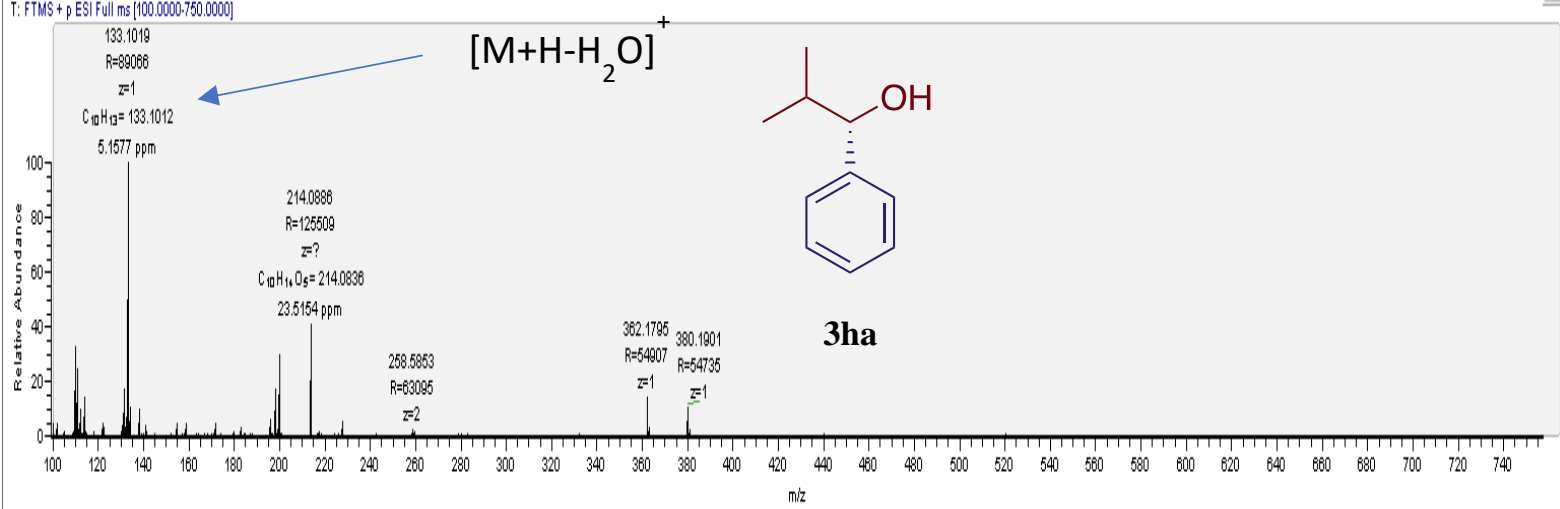

3ia

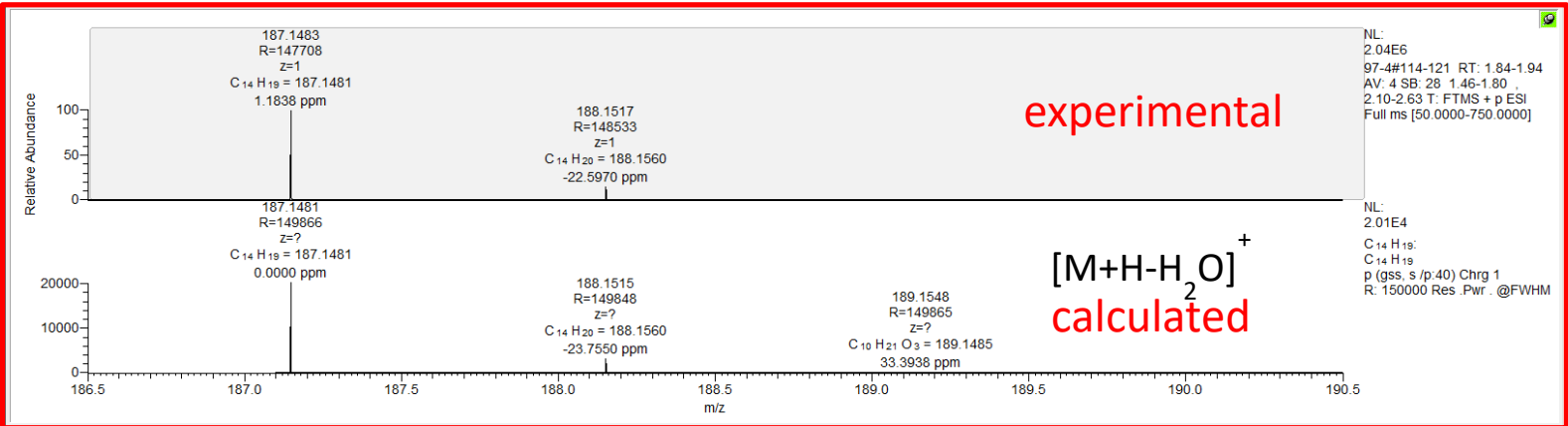

97.4\#114-121 RT: 1.84-1.94 AV: 4 SB: 28 1.48-1.80, 2.10-2.63 NL: 2.04E

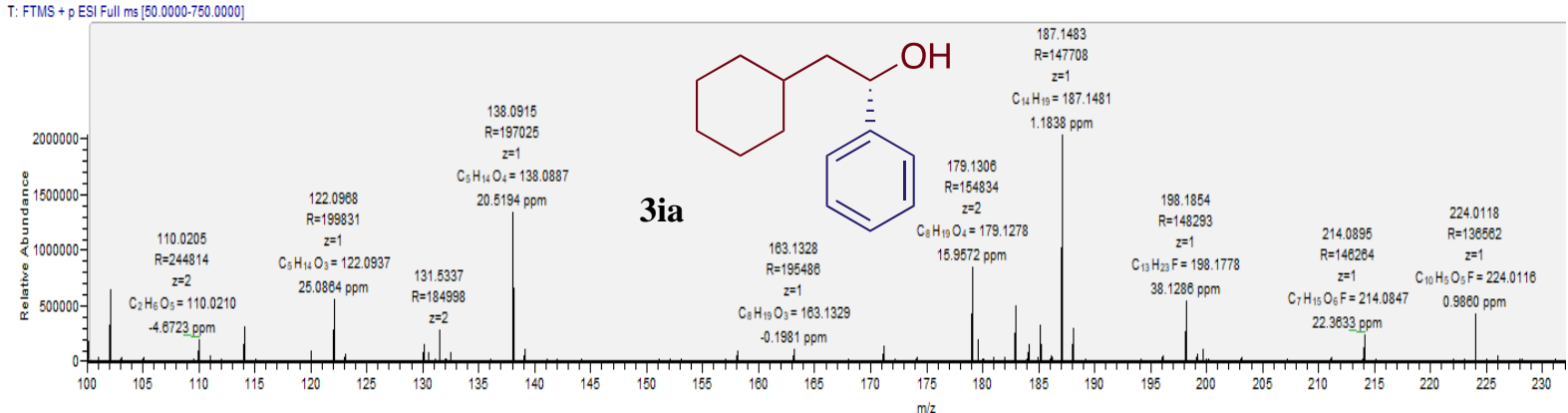



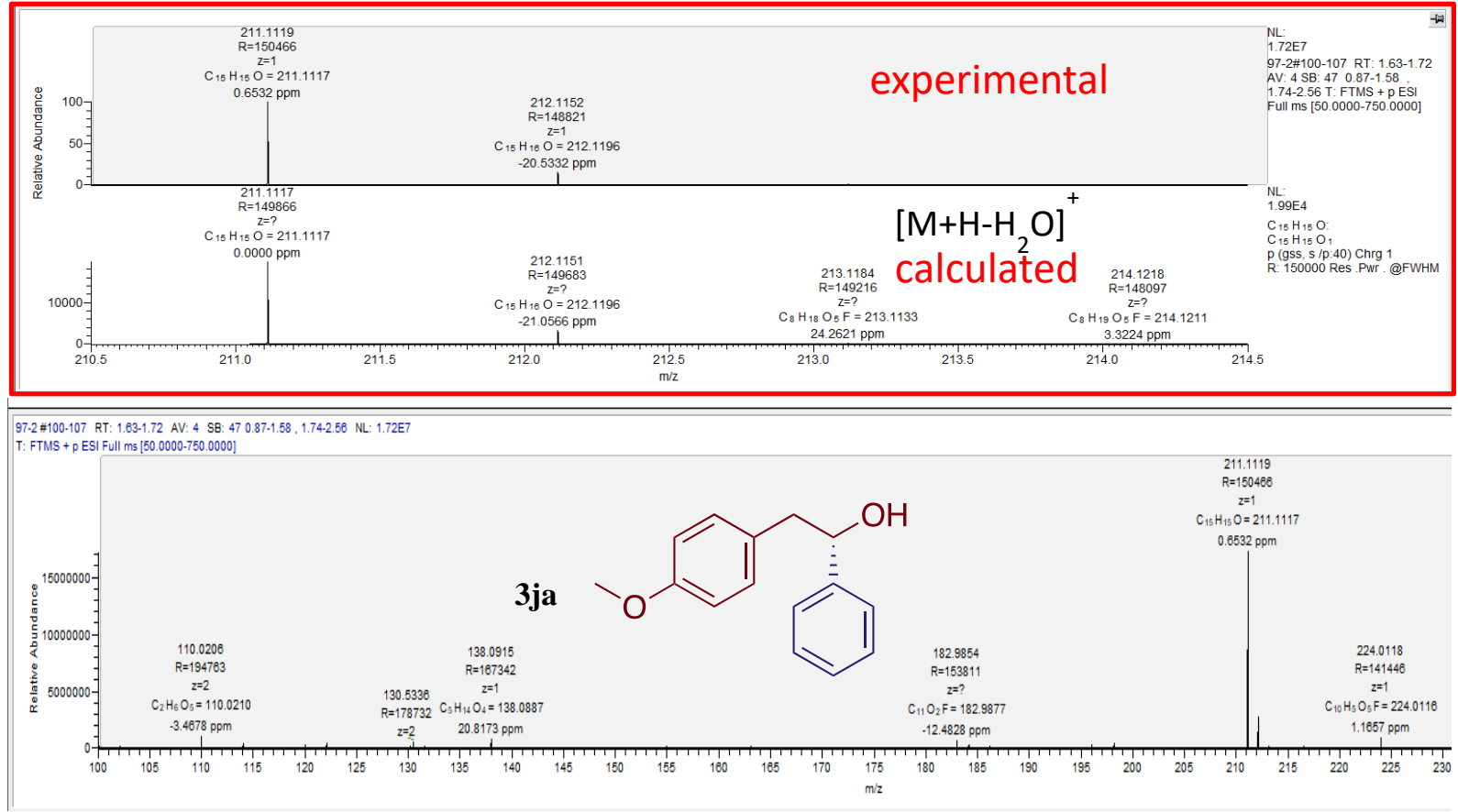

3ka
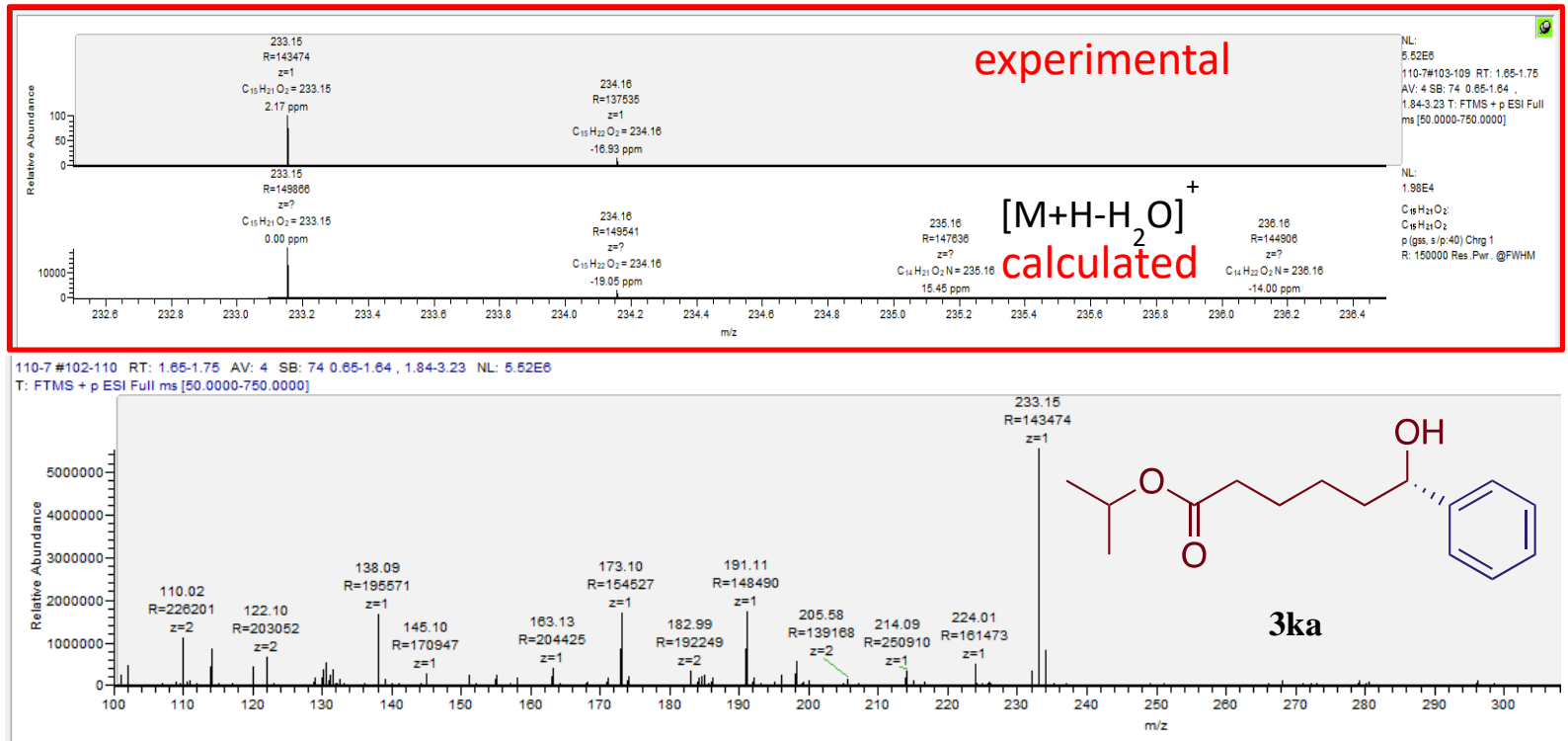
3la
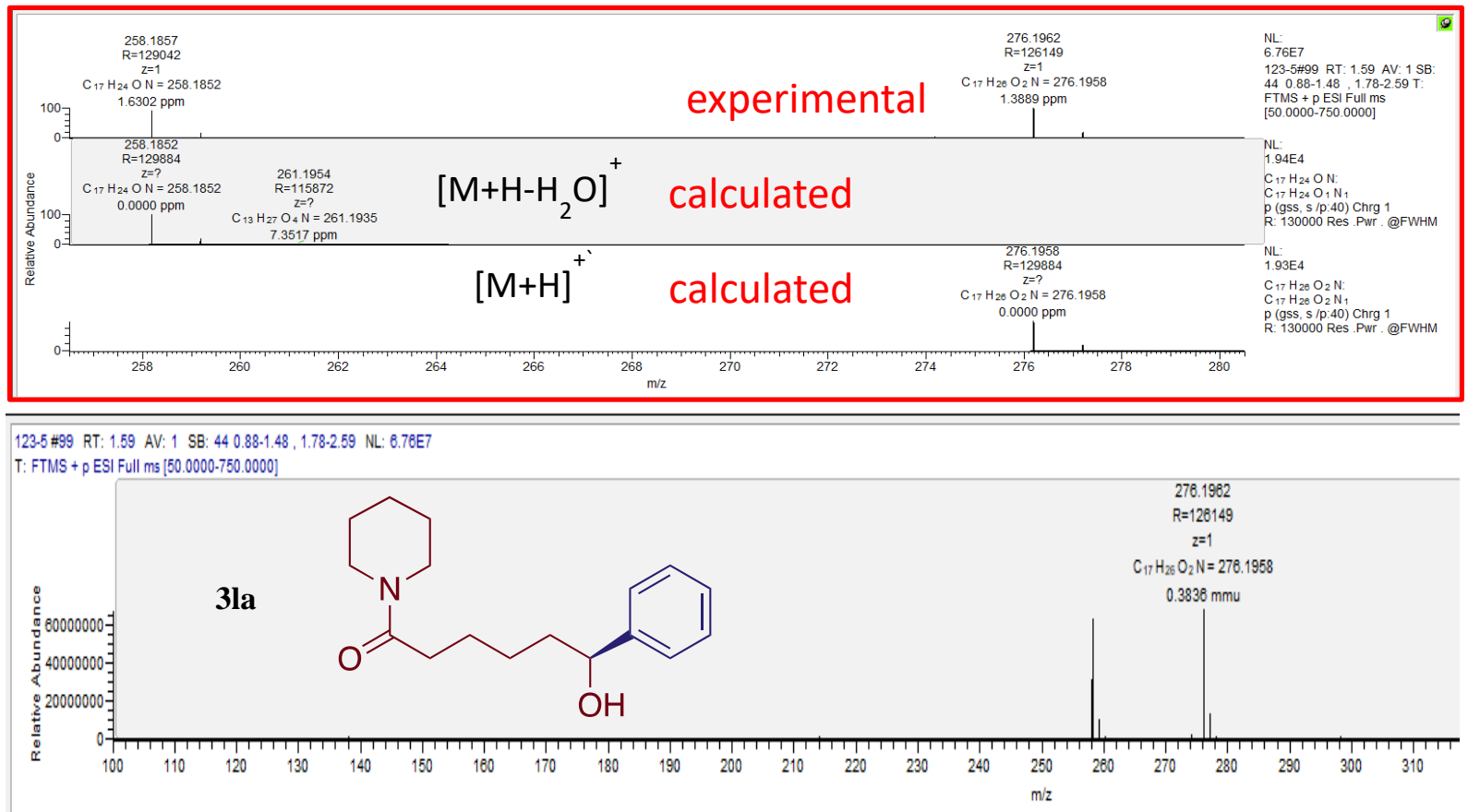

\section{3ma}
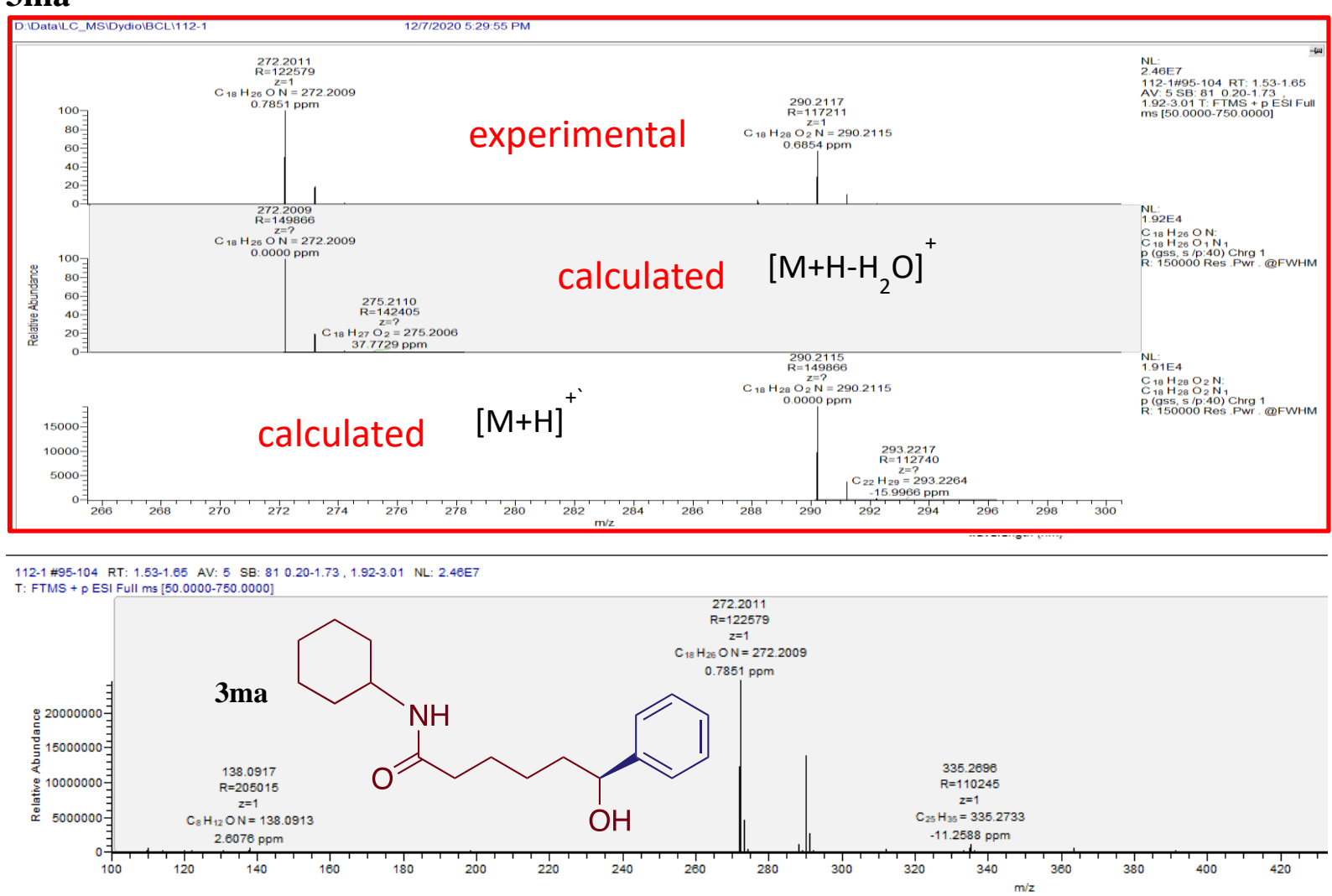
3na
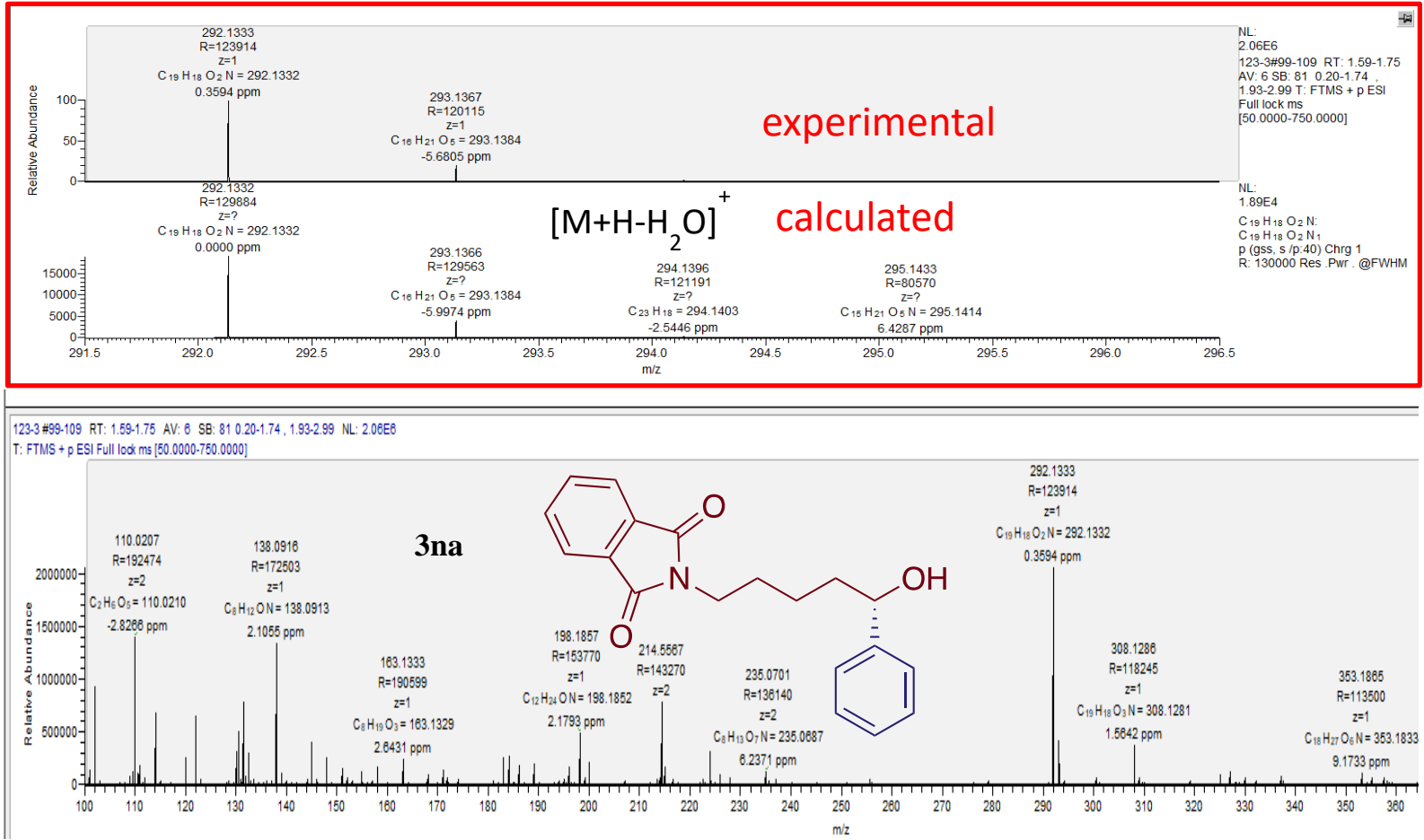

$30 a$
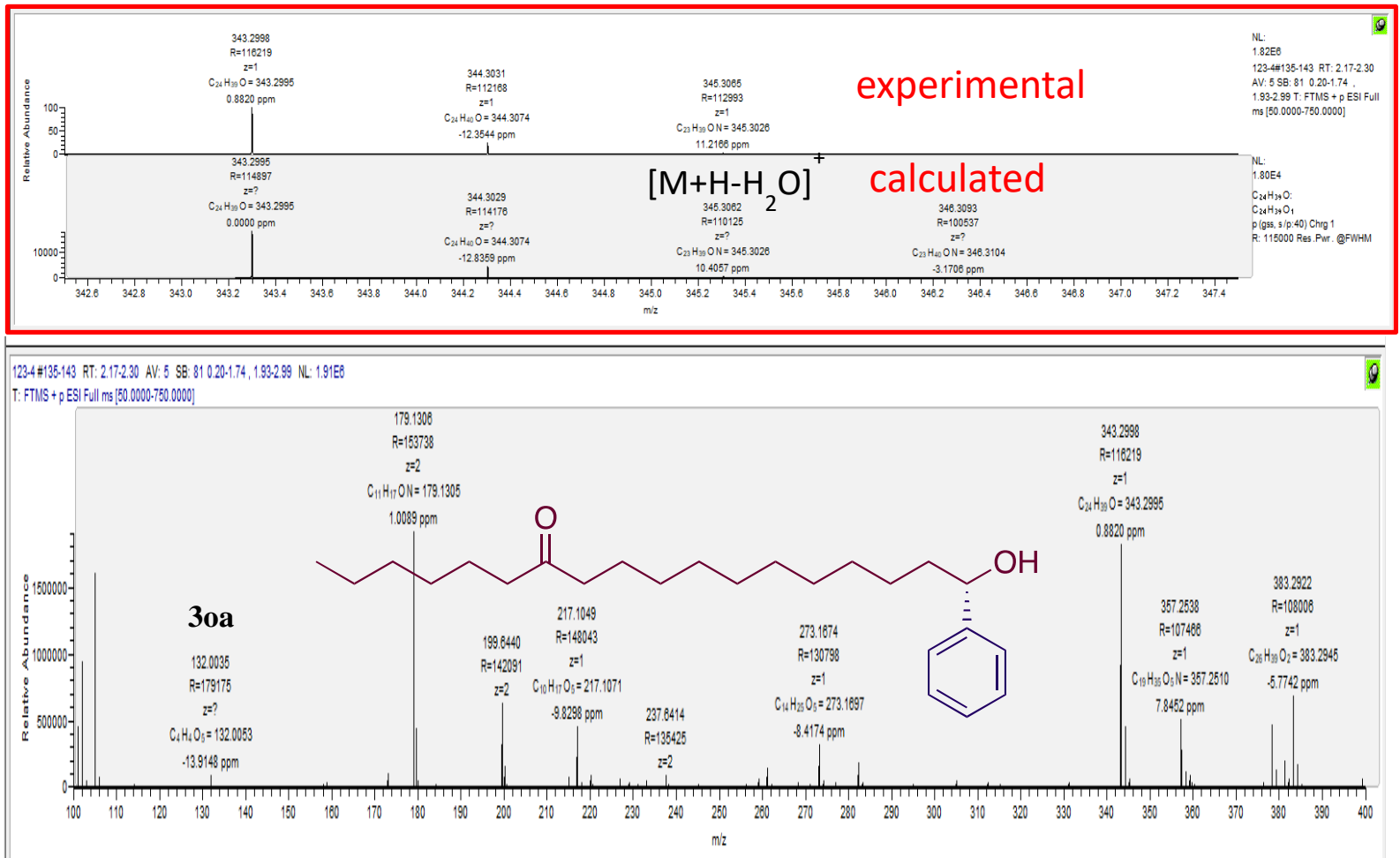

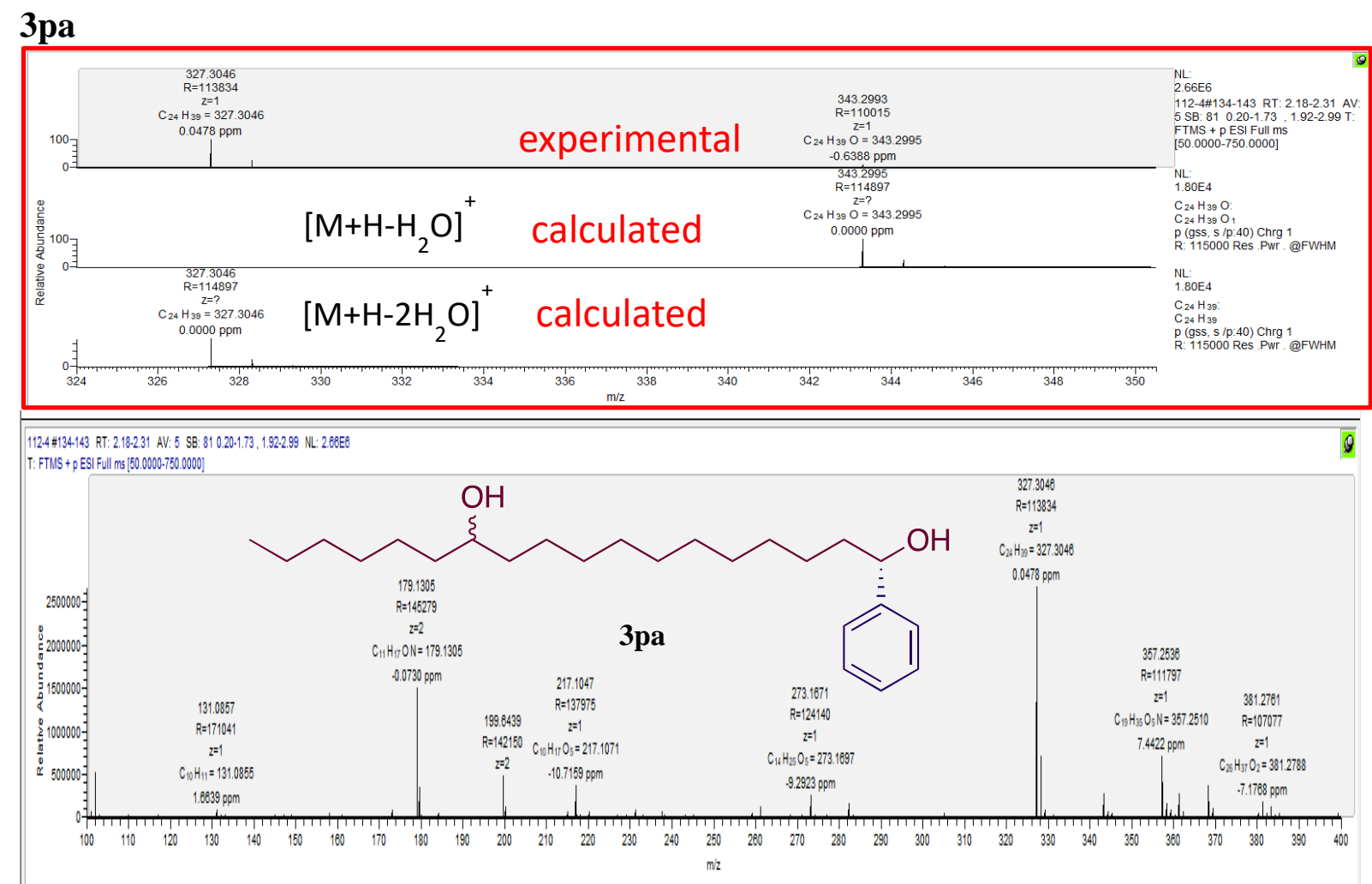

\section{3fj}

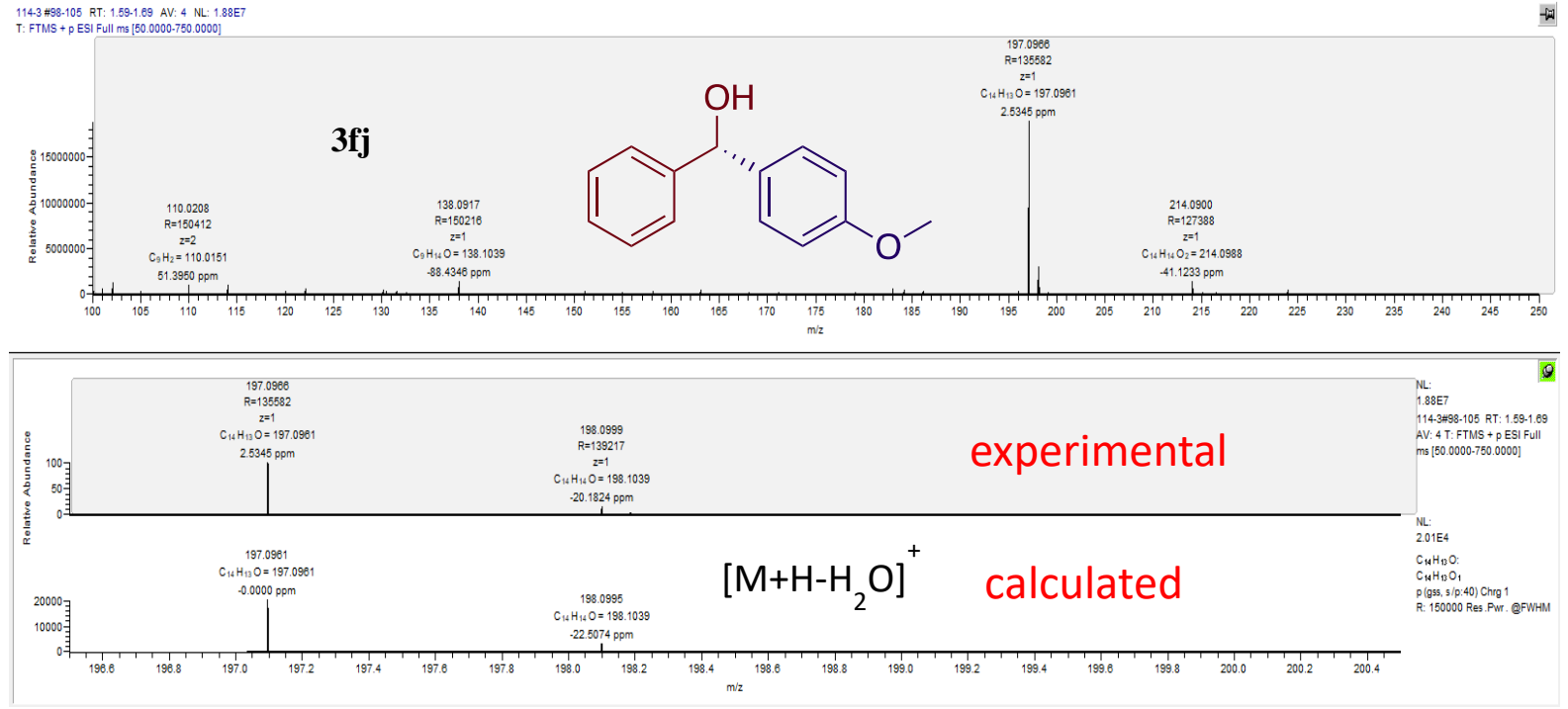




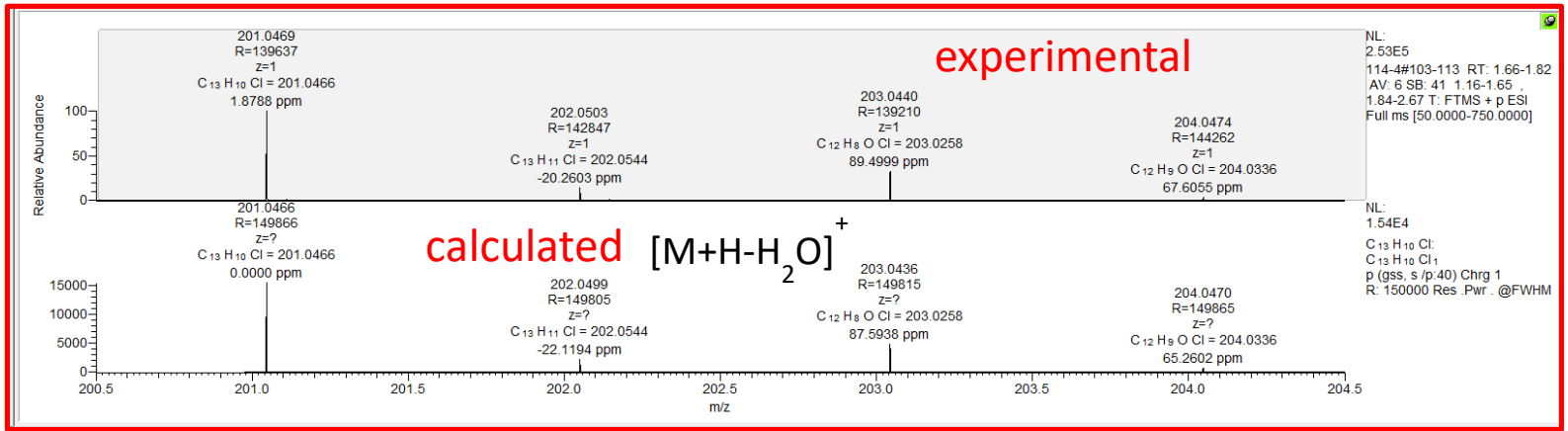

114-4 \#103-113 RT: 1.88-1.82 AV: 8 SB: 41 1.18-1.85, 1.84-2.87 NL: 9.10E5

T: FTMS + p ESI Full ms [50.0000.750.0000]

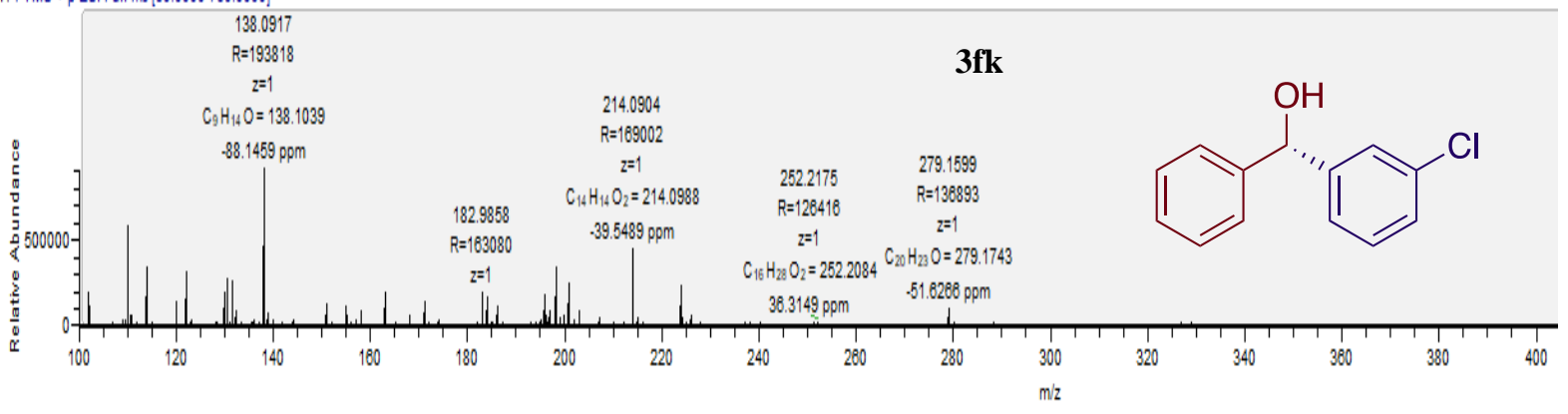

\section{3qa}

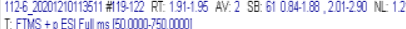
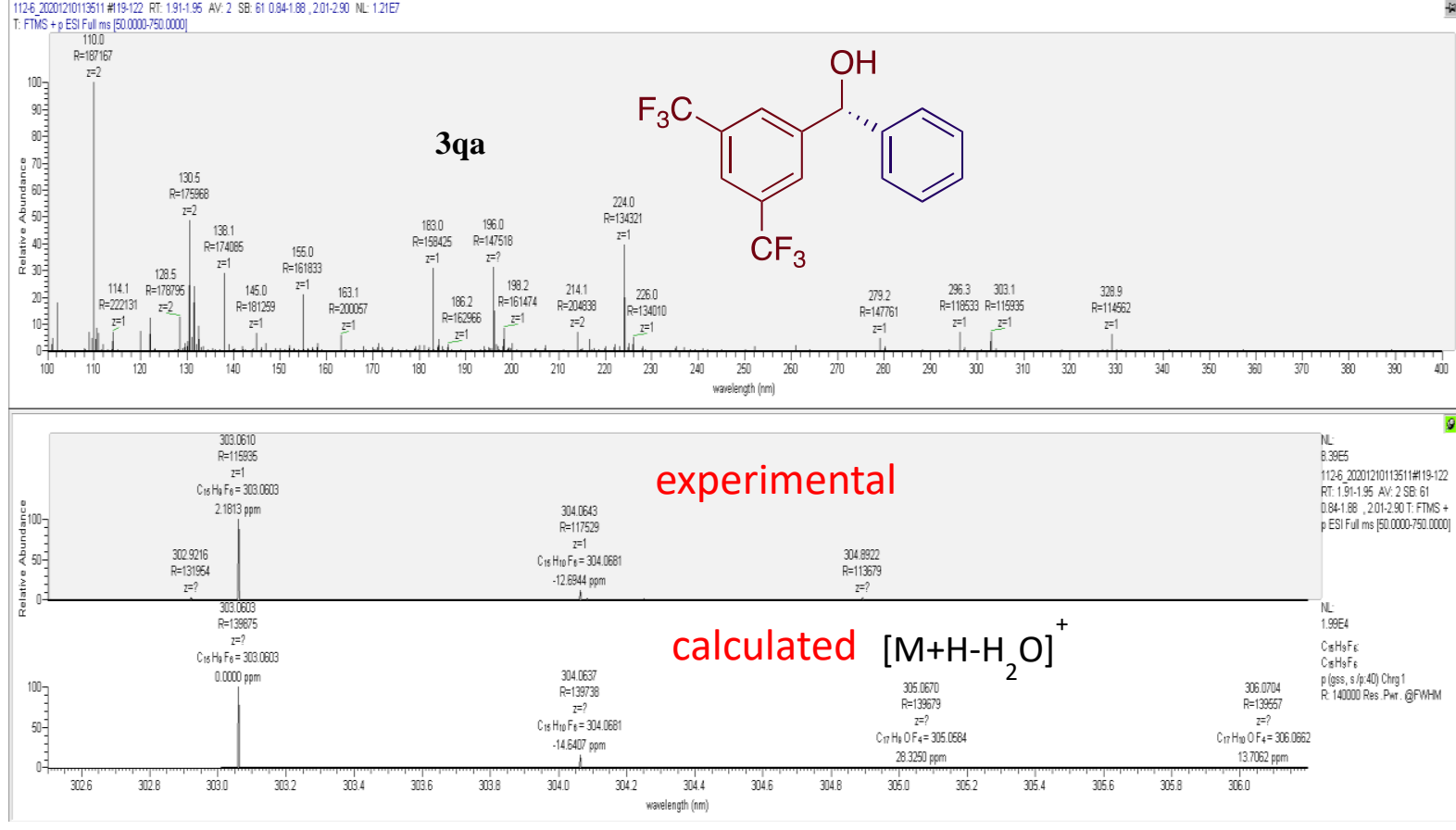
3ra

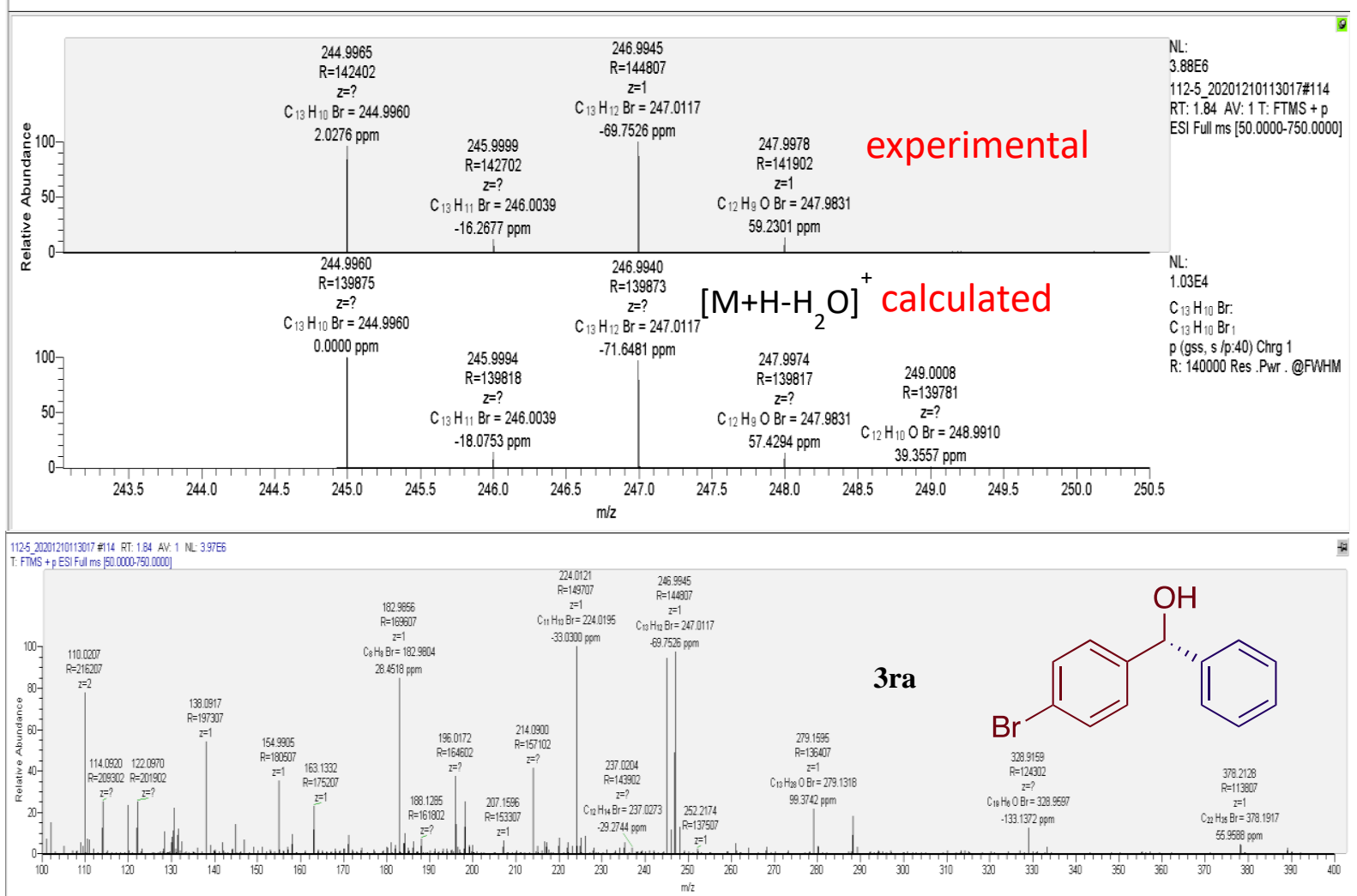

3sa

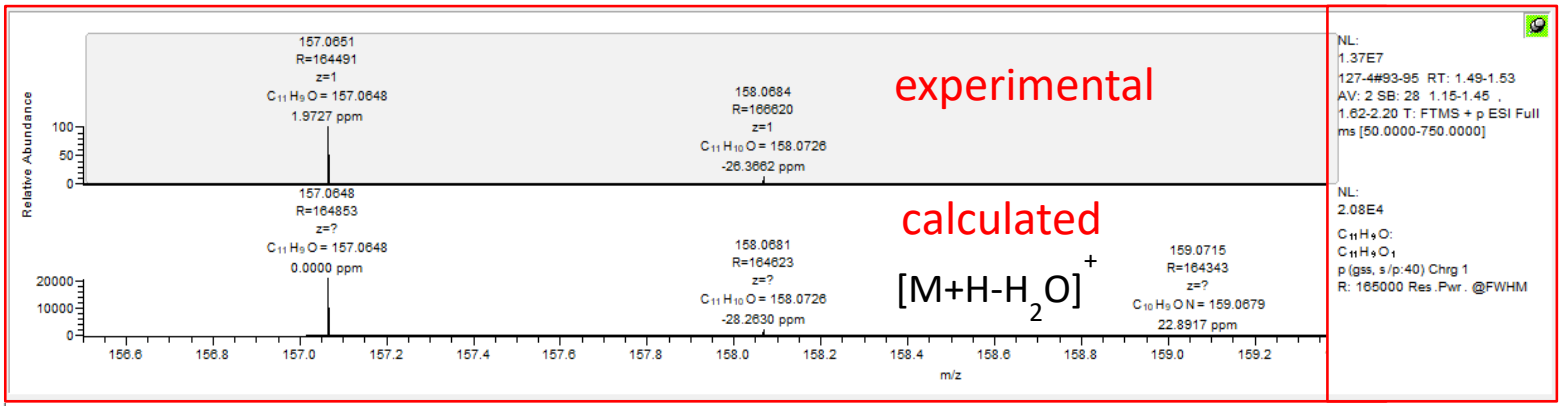

127.4 499.96 RT: 1.48-1.53 AV: 2 SB: 28 1.15-1.45, 1.62.2.20 NL: 1.37E7

T. FTMS + p PSF Ful ma [00.0000-750.0000]

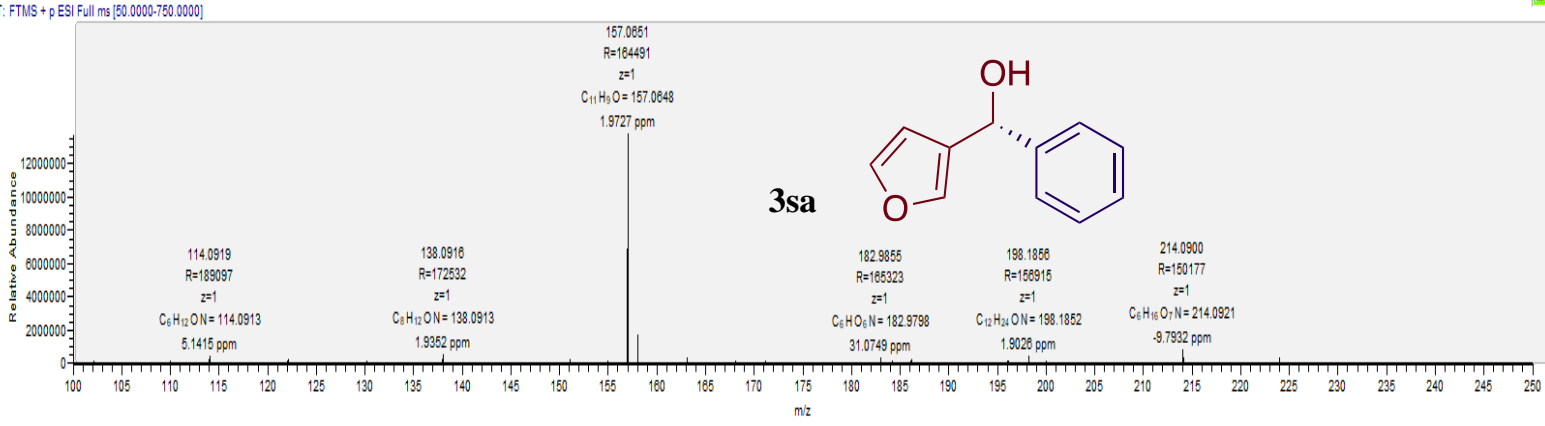

